\title{
Realisation functors in tilting theory
}

\author{
Chrysostomos Psaroudakis $^{1}$. Jorge Vitória ${ }^{2}$
}

Received: 12 December 2016 / Accepted: 9 May 2017 / Published online: 17 August 2017

(C) The Author(s) 2017. This article is an open access publication

\begin{abstract}
Derived equivalences and t-structures are closely related. We use realisation functors associated to t-structures in triangulated categories to establish a derived Morita theory for abelian categories with a projective generator or an injective cogenerator. For this purpose we develop a theory of (non-compact, or large) tilting and cotilting objects that generalises the preceding notions in the literature. Within the scope of derived Morita theory for rings we show that, under some assumptions, the realisation functor is a derived tensor product. This fact allows us to approach a problem by Rickard on the shape of derived equivalences. Finally, we apply the techniques of this new derived Morita theory to show that a recollement of derived categories is a derived version of a recollement of abelian categories if and only if there are tilting or cotilting t-structures glueing to a tilting or a cotilting t-structure. As a further application, we answer a question by $\mathrm{Xi}$ on a standard form for recollements of derived module categories for finite dimensional hereditary algebras.
\end{abstract}

Keywords t-Structure $\cdot$ Silting $\cdot$ Tilting $\cdot$ Cosilting $\cdot$ Cotilting $\cdot$ Recollement $\cdot$ Derived equivalence $\cdot$ Realisation functor $\cdot$ Homological embedding

The first named author was supported by the Norwegian Research Council (NFR 221893) under the project Triangulated categories in Algebra at the Norwegian University of Science and Technology (NTNU). The second named author was initially supported by the German Research Council through the SFB 701 at the University of Bielefeld, then by a Marie Curie Intra-European Fellowship within the 7th European Community Framework Programme (PIEF-GA-2012-327376) at the University of Verona and, in the final stages of this project, by the Engineering and Physical Sciences Research Council of the United Kingdom, Grant number EP/N016505/1 at City, University of London.

$凶$ Jorge Vitória

jorge.vitoria@city.ac.uk

Chrysostomos Psaroudakis

chrysostomos.psaroudakis@mathematik.uni-stuttgart.de

1 Institute of Algebra and Number Theory, University of Stuttgart, Pfaffenwaldring 57, 70569 Stuttgart, Germany

2 Department of Mathematics, City, University of London, Northampton Square, London EC1V 0HB, UK 
Mathematics Subject Classification $18 \mathrm{E} 30 \cdot 18 \mathrm{E} 35 \cdot 16 \mathrm{E} 30 \cdot 16 \mathrm{E} 35 \cdot 14 \mathrm{~F} 05 \cdot 16 \mathrm{G} 20$

\section{Contents}

1 Introduction . . . . . . . . . . . . . . . . . . . . . . . 966

2 Preliminaries: t-structures, recollements and glueing . . . . . . . . . . . . . . . . . . . . . 969

3 Realisation functors . . . . . . . . . . . . . . . . . . . . . . . . . . . . 974

4 Silting and cosilting $t$-structures $\ldots \ldots \ldots \ldots \ldots \ldots \ldots$

5 Derived equivalences. . . . . . . . . . . . . . . . . . . . . . . . . . 998

6 Recollements of derived categories _ . . . . . . . . . . . . . . . . . . . 1006

Appendix A: On Beilinson's realization functor (by Ester Cabezuelo Fernández and Olaf M. Schnürer) 1020

References . . . . . . . . . . . . . . . . . . . . . . . . . 1025

\section{Introduction}

\subsection{Motivation}

Derived categories, equivalences between them and the associated derived invariants are central objects of study in modern representation theory and algebraic geometry. In representation theory, the results of Rickard [71] and Keller [47] on a derived Morita theory for rings show that compact tilting complexes guarantee the existence of derived equivalences and viceversa. Some derived equivalences can even be described as a derived tensor product with a complex of bimodules [72]. In algebraic geometry, derived equivalences between coherent sheaves of smooth projective varieties all have a standard form: Fourier-Mukai transforms [66]. In both settings, there is a concern with the existence and the shape of derived equivalences. In this paper we propose a unifying approach to the study of derived equivalences of abelian categories: they should be regarded as realisation functors of certain t-structures. In doing so, we are in particular able to establish a derived Morita theory for abelian categories with a projective generator or an injective cogenerator. This is done in terms of a non-compact or large tilting theory, extending and, in some sense, further clarifying the classical cases mentioned above.

In representation theory, there are some motivating predecessors of the non-compact tilting theory that we develop in this paper: large tilting and cotilting modules over rings $[4,29$, $30,77]$ and large silting complexes $[8,78]$. Such non-compact counterparts of the classical theory were largely motivated by the search of properties that are difficult to obtain in the compact world, namely within the realm of approximation theory. However, contrary to the compact case [37,47,71], these non-compact objects lack a certain derived flavour: their endomorphism rings are not derived equivalent to the original ring; they are usually too big $[12,14,23]$. Here is where our approach to derived equivalences, inspired by that of [77], comes to rescue: instead of considering endomorphism rings, one should consider the hearts of the naturally associated t-structures. The corresponding realisation functors then yield derived equivalences.

\subsection{The main results in context}

A large class of t-structures can be generated from the concept of silting object, which was first defined in [50]. In the bounded derived category of finitely generated modules over a finite dimensional algebra, compact silting objects classify bounded t-structures whose 
heart is a module category [48,51]. Compact silting objects were also considered in abstract triangulated categories $[1,17,40,43]$ and, more recently, non-compact silting objects and their associated $t$-structures were studied in derived module categories $[8,78]$. We introduce a common generalisation of these notions for arbitrary triangulated categories (see also [65] for parallel work by Nicolás, Saorin and Zvonareva on this topic). We also introduce the dual notion of a cosilting object, the 2-term version of which is independently dealt with in [19].

Among silting and cosilting objects, tilting and cotilting objects play a special role: they are the ones providing derived equivalences. Indeed, we show (see Proposition 5.1) that realisation functors associated with silting or cosilting t-structures are fully faithful (and, thus, equivalences with their essential images) if and only if the t-structures are in fact tilting or cotilting. As a consequence of this fact we are then able to establish a derived Morita theory for abelian categories with a projective generator or an injective cogenerator. We refer to Definitions 3.15 and 4.15, as well as to the examples thereafter, for the meaning of restrictable equivalence and bounded (co)tilting (these are conditions that allow restrictions to the setting of bounded derived categories).

Theorem A (5.3) Let $\mathscr{A}$ and $\mathscr{B}$ be abelian categories such that $\mathrm{D}(\mathscr{A})$ is TR5 (respectively, TR $5^{*}$ ) and $\mathscr{B}$ has a projective generator (respectively, an injective cogenerator). Consider the following statements.

(i) There is a restrictable triangle equivalence $\Phi: \mathrm{D}(\mathscr{B}) \longrightarrow \mathrm{D}(\mathscr{A})$.

(ii) There is a bounded tilting (respectively, cotilting) object $M$ in $\mathrm{D}(\mathscr{A})$ such that $\mathcal{H}_{M} \cong \mathscr{B}$.

(iii) There is a triangle equivalence $\phi: \mathrm{D}^{b}(\mathscr{B}) \longrightarrow \mathrm{D}^{b}(\mathscr{A})$.

Then we have (i) $\Longrightarrow$ (ii) $\Longrightarrow$ (iii). Moreover, if $\mathscr{B}$ has a projective generator and $\mathscr{A}=$ Mod- $R$, for a ring $R$, then we also have (iii) $\Longrightarrow$ (ii).

Note that, in particular, Theorem A provides a derived Morita theory for Grothendieck abelian categories, thus covering derived equivalences between not only module categories but also categories of quasicoherent sheaves or certain functor categories, for example. Again, this result stresses that in order to have a derived equivalence arising from a possibly noncompact tilting object, one should look to its heart rather than to its endomorphism ring. In the compact case it so happens that both provide the same information about the derived category but as shown in [12,23], for example, the endomorphism ring of a large tilting module will, in general, provide a recollement rather than a derived equivalence.

It is often easier to know about the existence of a derived equivalence rather than a concrete expression (or shape, for short) for such a functor. Realisation functors satisfy certain naturality properties (see Theorem 3.13, recalling [15, Lemma A7.1]) that contribute to the problem of comparing different equivalence functors and establishing a standard form. Although this problem was solved in algebraic geometry (every equivalence between derived categories of coherent sheaves between two smooth projective varieties is a FourierMukai transform - see [66]), in representation theory it is wide open. For algebras which are projective over a commutative ring, Rickard showed that for every equivalence between derived module categories, there is one of standard type, i.e. one that is the derived tensor product with a complex of bimodules [72]. It remains a question whether every derived equivalence is of standard type, as conjectured by Rickard. There are indications that this should hold. Recently, it was shown in [24] and [25] that derived equivalences between certain finite dimensional algebras (including piecewise hereditary algebras and some Frobenius algebras of radical square zero) are indeed of standard type. In this paper, we prove that all these derived equivalences are, in essence, realisation functors associated to tilting or cotilting objects. Although realisation functors are not unique, we provide new criteria for an 
equivalence to be of standard type and we show that some realisation functors are of standard type.

Theorem $\mathbf{B}$ (5.13) Let $A$ and $B$ be $\mathbb{K}$-algebras which are projective over a commutative ring $\mathbb{K}$. Let $T$ be a compact tilting object in $\mathrm{D}(A)$ such that $\operatorname{End}_{\mathrm{D}(A)}(T) \cong B$. Then the functor real $_{T}$ is an equivalence of standard type. Moreover, a triangle equivalence $\phi: \mathrm{D}^{b}(B) \longrightarrow$ $\mathrm{D}^{b}(A)$ is of standard type if and only if $\phi$ admits an f-lifting $\Phi: \mathrm{DF}^{b}(B) \longrightarrow \mathrm{DF}^{b}(A)$ to the filtered bounded derived categories.

Finally, we discuss recollements and equivalences between them using, once again, realisation functors. Recollements of abelian or triangulated categories are specially well-behaved decompositions (in particular, short exact sequences) of the underlying category. Recollements of abelian categories are well-understood [32], especially if all terms are categories of modules over a ring [70]. The same cannot be said about recollements of derived categories, even in the case where all categories are derived module categories. A natural question (formulated by $\mathrm{Xi}$ for derived module categories) is whether every recollement of derived categories is equivalent to a derived version of a recollement of abelian categories. This is not true in general as shown by a counterexample in [7]. In this paper, we use realisation functors to provide a criterion for such an equivalence of recollements to exist in terms of the glueing of tilting t-structures. A different criterion for recollements of derived categories of rings has been independently obtained in [7]. In the case of a recollement by derived module categories for algebras which are projective over a commutative ring, we prove the following result, which can be thought of as a statement about glueing derived equivalences.

Theorem $\mathbf{C}$ (6.14) Let $A, B$ and $C$ be $\mathbb{K}$-algebras over a commutative ring $\mathbb{K}$ and assume that $A$ is projective as a $\mathbb{K}$-module. Suppose there is a recollement $\mathcal{R}$ of the form

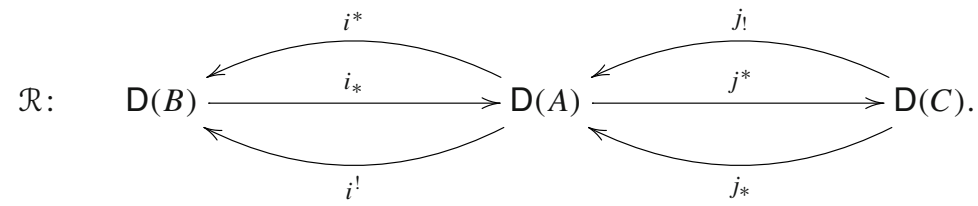

The following statements are equivalent.

(i) There is a $\mathbb{K}$-algebra $S$, projective over $\mathbb{K}$, and an idempotent element e of $S$ such that the canonical ring epimorphism $S \longrightarrow S / S e S$ is homological and the associated recollement of $\mathrm{D}(S)$ by derived module categories is equivalent to $\mathcal{R}$.

(ii) There are compact tilting objects $V$ in $\mathrm{D}(A), U$ in $\mathrm{D}(B)$ and $W$ in $\mathrm{D}(C)$ such that the associated tilting $t$-structures in $\mathrm{D}(B)$ and $\mathrm{D}(C)$ glue along $\mathcal{R}$ to the associated tilting $t$-structure in $\mathrm{D}(A)$ and such that the $\mathbb{K}$-algebra $\operatorname{End}_{\mathrm{D}(A)}(V)$ is projective over $\mathbb{K}$.

\subsection{Structure of the paper}

This paper is organised, roughly, in a sequential way. Sections 3 and 4 are independent of each other, but they are both essential for Sect. 5. Section 6 uses results from all preceding sections. In order to facilitate the understanding of the later sections (where our main results lie) we include in the beginning of each section an informal overview of its results, for the reader that might wish to skip some of the earlier material.

We begin in Sect. 2 with some preliminaries on t-structures, recollements and the relation between the two: glueing. These are the well-known concepts that we will use throughout 
the paper. Section 3 discusses some technical but necessary issues regarding the construction of realisation functors, combining the approach of [16] as well as that presented in [15, Appendix]. We explore at length all the necessary properties for the later sections, including some proofs (or sketches of proof) of older results not available or hard to find in the literature. The results in this section are then used throughout Sects. 5 and 6, where we apply these properties to study equivalences between derived categories of abelian categories or, more generally, between recollements of derived categories. We also show that the realisation functor of the standard t-structure is, as expected, the identity functor. In Sect. 4 we develop our generalised notion of silting and cosilting objects in triangulated categories and we study the properties of the associated hearts. We introduce the notion of bounded silting and bounded cosilting objects, preparing ground for a discussion regarding the relation between derived equivalences at the bounded level and at the unbounded level. This is a recurrent issue throughout the paper, related with the fact that a realisation functor has as domain a bounded derived category. In Sect. 5, we focus on derived equivalences between certain types of abelian categories, both on their existence and on their shape, in the spirit of the above paragraphs. Examples related with the representation theory of infinite quivers and with derived equivalences in algebraic geometry are also discussed. Finally, in Sect. 6 we study recollements of unbounded derived categories: methods to generate them and equivalences between them. We provide criteria in a rather general framework for a recollement of derived categories to be the derived version of a recollement of abelian categories. At the end, as an application, we show that this is always the case for derived categories of hereditary finite dimensional algebras.

This paper also includes an appendix by Ester Cabezuelo Fernández and Olaf M. Schnürer. It discusses a detailed proof for the fact that the realisation functor built as in [15, Appendix] is indeed a triangle functor. For this purpose, however, it seems necessary to consider an extra axiom, as first proposed in [74], for filtered enhancements of triangulated categories. We refer to Remark 3.10 and to Appendix A for a detailed discussion.

\section{Preliminaries: t-structures, recollements and glueing}

\subsection{Conventions and notation}

In this paper we consider only abelian categories with the property that the derived category has Hom-sets. In most contexts, however, the derived categories occurring here come from abelian categories with either enough injectives or enough projectives-and these will have Hom-sets. Given an abelian category $\mathscr{A}$, we denote by $\mathrm{D}(\mathscr{A})$ its derived category. If $A$ is a unitary ring, we denote by Mod- $A$ the category of right $A$-modules and by $\mathrm{D}(A)$ its derived category. Right bounded, left bounded or bounded derived categories are denoted as usual by $\mathrm{D}^{-}, \mathrm{D}^{+}$and $\mathrm{D}^{b}$, respectively. For any triangulated category $\mathcal{T}$, we denote by [1] its suspension functor.

For a category $\mathscr{C}$, we denote by $\mathrm{Ob} \mathscr{C}$ its class of objects. The word subcategory, unless otherwise stated, stands for a full and strict subcategory. For an additive functor $F: \mathscr{A} \longrightarrow \mathscr{B}$ between additive categories the essential image of $F$ is the subcategory of $\mathscr{B}$ given by Im $F=\{B \in \mathscr{B} \mid B \cong F(A)$ for some $A \in \mathscr{A}\}$ and the kernel of $F$ is the subcategory of $\mathscr{A}$ given by $\operatorname{Ker} F=\{A \in \mathscr{A} \mid F(A)=0\}$. If $F$ is a right exact (respectively, left exact) functor between abelian categories, we denote its left derived functor by $\mathbb{L} F$ (respectively, $\mathbb{R} F$ ). If $F$ is exact, its derived functor will often be also denoted by $F$. 


\section{2 t-structures}

We begin with recalling the definition of the key notion of this paper.

Definition 2.1 [16] A t-structure in a triangulated category $\mathcal{T}$ is a pair $\mathbb{T}=\left(\mathbb{T} \leq 0, \mathbb{T}^{\geq 0}\right)$ of full subcategories such that, for $\mathbb{T}^{\leq n}:=\mathbb{T}^{\leq 0}[-n]$ and $\mathbb{T}^{\geq n}:=\mathbb{T}^{\geq 0}[-n](n \in \mathbb{Z})$, we have:

(i) $\operatorname{Hom}_{\mathcal{T}}\left(\mathbb{T}^{\leq 0}, \mathbb{T}^{\geq 1}\right)=0$, i.e. $\operatorname{Hom}_{\mathcal{T}}(X, Y)=0$ for all $X$ in $\mathbb{T}^{\leq 0}$ and $Y$ in $\mathbb{T}^{\geq 1}$;

(ii) $\mathbb{T}^{\leq 0} \subseteq \mathbb{T}^{\leq 1}$ and $\mathbb{T}^{\geq 1} \subseteq \mathbb{T}^{\geq 0}$;

(iii) For every object $X$ in $\mathcal{T}$ there are $Y$ in $\mathbb{T}^{\leq 0}, Z$ in $\mathbb{T}^{\geq 1}$ and a triangle $Y \longrightarrow X \longrightarrow$ $Z \longrightarrow Y[1]$

The subcategories $\mathbb{T}^{\leq 0}, \mathbb{T}^{\geq 0}$ and $\mathcal{H}(\mathbb{T}):=\mathbb{T}^{\leq 0} \cap \mathbb{T}^{\geq 0}$ are called, respectively, the aisle, the coaisle and the heart of $\mathbb{T}$.

It follows from [16] that the heart of a t-structure $\mathbb{T}$ in $\mathcal{T}$ is an abelian category with the exact structure induced by the triangles of $\mathcal{T}$ lying in $\mathcal{H}(\mathbb{T})$. Furthermore, there is a cohomological functor (i.e. a functor sending triangles in $\mathcal{T}$ to long exact sequences in $\mathcal{H}(\mathbb{T})$ ) defined by:

$$
\mathrm{H}_{\mathbb{T}}^{0}: \mathcal{T} \longrightarrow \mathcal{H}(\mathbb{T}), X \mapsto \mathrm{H}_{\mathbb{T}}^{0}(X):=\tau^{\geq 0} \tau^{\leq 0}(X) \cong \tau^{\leq 0} \tau^{\geq 0}(X),
$$

where $\tau^{\leq 0}: \mathcal{T} \longrightarrow \mathbb{T}^{\leq 0}$ and $\tau^{\geq 0}: \mathcal{T} \longrightarrow \mathbb{T}^{\geq 0}$ are the truncation functors (i.e. the right and left adjoins, respectively, of the inclusions of $\mathbb{T}^{\leq 0}$ and $\mathbb{T}^{\geq 0}$ in $\mathcal{T}$ ). Similarly, one can define functors $\tau^{\leq n}, \tau^{\geq n}$ and $H_{\mathbb{T}}^{n}:=\left(\tau^{\leq n} \tau^{\geq n}\right)[n]$, for any integer $n$. The triangle in Definition 2.1(iii) can be expressed functorially as

$$
\tau^{\leq 0} X \stackrel{f}{\longrightarrow} X \stackrel{g}{\longrightarrow} \tau^{\geq 1} X \longrightarrow\left(\tau^{\leq 0} X\right)[1]
$$

where the maps $f$ and $g$ come, respectively, from the counit and unit of the relevant adjunctions. In particular, it follows that if $f=0$ (respectively, $g=0$ ), then $\tau^{\leq 0} X=0$ (respectively, $\tau^{\geq 1} X=0$ ). Note also that the aisle $\mathbb{T}^{\leq 0}$ determines the t-structure $\left(\mathbb{T}^{\geq 1}=\operatorname{KerHom}_{\mathcal{T}}\left(\mathbb{T}^{\leq 0},-\right)\right.$, see also [50]).

Example 2.2 For an abelian category $\mathscr{A}$, there is a standard t-structure $\mathbb{D}_{\mathscr{A}}:=\left(\mathbb{D}_{\mathscr{A}}^{\leq 0}, \mathbb{D}_{\mathscr{A}}^{\leq 0}\right)$ in its derived category $\mathrm{D}(\mathscr{A})$ defined by the complex cohomology functors $\left(\mathrm{H}_{0}^{i}\right.$, for all $i$ in $\mathbb{Z})$ as follows:

$\mathbb{D}_{\mathscr{A}}^{\leq 0}:=\left\{X \in \mathrm{D}(\mathscr{A}) \mid \mathrm{H}_{0}^{i}(X)=0, \forall i>0\right\}, \quad \mathbb{D}_{\mathscr{A}}^{\geq 0}:=\left\{X \in \mathrm{D}(\mathscr{A}) \mid \mathrm{H}_{0}^{i}(X)=0, \forall i<0\right\}$.

The heart of this t-structure consists of the complexes with cohomologies concentrated in degree zero and, thus, it is equivalent to $\mathscr{A}$. Moreover, the associated cohomology functors coincides with the complex cohomologies. Note also that the standard t-structure restricts to the (right, left) bounded derived category $\mathrm{D}^{b}(\mathscr{A})$. Throughout we fix the notation in this example for the standard t-structure, although the subscript $\mathscr{A}$ will be omitted whenever $\mathscr{A}$ is fixed.

A t-structure $\mathbb{T}=\left(\mathbb{T}^{\leq 0}, \mathbb{T}^{\geq 0}\right)$ in $\mathcal{T}$ is said to be nondegenerate if $\cap_{n \in \mathbb{Z}} \mathrm{Ob} \mathbb{T}^{\leq n}=0=$ $\cap_{n \in \mathbb{Z}} \mathrm{Ob} \mathbb{T}^{\geq n}$ and bounded if $\cup_{n \in \mathbb{Z}} \mathrm{Ob} \mathbb{T}^{\leq n}=\mathrm{Ob} \mathcal{T}=\cup_{n \in \mathbb{Z}} \mathrm{Ob} \mathbb{T}^{\geq n}$. Given an abelian category $\mathscr{A}$, it is easy to see that the standard t-structure is nondegenerate in both $\mathrm{D}^{b}(\mathscr{A})$ and $\mathrm{D}(\mathscr{A})$, but bounded only in $\mathrm{D}^{b}(\mathscr{A})$.

For functors between triangulated categories endowed with t-structures there is a natural notion of (left, right) exactness. Given two triangulated categories $\mathcal{T}$ and $\mathcal{V}$ endowed with t-structures $\mathbb{T}=\left(\mathbb{T}^{\leq 0}, \mathbb{T}^{\geq 0}\right)$ and $\mathbb{V}=(\mathbb{V} \leq 0, \mathbb{V} \geq 0)$ respectively, a functor $\alpha: \mathcal{T} \longrightarrow \mathcal{V}$ is 
said to be left $\mathbf{t}$-exact if $\alpha\left(\mathbb{T}^{\geq 0}\right) \subseteq \mathbb{V} \geq 0$, right $\mathbf{t}$-exact if $\alpha\left(\mathbb{T}^{\leq 0}\right) \subseteq \mathbb{V} \leq 0$ and t-exact if it is both left and right t-exact. As an example, consider two abelian categories $\mathscr{A}$ and $\mathscr{B}$ and a functor $F: \mathscr{A} \longrightarrow \mathscr{B}$. If $F$ is exact, then its derived functor is t-exact with respect to the standard t-structures in $\mathrm{D}(\mathscr{A})$ and $\mathrm{D}(\mathscr{B})$. If $\mathscr{A}$ has enough projectives (respectively, injectives) and $F$ is right (respectively, left) exact, then the left derived functor $\mathbb{L} F$ is right t-exact (respectively, the right derived functor $\mathbb{R} F$ is left t-exact).

\subsection{Recollements}

Recall first that a diagram of the form

$$
0 \longrightarrow \mathcal{U} \stackrel{i}{\longrightarrow} \mathcal{T} \stackrel{q}{\longrightarrow} \mathcal{V} \longrightarrow 0
$$

is said to be a short exact sequence of triangulated (respectively, abelian) categories if the functor $i$ is fully faithful, $i(\mathcal{U})$ is a thick subcategory of $\mathcal{T}$, i.e. a triangulated subcategory closed under summands (respectively, a Serre subcategory in the abelian case, i.e. a subcategory closed under extensions, subobjects and quotients) and if $q$ induces an equivalence $\mathcal{T} / i(\mathcal{U}) \cong \mathcal{V}$. Note that, in this case, $\operatorname{Im} i=\operatorname{Ker} q$ and $q$ is essentially surjective.

Recollements are particularly well-behaved short exact sequences. Recollements of both abelian and triangulated categories appeared in [16], but the properties of recollements of abelian categories were only explored later, for example in [32].

Definition 2.3 Let $\mathcal{U}, \mathcal{T}$ and $\mathcal{V}$ be triangulated (respectively, abelian) categories. A recollement of $\mathcal{T}$ by $\mathcal{U}$ and $\mathcal{V}$ is a diagram of triangle (respectively, additive) functors

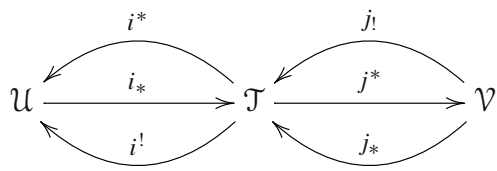

satisfying the following conditions:

(i) $\left(i^{*}, i_{*}, i^{!}\right)$and $\left(j !, j^{*}, j_{*}\right)$ are adjoint triples;

(ii) The functors $i_{*}, j_{!}$, and $j_{*}$ are fully faithful;

(iii) $\operatorname{Im} i_{*}=\operatorname{Ker} j^{*}$.

Generally, we will use the symbols $\mathcal{U}, \mathcal{T}$ and $\mathcal{V}$ to denote triangulated categories (and we write $\mathrm{R}_{\mathrm{tr}}(\mathcal{U}, \mathcal{T}, \mathcal{V})$ for the recollement in Definition 2.3) and the symbols $\mathscr{B}, \mathscr{A}$ and $\mathscr{C}$ to denote abelian categories (and we write $\mathrm{R}_{\mathrm{ab}}(\mathscr{B}, \mathscr{A}, \mathscr{C}$ ) for the analogous recollement to the one in Definition 2.3). Observe that it follows easily from the definition of recollement that the compositions $i^{*} j_{\text {! }}$ and $i^{!} j_{*}$ are identically zero and that the units Id $\longrightarrow i^{!} i_{*}$ and Id $\longrightarrow j^{*} j_{\text {! }}$ and the counits $i^{*} i_{*} \longrightarrow$ Id and $j^{*} j_{*} \longrightarrow$ Id of the adjunctions are natural isomorphisms (where the identity functors are defined in the obvious categories). Moreover, it can also be shown (see, for example, $[16,69]$ ) that for a recollement of triangulated categories $\mathrm{R}_{\operatorname{tr}}(\mathcal{U}, \mathcal{T}, \mathcal{V})$, for every object $X$ in $\mathcal{T}$, the remaining units and the counits of the adjunctions induce triangles

$$
j ! j^{*} X \longrightarrow X \longrightarrow i_{*} i^{*} X \longrightarrow j ! j^{*} X[1] \text { and } i_{*} i^{!} X \longrightarrow X \longrightarrow j_{*} j^{*} X \longrightarrow i_{*} i^{!} X[1] .
$$

Similarly, for a recollement of abelian categories $\mathrm{R}_{\mathrm{ab}}(\mathscr{B}, \mathscr{A}, \mathscr{C})$, for every object $X$ in $\mathscr{A}$, the remaining units and counits of the adjunctions induce exact sequences:

$$
j ! j^{*} X \stackrel{f}{\longrightarrow} X \longrightarrow i_{*} i^{*} X \longrightarrow 0 \text { and } 0 \longrightarrow i_{*} i^{!} X \longrightarrow X \stackrel{g}{\longrightarrow} j_{*} j^{*} X
$$


with Ker $f$ and Coker $g$ lying in $i_{*} \mathscr{B}$ (see, for example, [32] and [69] for details).

A useful tool to produce recollements of module categories or of derived module categories is the concept of ring epimorphsim, i.e. an epimorphism in the category of (unital) rings. It is well-known (see [34,35]) that ring epimorphisms with domain $R$ (up to the natural notion of equivalence) are in bijection with bireflective subcategories of Mod- $R$, i.e. full subcategories of Mod- $R$ whose inclusion functor admits both left and right adjoints. In order for the (exact) restriction of scalars functor to induce a fully faithful functor on the derived level, however, one needs to require more from a ring epimorphism.

Theorem 2.4 [35, Theorem 4.4][64, Section 4] Let $A$ be a ring and $f: A \longrightarrow B$ a ring homomorphism. The following are equivalent.

(i) The derived functor $f_{*}: \mathrm{D}(B) \longrightarrow \mathrm{D}(A)$ is fully faithful.

(ii) The map $f$ is a ring epimorphism and, for any $i>0$, we have $\operatorname{Tor}_{i}^{A}(B, B)=0$.

(iii) For any $i \geq 0$, and any $B$-modules $M$ and $N$, we have $\operatorname{Ext}_{B}^{i}(M, N)=\operatorname{Ext}_{A}^{i}(M, N)$.

We say that a ring epimorphism is homological if the above equivalent conditions are satisfied. Note that while ring epimorphisms do not always give rise to recollements of module categories (see [70]), a homological ring epimorphism always gives rise to a recollement of triangulated categories (see [64]).

Example 2.5 Let $A$ be a ring, $e$ an idempotent element of $A$ and $f: A \longrightarrow A / A e A$ the associated ring epimorphism. There is a recollement of Mod- $A$, as in the diagram below, which is said to be induced by the idempotent element $e$.

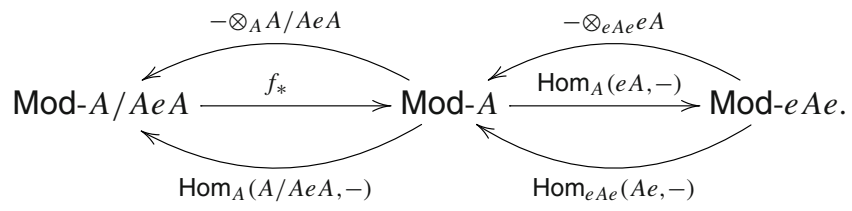

Moreover, if $\operatorname{Tor}_{i}^{A}(A / A e A, A / A e A)=0$ for all $i>0$ (i.e. $f$ is a homological ring epimorphism), it follows from [27] that there is a recollement of triangulated categories:

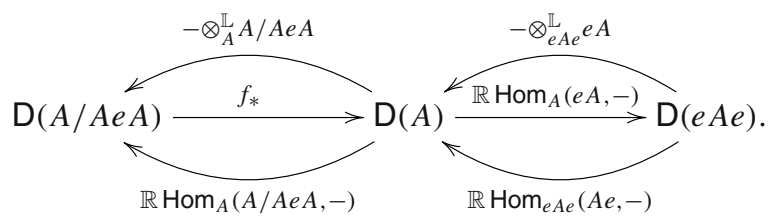

Definition 2.6 We say that a recollement of a derived module category by derived module categories is stratifying if it is of the form (2.1).

We study recollements up to the following notion of equivalence (see also [70]). Throughout the paper, a diagram of functors is said to be commutative if it commutes up to natural equivalence of functors.

Definition 2.7 Two recollements of triangulated categories $R_{\operatorname{tr}}(\mathcal{U}, \mathcal{T}, \mathcal{V})$ and $R_{\operatorname{tr}}\left(\mathcal{U}^{\prime}, \mathcal{T}^{\prime}, \mathcal{V}^{\prime}\right)$ are equivalent, if there are triangle equivalence functors $F: \mathcal{T} \longrightarrow \mathcal{T}^{\prime}$ and $G: \mathcal{V} \longrightarrow \mathcal{V}^{\prime}$ such 
that the diagram below commutes, i.e. there is a natural equivalence of functors $G j^{*} \cong j^{* \prime} F$.

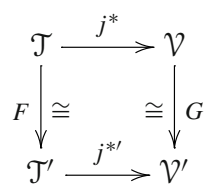

An equivalence of recollements of abelian categories is defined analogously.

Note that the commutativity (up to natural equivalences) of the above diagram is equivalent to the commutativity of the six diagrams associated to the six functors of the recollements (see [70, Lemma 4.2] for more details on the abelian case; the triangulated case is analogous, see [68]).

\subsection{Glueing}

Example 2.5 shows how sometimes it is possible to build a recollement of triangulated categories from a recollement of abelian categories. In this subsection we recall from [16] a procedure in the opposite direction, using t-structures.

Theorem 2.8 [16] Let $R_{\mathrm{tr}}(\mathcal{U}, \mathcal{T}, \mathcal{V})$ be a recollement of triangulated categories of the form (2.3). Suppose that $\mathbb{U}=\left(\mathbb{U} \leq 0, \mathbb{U}^{\geq 0}\right)$ and $\mathbb{V}=(\mathbb{V} \leq 0, \mathbb{V} \geq 0)$ are $t$-structures in $\mathcal{U}$ and $\mathcal{V}$, respectively. Then there is a $t$-structure $\mathbb{T}=\left(\mathbb{T}^{\leq 0}, \mathbb{T}^{\geq 0}\right)$ in $\mathcal{T}$ defined by

$$
\begin{aligned}
& \mathbb{T}^{\leq 0}=\left\{X \in \mathcal{T} \mid j^{*}(X) \in \mathbb{V} \leq 0 \text { and } i^{*}(X) \in \mathbb{U}^{\leq 0}\right\}, \\
& \mathbb{T}^{\geq 0}=\left\{X \in \mathcal{T} \mid j^{*}(X) \in \mathbb{V} \geq 0 \text { and } i^{!}(X) \in \mathbb{U}^{\geq 0}\right\} .
\end{aligned}
$$

Convesely, given a t-structure $\mathbb{T}=\left(\mathbb{T}^{\leq 0}, \mathbb{T}^{\geq 0}\right)$ in $\mathcal{T}, \mathbb{T}$ is obtained as above from $t$-structures $\mathbb{U}$ and $\mathbb{V}$ in $\mathcal{U}$ and $\mathcal{V}$, respectively, if and only if $j_{!} j^{*} \mathbb{T} \leq 0 \subseteq \mathbb{T} \leq 0$. In that case, $\mathbb{U}$ and $\mathbb{V}$ are uniquely determined by $\mathbb{U}=\left(i^{*} \mathbb{T} \leq 0, i ! \mathbb{T}^{\geq 0}\right)$ and $\mathbb{V}=\left(j^{*} \mathbb{T} \leq 0, j^{*} \mathbb{T}^{\geq 0}\right)$, the functors $i_{*}$ and $j^{*}$ are t-exact and their left (respectively, right) adjoints are right (respectively, left) t-exact. Moreover, the recollement of triangulated categories $R_{\mathrm{tr}}(\mathcal{U}, \mathcal{T}, \mathcal{V})$ induces a recollement of abelian categories of the corresponding hearts $R_{\mathrm{ab}}(\mathcal{H}(\mathbb{U}), \mathcal{H}(\mathbb{T}), \mathcal{H}(\mathbb{V}))$.

We explain how to build the recollement $R_{a b}(\mathcal{H}(\mathbb{U}), \mathcal{H}(\mathbb{T}), \mathcal{H}(\mathbb{V}))$ from $R_{\mathrm{tr}}(\mathcal{U}, \mathcal{T}, \mathcal{V})$, as stated in the theorem. Consider the cohomological functors $\mathrm{H}_{\mathbb{U}}^{0}: \mathcal{U} \longrightarrow \mathcal{H}(\mathbb{U}), \mathrm{H}_{\mathbb{T}}^{0}: \mathcal{T} \longrightarrow$ $\mathcal{H}(\mathbb{T}), \mathrm{H}_{\mathbb{V}}^{0}: \mathcal{V} \longrightarrow \mathcal{H}(\mathbb{V})$, and the full embeddings $\varepsilon_{\mathbb{U}}: \mathcal{H}(\mathbb{U}) \longrightarrow \mathcal{U}, \varepsilon_{\mathbb{T}}: \mathcal{H}(\mathbb{T}) \longrightarrow$ $\mathcal{T}, \varepsilon_{\mathbb{V}}: \mathcal{H}(\mathbb{V}) \longrightarrow \mathcal{V}$ associated with the t-structures $\mathbb{U}, \mathbb{T}$ and $\mathbb{V}$, respectively. Then the recollement $\mathrm{R}_{\mathrm{ab}}(\mathcal{H}(\mathbb{U}), \mathcal{H}(\mathbb{T}), \mathcal{H}(\mathbb{V}))$ is given by

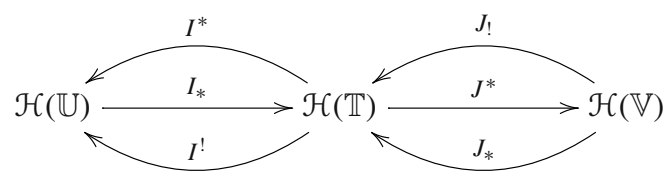

where the functors are defined as follows:

$$
\begin{array}{rlrl}
I^{*} & =\mathrm{H}_{\mathbb{U}}^{0} \circ i^{*} \circ \epsilon_{\mathbb{T}} & J_{*}=\mathrm{H}_{\mathbb{T}}^{0} \circ j ! \circ \epsilon_{\mathbb{V}} \\
I_{*}=\mathrm{H}_{\mathbb{T}}^{0} \circ i_{*} \circ \epsilon_{\mathbb{U}} & J^{*}=\mathrm{H}_{\mathbb{V}}^{0} \circ j^{*} \circ \epsilon_{\mathbb{T}} \\
I^{!}=\mathrm{H}_{\mathbb{U}}^{0} \circ i^{!} \circ \epsilon_{\mathbb{T}} & J_{*}=\mathrm{H}_{\mathbb{T}}^{0} \circ j_{*} \circ \epsilon_{\mathbb{V} .}
\end{array}
$$


In other words, we have the following diagram:
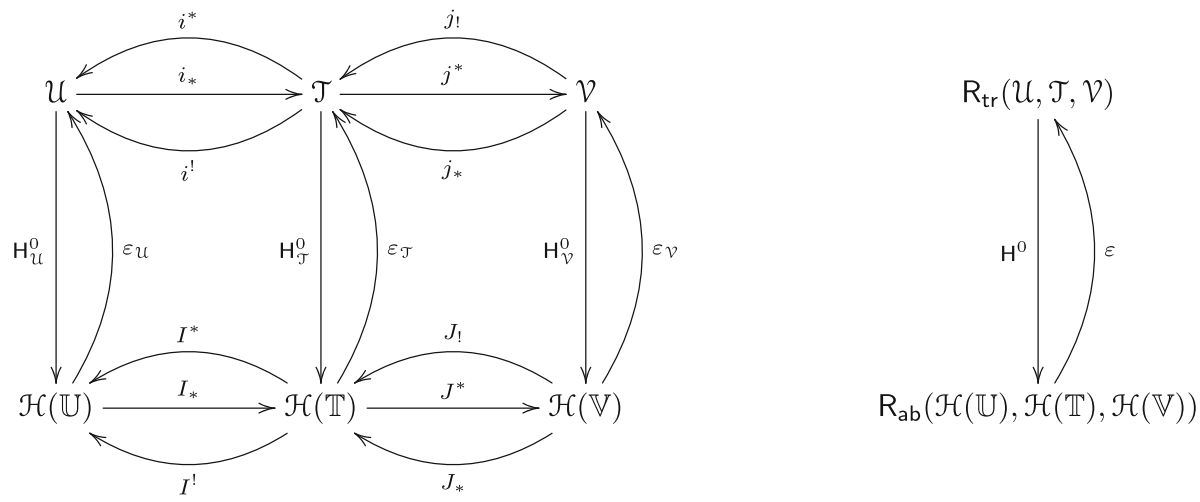

where the functors in the lower recollement are defined using the vertical functors as described above. A recollement obtained in this way will be called a recollement of hearts.

\section{Realisation functors}

Given a t-structure in a triangulated category $\mathcal{T}$ with heart $\mathcal{H}$, it is natural to ask how does the (bounded) derived category of $\mathcal{H}$ compare with $\mathcal{T}$. In [16], a functor between these two categories is built under some assumptions on the category $\mathcal{T}$ and the t-structure on it: the realisation functor. We consider the more general approach from [15] that allows the construction of the realisation functor for t-structures in any triangulated category that admits an f-category over it. We survey this construction and the relevant associated notions in some detail, as we will need a deeper understanding of this functor later in this text. We refer to [49] for a different approach to the realisation functor. Here is an informal overview of this section.

\section{Subsection 3.1: f-Categories}

- We review the definition of a filtered enhancement of a triangulated category, motivated by the example of filtered derived categories (Example 3.2).

- We recall how to lift a t-structure from a triangulated category to a filtered enhancement (Proposition 3.3). This is an important step towards the construction of realisation functors.

- We show that given a triangulated category with a filtered enhancement, there are compatible filtered enhancements on any thick subcategory and on any Verdier quotient (Proposition 3.8). This results is useful for the use of realisation functors in the context of recollements (Sect. 6).

\section{Subsection 3.2: Realisation functors and their properties}

- We recall with some detail the construction and basic properties of realisation functors (Theorem 3.11) which will be used throughout the paper.

- We discuss a result of Beilinson concerning commutative diagrams of functors involving realisation functors (Theorem 3.13). This is particularly relevant for the study of the 
shape of derived equivalences in Sect. 5 and for the construction of equivalences of recollements in Sect. 6.

\section{Subsection 3.3: Examples of realisation functors}

- We show that the realisation functor of the standard t-structure in a derived category (built with respect to the filtered derived category) is essentially an identity functor (Proposition 3.14).

- Given a triangle equivalence $\phi$ between the bounded derived categories of two abelian categories, we show that $\phi$ is naturally equivalent to a realisation functor composed with the derived functor of an exact equivalence of abelian categories (Proposition 3.17).

\section{1 f-Categories}

The key idea for constructing a realisation functor in [16] was that of using the so-called filtered derived category. However, as it was observed in [15], we only need the abstract properties of such categories for this construction-giving rise to the notion of an f-category. For a detailed survey on f-categories, we refer to [74].

Definition 3.1 An f-category is a triangulated category $X$ endowed with an autoequivalence $s: X \longrightarrow X$ (called $f$-shift), a natural transformation $\alpha: \operatorname{Id} X \longrightarrow s$ and two full triangulated subcategories $X(\geq 0)$ and $X(\leq 0)$ such that, for $X(\geq n):=s^{n} X(\geq 0)$ and $X(\leq n):=s^{n} X(\leq 0)$, we have

(i) $\operatorname{Hom}_{x}(X(\geq 1), X(\leq 0))=0$;

(ii) For every object $X$ in $X$, there are $Y$ in $X(\geq 1), Z$ in $X(\leq 0)$ and a triangle in $X$ :

$$
Y \longrightarrow X \longrightarrow Z \longrightarrow Y[1]
$$

(iii) $\mathrm{Ob} X=\cup_{n \in \mathbb{Z}} \mathrm{Ob} X(\geq n)=\cup_{n \in \mathbb{Z}} \mathrm{Ob} X(\leq n)$;

(iv) $x(\geq 1) \subseteq x_{(\geq 0)}$ and $x_{(\leq-1)} \subseteq x_{(\leq 0)}$;

(v) $\alpha_{X}=s\left(\alpha_{s^{-1} X}\right)$ for all $X$ in $X$;

(vi) For any $X \in X(\geq 1)$ and $Y \in X(\leq 0), \operatorname{Hom}_{X}\left(\alpha_{Y}, X\right)$ and $\operatorname{Hom}_{X}\left(Y, \alpha_{X}\right)$ are isomorphisms.

Given a triangulated category $\mathcal{T}$, an f-category over $\mathcal{T}$ (or an f-enhancement of $\mathcal{T}$ ) is a pair $(X, \theta)$ where $X$ is an f-category and $\theta: \mathcal{T} \longrightarrow X(\geq 0) \cap X(\leq 0)$ is an equivalence of triangulated categories.

For an f-category $X$, we write the whole data as $(X, X(\geq 0), X(\leq 0), s: X \stackrel{\cong}{\longrightarrow}$ $X, \alpha: \operatorname{Id} X \longrightarrow s)$, although we write just $X$ when the remaining data is fixed. Let $(X, \theta)$ denote an f-category over $\mathcal{T}$. Note that for any $n$ in $\mathbb{Z}$, the pair $(\mathcal{X}(\geq n+1), \mathcal{X}(\leq n))$ is a stable t-structure, i.e. a t-structure whose aisle is a triangulated subcategory. In particular, there are truncation functors $\sigma_{\geq n}: X \longrightarrow X(\geq n)$ and $\sigma_{\leq n}: X \longrightarrow X(\leq n)$, which are triangle functors. We define the following further triangle functors

$$
\operatorname{gr}_{X}^{n}:=\theta^{-1} s^{-n} \sigma_{\leq n} \sigma_{\geq n}: X \longrightarrow \mathcal{T}
$$

There are standard f-categories over a large class of triangulated categories: (bounded) derived categories of abelian categories. These are the so-called filtered derived categories. In the following example we build the filtered derived category of the unbounded derived category of an abelian category. The bounded setting is entirely analogous. 
Example 3.2 [42] Given an abelian category $\mathscr{A}$, consider the (additive) category $\mathrm{CF}(\mathscr{A})$ of complexes of objects in $\mathscr{A}$ endowed with a finite decreasing filtration. The objects in $\operatorname{CF}(\mathscr{A})$ are, thus, pairs $(X, F)$, where $X$ lies in the category of complexes $\mathrm{C}(\mathscr{A})$ and $F$ is a filtration of $X$ as follows:

$$
X=F_{a} X \supseteq F_{a+1} X \supseteq F_{a+2} X \supseteq \cdots \supseteq F_{b-1} X \supseteq F_{b} X=0,
$$

with $a \leq b$ integers. The morphisms in $\operatorname{CF}(\mathscr{A})$ are morphisms of complexes respecting the filtration, i.e. given two filtered complexes $(X, F)$ and $(Y, G)$ a morphism $f:(X, F) \longrightarrow$ $(Y, G)$ in $\operatorname{CF}(\mathscr{A})$ is a sequence of chain maps $\left(\ldots, F_{a} f, F_{a+1} f, \ldots, F_{b} f, \ldots\right)$, with $F_{i} f: F_{i} X \longrightarrow G_{i} Y$ compatible with the inclusion maps of the filtrations $F$ and $G$. There are natural functors $\mathrm{gr}^{i}: \mathrm{CF}(\mathscr{A}) \longrightarrow \mathrm{C}(\mathscr{A})$ associating to a filtered complex $(X, F)$ its $i$-th graded component $\operatorname{gr}_{F}^{i}(X):=F_{i} X / F_{i+1} X$. A morphism $\phi$ in $\operatorname{CF}(\mathscr{A})$ is said to be a filtered quasi-isomorphism if $F_{i} f$ is a quasi-isomorphism, for all $i$ in $\mathbb{Z}$ (see [42] for further equivalent definitions of filtered quasi-isomorphisms). The filtered derived category $\operatorname{DF}(\mathscr{A})$ of an abelian category $\mathscr{A}$ is the localisation of $\mathrm{CF}(\mathscr{A})$ on filtered quasi-isomorphisms. Moreover, one can also define the filtered homotopy category $\operatorname{KF}(\mathscr{A})$ of $\mathscr{A}$, where the objects are the same as in $\operatorname{CF}(\mathscr{A})$ but the morphisms are equivalences classes of morphisms in $\operatorname{CF}(\mathscr{A})$ modulo filtered homotopy (two morphisms $f, f^{\prime}:(X, F) \longrightarrow(Y, G)$ in $\mathrm{CF}(\mathscr{A})$ are homotopic, if there is a homotopy from $f$ to $f^{\prime}$ compatible with the filtrations). The filtered derived category can also obtained as the localisation of $\operatorname{KF}(\mathscr{A})$ on filtered quasi-isomorphisms.

Note that there is a natural fully faithful functor $\xi: \mathrm{D}(\mathscr{A}) \longrightarrow \mathrm{DF}(\mathscr{A})$ sending an object $X$ in $\mathrm{D}(\mathscr{A})$ to the pair $(X, 0)$, where 0 indicates the trivial filtration $X=F_{0}(X) \supseteq F_{1} X=0$. The filtered derived category comes naturally equipped with an autoequivalence $s: \operatorname{DF}(\mathscr{A}) \longrightarrow$ $\operatorname{DF}(\mathscr{A})$ corresponding to the shift on filtration, i.e. $s(X, F)=(X, G)$, where $G_{i} X=F_{i-1} X$. Also, there is a natural transformation $\alpha: \operatorname{Id}_{\mathrm{DF}(\mathscr{A})} \longrightarrow s$ such that for any object $X, \alpha_{X}$ is induced by the inclusion maps of $F_{i+1} X$ into $F_{i} X$, for all $i$ in $\mathbb{Z}$. Finally, consider $\operatorname{DF}(\mathscr{A})(\geq 0)$ (respectively, $\operatorname{DF}(\mathscr{A})(\leq 0)$ ) to be the full subcategory of $\operatorname{DF}(\mathscr{A})$ spanned by filtered complexes whose non-trivial graded components are in non-negative (respectively, non-positive) degrees. It follows that $\operatorname{DF}(\mathscr{A})$ is an f-category over $\mathrm{D}(\mathscr{A})$. There is also a natural functor $\omega: \operatorname{DF}(\mathscr{A}) \longrightarrow \mathrm{D}(\mathscr{A})$, the forgetful functor. Note also that the functors $\mathrm{gr}^{i}$ defined at the level of complexes induce triangle functors from $\mathrm{DF}(\mathscr{A}) \longrightarrow \mathrm{D}(\mathscr{A})$ and, as the notation suggests, these are the analogues in this setting of the gr-functors defined in the general context of f-categories.

We summarise some useful facts about f-categories over a triangulated category.

Proposition $3.3[15,74]$ If $(X, \theta)$ is an $f$-category over a triangulated category $\mathcal{T}$, then:

(i) there is an exact functor $\omega: X \longrightarrow \mathcal{T}$, unique up to natural equivalence, such that:

- its restriction to $X(\geq 0)$ is right adjoint to the functor $\mathcal{T} \longrightarrow X(\geq 0)$ induced by $\theta$;

- its restriction to $X(\leq 0)$ is left adjoint to the functor $\mathcal{T} \longrightarrow X(\leq 0)$ induced by $\theta$;

- for any $X$ in $X$, the map $\omega\left(\alpha_{X}\right): \omega X \longrightarrow \omega s X$ is an isomorphism;

- for any $X$ in $X(\leq 0)$ and $Y$ in $X(\geq 0)$, $\omega$ induces an isomorphism between $\operatorname{Hom}_{X}(X, Y)$ and $\operatorname{Hom}_{\mathcal{T}}(\omega X, \omega Y)$.

(ii) given a t-structure $\mathbb{T}=\left(\mathbb{T}^{\leq 0}, \mathbb{T}^{\geq 0}\right)$ in $\mathcal{T}$, there is a unique -structure $\mathbb{X}=\left(\mathbb{X} \leq 0, \mathbb{X}^{\geq 0}\right)$ in $\mathcal{X}$ such that $\theta$ is a $t$-exact functor and $s \mathbb{X} \leq 0 \subseteq \mathbb{X} \leq-1$. Moreover, the $t$-structure $\mathbb{X}$ can be described by

$$
\mathbb{X}^{\leq 0}=\left\{X \in X \mid g r_{X}^{n}(X) \in \mathbb{T}^{\leq n}\right\} \text { and } \mathbb{X}^{\geq 0}=\left\{X \in X \mid g r_{X}^{n}(X) \in \mathbb{T}^{\geq n}\right\}
$$


and the heart $\mathcal{H}(\mathbb{X})$ is equivalent to the category $\mathscr{C}^{b}(\mathcal{H}(\mathbb{T}))$ of chain complexes over $\mathcal{H}(\mathbb{T})$.

Remark 3.4 We point out how to build the functor yielding an equivalence between $\mathcal{H}(\mathbb{X})$ and $C^{b}(\mathcal{H}(\mathbb{T}))$ (see [15]). There is a cohomological functor which we will, abusively, denote by $\mathrm{H}_{\mathbb{X}}^{0}: X \longrightarrow \mathrm{C}^{b}(\mathcal{H}(\mathbb{T}))$. Given an object $X$ in $X$, define $\mathrm{H}_{\mathbb{X}}^{0}(X)$ to be a complex whose $i$-th component is $\mathrm{H}_{\mathbb{T}}^{i}\left(\operatorname{gr}_{X}^{i} X\right)$. In order to define the differential $d^{i}: \mathrm{H}_{\mathbb{T}}^{i}\left(\operatorname{gr}_{X}^{i} X\right) \longrightarrow$ $\mathrm{H}_{\mathbb{T}}^{i+1}\left(\operatorname{gr}_{X}^{i+1} X\right)$ consider the triangle in $\mathcal{T}$ given by

$$
\omega \sigma_{\leq i+1} \sigma_{\geq i+1} X \longrightarrow \omega \sigma_{\leq i+1} \sigma_{\geq i} X \longrightarrow \omega \sigma_{\leq i} \sigma_{\geq i} X \stackrel{\bar{d}^{i}}{\longrightarrow}\left(\omega \sigma_{\leq i+1} \sigma_{\geq i+1} X\right)[1] .
$$

Now, the properties of $\omega$ listed above insure that, for any $n$ in $\mathbb{Z}$, we have $\omega \sigma_{\leq n} \sigma_{\geq n} X \cong \operatorname{gr}_{X}^{n} X$. It then follows that we can define $d^{i}$ as $\mathrm{H}_{\mathbb{T}}^{i}\left(\bar{d}^{i}\right)$, for any $i$ in $\mathbb{Z}$. This defines the functor $\mathrm{H}_{\mathbb{X}}^{0}$ and it can be seen that this functor yields an exact equivalence between $\mathcal{H}(\mathbb{X})$ and $C^{b}(\mathcal{H}(\mathbb{T})$ ) as wanted (see [16] for a proof in the case of filtered derived categories; the statement for f-categories is available without proof in [15] since the arguments are analogous). The abuse of notation here is justified by the fact that indeed $\mathrm{H}_{\mathbb{X}}^{0}$ can be regarded as a cohomological functor associated with the t-structure $\mathbb{X}$ in $X$ (see [15]).

The functor $\omega$ in the proposition will be called the f-forgetful functor, as motivated by the actual forgetful functor in the case of filtered derived categories. Note that the existence of f-enhancements of triangulated categories is not a priori guaranteed-although conjectured (see [21]).

We turn now to functors between f-categories.

Definition 3.5 Let $(X, X(\geq 0), X(\leq 0), s, \alpha)$ and $(y, y(\geq 0), y(\leq 0), t, \beta)$ be two $f$-categories. An f-functor between the f-categories $X$ and $y$ is a triangle functor $F: X \longrightarrow Y$ such that:

(i) $F(X(\geq 0)) \subseteq y(\geq 0)$ and $F(X(\leq 0)) \subseteq y(\leq 0)$;

(ii) $F s \cong t F$ and $F\left(\alpha_{X}\right)=\beta_{F(X)}$, for all $X$ in $X$.

The f-categories $X$ and $y$ are equivalent, if there is an f-functor $F: X \longrightarrow Z$ which is a triangle equivalence. If $(\mathcal{X}, \theta)$ and $(\mathcal{Y}, \eta)$ are $\mathrm{f}$-categories over triangulated categories $\mathcal{T}$ and $\mathcal{U}$, respectively and $\phi: \mathcal{T} \longrightarrow \mathcal{U}$ is a triangle functor, we say that $\phi$ lifts to the f-categories $(\mathcal{X}, \theta)$ and $(y, \eta)$ if there is an f-functor $\Phi: X \longrightarrow y$ such that $\Phi \theta \cong \eta \phi$. When the f-categories are fixed, we will just say that $\phi$ admits an f-lifting.

Example 3.6 Given triangulated categories $\mathcal{T}$ and $\mathcal{U}$ and a triangle equivalence $\phi: \mathcal{T} \longrightarrow \mathcal{U}$, if $(\mathcal{X}, \theta)$ is an f-category over $\mathcal{T}$, then $\left(\mathcal{X}, \theta \phi^{-1}\right)$ is an f-category over $\mathcal{U}$ and, hence, $\operatorname{Id} \mathcal{X}$ is an f-lifting of $\phi$.

We will show that given an exact sequence of triangulated categories

$$
0 \longrightarrow \mathcal{U} \longrightarrow \mathcal{T} \longrightarrow \mathcal{T} / \mathcal{U} \longrightarrow 0
$$

and an $f$-category over $\mathcal{T}$, there are induced $\mathrm{f}$-categories over the thick subcategory $\mathcal{U}$ and over the Verdier quotient $\mathcal{T} / \mathcal{U}$, improving on [79, Proposition 2.7]. We say that a thick subcategory $y$ of an f-category $X$ is an f-subcategory if $y$ is an f-category with the induced f-structure, i.e. $y(\leq 0)=x(\leq 0) \cap y, y(\geq 0)=x(\geq 0) \cap y$ and both $s: y \longrightarrow y$ and $\alpha$ : Id $y \rightarrow s$ are the restrictions of the corresponding functor/natural transformation in $X$. 
Lemma 3.7 Let $y$ be a thick $f$-subcategory of an $f$-category $X$. Then the Verdier quotient $X / y$ has a natural $f$-category structure induced by the one in $X$.

Proof Since $y$ is a thick subcategory, there is a short exact sequence of triangulated categories

$$
0 \longrightarrow y \stackrel{j}{\longrightarrow} x \stackrel{p}{\longrightarrow} x / y \longrightarrow 0 \text {. }
$$

Set $Z=X / Y$ and consider the full triangulated subcategories $Z(\geq 0)=p(X(\geq 0))$ and $z(\leq 0)=p(X(\leq 0))$. From the sequence (3.1), one can easily observe that the functor $s: X \longrightarrow X$ induces a functor $s_{Z}: \mathcal{Z} \longrightarrow \mathcal{Z}$. It is obvious that $s_{Z}$ is essentially surjective. It is also fully faithful (and hence an autoequivalence of $Z$ ) since its action on morphisms can be described by applying $s$ to a roof in $z$. Non-trivial roofs will remain non-trivial due to the fact that $s$ restricts as an autoequivalence to $y$ (since $y$ is an f-subcategory). We also obtain an induced natural transformation $\gamma: \operatorname{Id}_{z} \longrightarrow s_{z}$ of triangulated functors. Indeed, using the calculus of fractions available for morphisms in $Z$, it is easy to check that defining $\gamma_{p(X)}:=p\left(\alpha_{X}\right)$, for any $X$ in $X$, yields the wanted natural transformation.

Since ( $y(\geq 1), y(\leq 0))$ is a stable t-structure in $y$, from [44, Proposition 1.5] we get that $(Z(\geq 1), Z(\leq 0))$ is a stable t-structure in $z$, thus proving the properties (i) and (ii) of Definition 3.1. Clearly we have Ob $z=\cup_{n \in \mathbb{Z}} \mathrm{Ob} Z(\geq n)=\cup_{n \in \mathbb{Z}} \mathrm{Ob} Z(\leq n)$ since the same relation holds for objects in $X$. Also, we have

$$
Z(\geq 1)=s \mathcal{Z}(p(X(\geq 0)))=p(s(X(\geq 0)))=p(X(\geq 1)) \subseteq p(X(\geq 0))=\mathcal{Z}(\geq 0)
$$

and similarly we get that $z(\leq-1) \subseteq z(\leq 0)$. For condition $(v)$, observe that, for any $X$ in $X$, we have

$$
\gamma_{p(X)}=p\left(\alpha_{X}\right)=p s\left(\alpha_{s^{-1} X}\right)=s \mathcal{Z}\left(\gamma_{p s^{-1}(X)}\right)=s_{\mathcal{Z}}\left(\gamma_{s_{\mathcal{Z}}^{-1}(p(X))}\right) .
$$

It remains to prove condition (vi) of Definition 3.1. Let $X$ be an object in $X(\geq 1)$ and $Y$ an object in $X(\leq 0)$. We will show that $\operatorname{Hom}_{\mathcal{Z}}\left(\gamma_{p(Y)}, p(X)\right)$ is an isomorphism. The proof that $\operatorname{Hom}_{\mathcal{Z}}\left(p(Y), \gamma_{p(X)}\right)$ is an isomorphism is analogous. Let $f: p(Y) \rightarrow p(X)$ be a morphism in $Z$, represented by a roof of the form

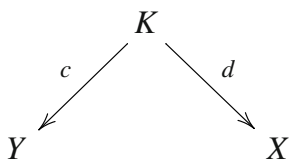

with cone $(c)$ in $y$. We want to show that $f$ admits a unique preimage under the map $\operatorname{Hom}_{\mathcal{Z}}\left(\gamma_{p(Y)}, p(X)\right)$. In order to do that, we first compose $f$ with an isomorphism and write the composition as a roof in a convenient way that will allow us to use axiom (vi) of the f-category $X$

(1) Applying the triangle functor $\sigma_{\geq 1}$ to the triangle induced by the map $c$ and using the fact that $Y$ lies in $X(\leq 0)$, it follows that $\sigma_{\geq 1} K$ lies in $Y$. Consider the composition of the natural map $\sigma_{\geq 1} K \longrightarrow K$ with $d: K \longrightarrow X$, and denote by $g: X \longrightarrow \bar{X}$ its mapping cone. It is clear that $p(g)$ is an isomorphism in $Z$. Moreover, using the fact that $\operatorname{Hom}_{\mathcal{Z}}\left(s_{\mathcal{Z}} p(Y), p(g)\right)$ and $\operatorname{Hom}_{\mathcal{Z}}(p(Y), p(g))$ are isomorphisms, the map $f$ admits a unique preimage under $\operatorname{Hom}_{z}\left(\gamma_{p(Y)}, p(X)\right)$ if and only if $\bar{f}:=p(g) \circ f$ admits a unique preimage under $\operatorname{Hom}_{z}\left(\gamma_{p(Y)}, p(\bar{X})\right)$. 
(2) Since $Y=\sigma_{\leq 0} Y, c$ factors through the natural map $K \longrightarrow \sigma_{\leq 0} K$ and the cone of $\sigma_{\leq 0} c$ is precisely $\sigma_{\leq 0}$ cone $(c)$, which lies in $y$ since $y$ is an f-subcategory of $X$. By construction of $g$, also $g \circ d$ factors through the natural map $K \longrightarrow \sigma_{\leq 0} K$ (via a map $\left.h: \sigma_{\leq 0} K \longrightarrow \bar{X}\right)$. It then can be checked that $\bar{f}: p(Y) \longrightarrow p(\bar{X})$ is equivalent to the following roof.

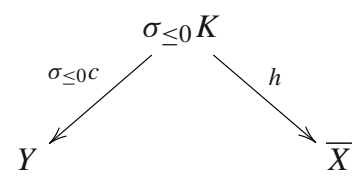

Now, from axiom (vi) of the f-category $X$ there is a unique morphism $m: s\left(\sigma_{\leq 0} K\right) \longrightarrow \bar{X}$ such that $h=m \circ \alpha_{\sigma_{\leq 0} K}$ and then, the morphism $s_{z} p(Y) \longrightarrow p(\bar{X})$ of $z$ represented by the fraction $m \circ s\left(\sigma_{\leq 0} c\right)^{-1}$ is a preimage of $\bar{f}$ by the map $\operatorname{Hom}_{z}\left(\gamma_{p(Y)}, p(\bar{X})\right)$. Using the uniqueness of $m$ and the description of morphisms in $z$ as roofs, it easily follows that this preimage is unique, finishing the proof.

We are now ready to show how an f-category over a triangulated category $\mathcal{T}$ induces f-categories over thick subcategories or over Verdier quotients. Recall that given two triangulated subcategories $\mathcal{U}$ and $\mathcal{V}$ of a triangulated category $\mathcal{T}$, one denotes by $\mathcal{U} * \mathcal{V}$ the subcategory of $\mathcal{T}$ formed by the objects $T$ such that there are objects $U$ in $\mathcal{U}, V$ in $\mathcal{V}$ and a triangle

$$
U \longrightarrow T \longrightarrow V \longrightarrow U[1]
$$

It is not always true that $\mathcal{U} * \mathcal{V}$ is a triangulated subcategory of $\mathcal{T}$. In fact, $\mathcal{U} * \mathcal{V}$ is triangulated if and only if $\operatorname{Hom}_{\mathcal{T} / \mathcal{U} \cap \mathcal{V}}(\pi(\mathcal{U}), \pi(\mathcal{V}))=0$, where $\pi: \mathcal{T} \longrightarrow \mathcal{T} /(\mathcal{U} \cap \mathcal{V})$ is the quotient functor ([45, Theorem A]).

Proposition 3.8 Let $0 \longrightarrow \mathcal{U} \stackrel{i}{\longrightarrow} \mathcal{T} \stackrel{q}{\longrightarrow} \mathcal{T} / \mathcal{U} \longrightarrow 0$ be an exact sequence of triangulated categories and $(\mathcal{X}, \theta)$ be an $f$-category over $\mathcal{T}$. Then $(\mathcal{X}, \theta)$ induces $f$-category structures over $\mathcal{U}$ and $\mathcal{T} / \mathcal{U}$.

Proof We assume without loss of generality (by Example 3.6) that $\mathcal{U}$ is a thick subcategory of $\mathcal{T}, i$ is the embedding functor (and we identify $\mathcal{U}$ with $i(\mathcal{U})$ ) and $q$ is the natural projection to the Verdier quotient.

Recall from [79, Proposition 2.2] that the f-category $(X, \theta)$ induces an f-category $y$ over $\mathcal{U}$ defined by $\mathcal{Y}=\left\{X \in X \mid \operatorname{gr}_{X}^{n}(X) \in \mathcal{U}\right.$ for all $\left.n \in \mathbb{Z}\right\}$. It is easy to check that $y$ is a thick subcategory of $\mathcal{X}$ (since $\mathcal{U}$ is a thick subcategory of $\mathcal{T}$ ) and that it is an f-subcategory of $\mathcal{X}$. Moreover, $\left(\mathcal{Y}, \theta_{\mid} \mathrm{U}\right)$ is an $\mathrm{f}$-category over $\mathcal{U}$ since we have a commutative diagram as follows, where the vertical arrows are the natural inclusions. 


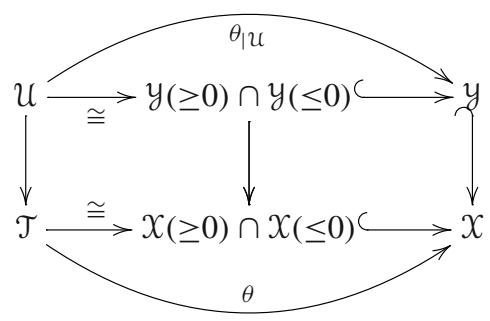

By Lemma $3.7, z:=X / \mathcal{Y}$ is an f-category. It remains to show that $X / \mathcal{Y}$ is indeed an f-category over $\mathcal{T} / \mathcal{U}$. It is clear that $\theta$ induces a functor $\bar{\theta}: \mathcal{T} / \mathcal{U} \longrightarrow X / \mathcal{Y}$ and a commutative diagram between exact sequences of triangulated categories as follows.

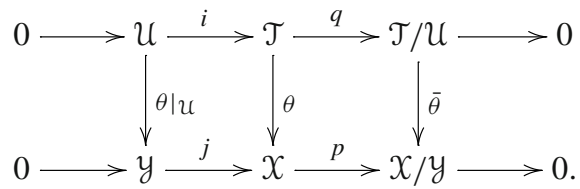

The functors $\theta$ and $\theta \mid u$ are obviously fully faithful and we claim that so is $\bar{\theta}$. Using [52, Lemma 4.7.1], it is enough to show that any map $f: Y \longrightarrow \theta(T)$ in $X$, with $Y$ in $y$ and $T$ in $\mathcal{T}$, factors through an object of

$$
\theta(\mathcal{U})=y(\geq 0) \cap y(\leq 0)=x(\geq 0) \cap x(\leq 0) \cap y=\theta(\mathcal{T}) \cap y
$$

Since $\theta(T)$ lies in $X_{(\leq 0)} \cap X(\geq 0)$, we may assume without loss of generality that $Y$ lies in $y(\leq 0)$ (this follows from the triangle in Definition 3.1(ii) and the orthogonality relation between $y(\geq 1)$ and $X(\leq 0))$. By Proposition 3.3(i), the restriction of the f-forgetful functor $\omega$ to $X(\leq 0)$ is left adjoint to the inclusion of $\mathcal{T}$ (by $\theta)$ in $X(\leq 0)$. Thus, considering the unit of the adjunction, $\eta_{Y}: Y \longrightarrow \theta \omega(Y)$, we get that $f=\theta(\tilde{f}) \circ \eta_{Y}$, where $\tilde{f}: \omega(Y) \longrightarrow T$ is the map corresponding to $f$ under the isomorphism $\operatorname{Hom}_{\mathcal{X}}(Y, \theta(T)) \cong \operatorname{Hom}_{\mathcal{T}}(\omega(Y), T)$. We now show that $\omega(Y)$ lies in $\mathcal{U}$. We do this by induction on the graded length of $Y$, i.e. on $n \geq 0$ such that $Y$ lies in $X(\geq-n) \cap X(\leq 0)$ (such $n$ always exists by Definition 3.1(iii) and by our assumption that $Y$ lies in $X(\leq 0)$ ). If $Y$ lies in $X(\geq 0)$, then $Y \cong \theta \operatorname{gr}^{0} Y$ and $\omega(Y)=\theta^{-1}(Y)=\operatorname{gr}^{0}(Y)$ lies in $\mathcal{U}$, by definition of $\mathcal{Y}$. Suppose now that the result is valid for objects with graded length $n-1$ and let $Y$ lie in $y(\geq-n)$. Then there is a triangle

$$
Y=\sigma_{\geq-n} Y \longrightarrow \sigma_{\geq-n+1} Y \longrightarrow s^{n} \theta \operatorname{gr}^{-\mathrm{n}+1}(Y) \longrightarrow\left(\sigma_{\geq-n} Y\right)[1]
$$

Applying the triangle functor $\omega$ to it, since $\omega s^{n} \theta \mathrm{gr}^{-\mathrm{n}+1}(Y) \cong \mathrm{gr}^{-\mathrm{n}+1}(Y)$ lies in $\mathcal{U}$ and, by induction hypothesis, so does $\omega \sigma_{\geq-n+1} Y$, it follows that $\omega(Y)$ lies in $\mathcal{U}$, as wanted.

It remains to show that the essential image of $\bar{\theta}$ is $Z(\geq 0) \cap Z(\leq 0)$. By the commutativity of (3.2) and since $\bar{\theta}$ is fully faithful, it suffices to prove that $Z(\geq 0) \cap Z(\leq 0)=$ $p(X(\geq 0) \cap X(\leq 0))$. We first show that $z(\geq 0) \cong(X(\geq 0) * y) / y=p(X(\geq 0) * y)$ (dual arguments also show that $\mathcal{Z}(\leq 0) \cong(y * X(\leq 0)) / y=p(y * X(\leq 0)))$. Note that $x(\geq 0) \cap y=y(\geq 0)$. Using [45, Theorem A], it is then enough to prove that $\operatorname{Hom}_{X / Y(\geq 0)}(\pi(X(\geq 0)), \pi(y))=0$, where $\pi: X \longrightarrow X / Y(\geq 0)$ is the Verdier quotient functor. Consider an element in $\operatorname{Hom}_{X / Y(\geq 0)}(\pi(X), \pi(Y))$, with $X$ in $X(\geq 0)$ and $Y$ in $y$, represented by a roof 


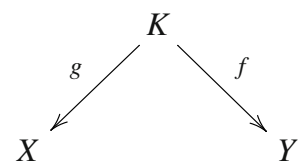

with $f \in \operatorname{Hom}_{X}(K, Y), g \in \operatorname{Hom}_{X}(K, X)$ and cone(g) in $y(\geq 0)$. Since both $X$ and cone(g) lie in $X(\geq 0)$, it follows that also $K$ lies in $X(\geq 0)$. Hence, it follows that $f$ must factor through the natural map $\sigma_{\geq 0} Y \longrightarrow Y$. In particular, the roof is equivalent to the zero morphism in $X / Y(\geq 0)$, as wanted. Thus we may rewrite the intersection $Z(\leq 0) \cap Z(\geq 0)$ as follows

$$
\mathcal{Z}(\leq 0) \cap \mathcal{Z}(\geq 0)=p(y * X(\leq 0)) \cap p(X(\geq 0) * y)=p((y * X(\leq 0)) \cap(X(\geq 0) * y)),
$$

where the last equality follows from [45, Lemma 2.4(i)(a)]. Finally, we finish the proof by showing that the last term above equals $p(X(\leq 0) \cap X(\geq 0))$. Observe first that given an object $X$ in $X(\geq 0) * y$, it follows that $\sigma_{\leq-1} X$ lies in $y$. In fact, by the assumption on $X$ there is a triangle

$$
X^{\prime} \stackrel{f}{\longrightarrow} X \longrightarrow Y \longrightarrow X^{\prime}[1]
$$

with $X^{\prime}$ in $X(\geq 0)$ and $Y$ in $Y$. Applying to it the triangle functor $\sigma_{\leq-1}$, since $\sigma_{\leq-1} X^{\prime}=0$ we get that $\sigma_{\leq-1} X \cong \sigma_{\leq-1} Y$. Since $\mathcal{Y}$ is an f-subcategory of $X$, it then follows that $\sigma_{\leq-1} X$ lies in $Y$. Analogously, one can show that given $X$ in $y * X(\leq 0), \sigma_{\geq 1} X$ lies in $y$. Hence for any object $X$ in the intersection $(y * X(\leq 0)) \cap(X(\geq 0) * y)$, both $\sigma_{\leq-1} X$ and $\sigma_{\geq 1} X$ lie in $y$ and, thus, $p(X) \cong p\left(\theta \operatorname{gr}^{0}(X)\right)$, showing that $p((Y * X(\leq 0)) \cap(X(\geq 0) * y))$ is contained in $p(X(\leq 0) \cap X(\geq 0))$. Since the other inclusion is trivial (because $X(\leq 0) \cap X(\geq 0) \subset$ $(y * X(\leq 0)) \cap(X(\geq 0) * y))$, we have finished the proof.

Corollary 3.9 Let $0 \longrightarrow \mathcal{U} \stackrel{i}{\longrightarrow} \mathcal{T} \stackrel{q}{\longrightarrow} \mathcal{T} / \mathcal{U} \longrightarrow 0$ be an exact sequence of triangulated categories and $(X, \theta)$ be an $f$-category over $\mathcal{T}$. Then the functors $i$ and $q$ admit f-liftings for suitable choices of f-categories over $\mathcal{U}$ and $\mathcal{T} / \mathcal{U}$.

Proof By construction of the f-categories $\left(y, \theta_{\mid} u\right)$ and $(\mathcal{Z}, \bar{\theta})$ in the proof of Proposition 3.8, we get that $i$ and $j$ admit f-lifitings, namely the f-functors $j$ and $p$ in the diagram (3.2) above.

Remark 3.10 Recall from [61, Definition 2.4] that a morphism of triangles $(a, b, c)$ is said to be middling good if it can be completed to a commutative $3 \times 3$ diagram (in the sense of [16, Lemma 1.1.11]) in which all rows and all columns are triangles. In [74], the following extra axiom for $\mathrm{f}$-categories is proposed.

(fcat7) For any morphism $f: X \longrightarrow Y$ in $X$, the triple $\Delta_{f}:=\left(\alpha_{\sigma_{\geq 1(Y)}} \circ \sigma_{\geq 1}(f), \alpha_{Y} \circ\right.$ $\left.f, \alpha_{\sigma_{\leq 0(Y)}} \circ \sigma_{\leq 0}(f)\right)$ is a middling good morphism of triangles.

Although we have not made use of this axiom so far, we will implicitly make use of it in the next subsection (see Remark 3.12(ii) and the Appendix to this paper). At this point it is worth noting the following facts.

- Filtered derived categories, as discussed in Example 3.2 satisfy axiom (vii). This is proved in [74, Lemma 7.4].

- In the context of Lemma 3.7, if $X$ satisfies axiom (fcat7), then so does $Z:=X / Y$. Note that if a morphism of triangles is middling good, then so is its composition with an isomorphism of triangles. Hence, given $f: X \longrightarrow Y$ in $Z$, we may assume without loss 
of generality that $f=p\left(f^{\prime}\right)$, where $p$ is the Verdier quotient functor. Since $X$ satisfies axiom (vii), the morphism $\Delta_{f^{\prime}}$ is middling good. As a consequence $p\left(\Delta_{f^{\prime}}\right)$ is middling good. Finally, using the t-exactness of $p$ and the compatibility of $p$ with the functor $s$ and the natural transformations $\alpha$ and $\gamma$, we conclude that $p\left(\Delta_{f^{\prime}}\right)=\Delta_{p\left(f^{\prime}\right)}$ (see the proof of Lemma 3.7 for notation and details).

From now on, we will assume f-categories to satisfy this new axiom.

\subsection{Realisation functors and their properties}

We are now ready to build realisation functors. Let $\mathcal{T}$ be a triangulated category and $\mathbb{T}=\left(\mathbb{T}^{\leq 0}, \mathbb{T}^{\geq 0}\right)$ a t-structure in $\mathcal{T}$. Suppose that $(\mathcal{X}, \theta)$ is an $\mathrm{f}$-category over $\mathcal{T}$. By Proposition 3.3 , there is a t-structure $\mathbb{X}$ in $\mathcal{X}$ defined by

$$
\begin{gathered}
\mathbb{X}^{\leq 0}=\left\{X \in X \mid \operatorname{gr}_{X}^{n}(X) \in \mathbb{T}^{\leq n} \text { for all } n \in \mathbb{Z}\right\} \text { and } \\
\quad \mathbb{X}^{\geq 0}=\left\{X \in X \mid \operatorname{gr}_{X}^{n}(X) \in \mathbb{T}^{\geq n} \text { for all } n \in \mathbb{Z}\right\} ;
\end{gathered}
$$

whose heart $\mathcal{H}(\mathbb{X})$ is equivalent to $\mathrm{C}^{b}(\mathcal{H}(\mathbb{T}))$. Let $G: \mathrm{C}^{b}(\mathcal{H}(\mathbb{T})) \longrightarrow \mathcal{H}(\mathbb{X})$ denote the inverse of that equivalence (described in Remark 3.4). The realisation functor of $\mathbb{T}$ with respect to the f-category $(\mathcal{X}, \theta)$ is then obtained as follows. Moreover, we collect the first properties of the functor real $\left.\right|_{\mathbb{T}} ^{x}$. These were first proved in [16] and restated in a more general setting in [79]. Our statement differs to that in [79] only on the class of triangulated categories we consider-see Remark 3.12(i). We also include a sketch for the proof of the theorem for the convenience of the reader.

Theorem 3.11 [16, Section 3.1] [15, Appendix] [79, Theorem 1.1] Let $\mathcal{T}$ be a triangulated category and $(X, \theta)$ an $f$-category over $\mathcal{T}$ with f-forgetful functor $\omega: X \longrightarrow \mathcal{T}$. Let $\mathbb{T}$ be a t-structure in $\mathcal{T}, \mathbb{X}$ the corresponding induced $t$-structure on $X$ and $G: C^{b}(\mathcal{H}(\mathbb{T})) \longrightarrow$ $\mathcal{H}(\mathbb{X})$ the exact equivalence of abelian categories described in the paragraph above. Let $Q: \mathrm{C}^{b}(\mathcal{H}(\mathbb{T})) \longrightarrow \mathrm{D}^{b}(\mathcal{H}(\mathbb{T}))$ be the natural localisation functor. Then there is a unique functor, called the realisation functor of $\mathbb{T}$ with respect to $(X, \theta)$, real $\left.\right|_{\mathbb{T}} ^{X}: D^{b}(\mathcal{H}(\mathbb{T})) \longrightarrow \mathcal{T}$ such that the following diagram naturally commutes

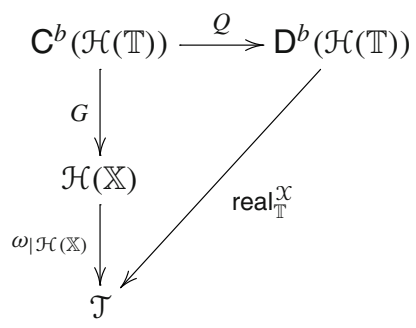

Furthermore, real $\mathbb{T}_{\mathbb{T}}^{\chi}: \mathrm{D}^{b}(\mathcal{H}(\mathbb{T})) \longrightarrow \mathcal{T}$ is a triangle functor and satisfies the following properties.

(i) $\mathrm{H}_{0}^{i} \cong \mathrm{H}_{\mathbb{T}}^{i} \circ$ real $\mathbb{T}_{\mathbb{T}}^{\chi}$, for all $i \in \mathbb{Z}$. In particular, real $\mathrm{T}_{\mathbb{T}}^{X}$ acts as the identity functor on $\mathcal{H}(\mathbb{T})$ and it is t-exact with respect to the standard $t$-structure in $\mathrm{D}^{b}(\mathcal{H}(\mathbb{T}))$ and $\mathbb{T}$ in $\mathcal{T}$.

(ii) The functor real $\left.\right|_{\mathbb{T}} ^{X}$ induces isomorphisms $\operatorname{Hom}_{\mathbb{D}^{b}(\mathcal{H}(\mathbb{T}))}(X, Y[n]) \cong \operatorname{Hom}_{\mathcal{T}}(X, Y[n])$ for any $X$ and $Y$ in $\mathcal{H}(\mathbb{T})$ and for $n \leq 1$.

(iii) The following statements are equivalent. 
(a) The functor real $\left.\right|_{\mathbb{T}} ^{x}$ is fully faithful;

(b) The functor real ${ }_{\mathbb{T}}^{x}$ induces isomorphisms $\operatorname{Hom}_{\mathrm{D}^{b}(\mathcal{H}(\mathbb{T}))}(X, Y[n]) \cong \operatorname{Hom}_{\mathcal{T}}(X, Y[n])$, for all $n \geq 2$ and for all $X$ and $Y$ in $\mathcal{H}(\mathbb{T})$.

(c) (Ef) Given objects $X$ and $Y$ in $\mathcal{H}(\mathbb{T}), n \geq 2$ and a morphism $f: X \longrightarrow Y[n]$ in $\mathcal{T}$, there is an object $Z$ in $\mathcal{H}(\mathbb{T})$ and an epimorphism in $\mathcal{H}(\mathbb{T}), g: Z \longrightarrow X$, such that $f g=0$.

(d) (CoEf): Given objects $X$ and $Y$ in $\mathcal{H}(\mathbb{T}), n \geq 2$ and a morphism $f: X \longrightarrow Y[n]$ in $\mathcal{T}$, there is an object $Z$ in $\mathcal{H}(\mathbb{T})$ and a monomorphism in $\mathcal{H}(\mathbb{T}), g: Y \longrightarrow Z$, such that $g[n] f=0$.

(iv) The essential image of real $\mathbf{I}_{\mathbb{T}}^{x}$ is contained in

$$
\mathcal{T}^{b(\mathbb{T})}:=\bigcup_{n, m \in \mathbb{Z}} \mathbb{T}^{\leq n} \cap \mathbb{T}^{\geq m}
$$

and it coincides with it whenever real $\mathbb{T}_{\mathbb{T}}^{X}$ is fully faithful.

Proof For the existence of the functor real $\left.\right|_{\mathbb{T}} ^{x}$ and for property (i) we refer to [15, Appendix A.5, A.6]. Property (ii) is clear for $n \leq 0$. For $n=1$, the statement boils down to show that the Yoneda extension group $\operatorname{Ext}_{\mathcal{H}(\mathbb{T})}^{1}(X, Y)$ in $\mathcal{H}(\mathbb{T})$ coincides with $\operatorname{Hom}_{\mathcal{T}}(X, Y[1])$, which is a well-known fact about hearts of t-structures (see [16, Remark 3.1.17(ii)]).

For part (iii), we prove in detail the most delicate implication: (c) $\Longrightarrow$ (b) (the implication $(\mathrm{d}) \Longrightarrow(b)$ is analogous). Assuming the condition (Ef) we show the isomorphism in (b) by induction on $n \geq 1$ (for $n=1$, (b) holds by statement (ii)). Let $X$ and $Y$ be objects in $\mathcal{H}(\mathbb{T}), n \geq 2$ and consider the induced map real $\mathbb{T}_{\mathbb{T}}^{X}(X, Y[n]): \operatorname{Hom}_{\mathrm{D}^{b}(\mathcal{H}(\mathbb{T}))}(X, Y[n]) \longrightarrow$ $\operatorname{Hom}_{\mathcal{T}}(X, Y[n])$. First we prove surjectivity. Let $f: X \longrightarrow Y[n]$ be a map in $\mathcal{T}$. By assumption there is an epimorphism $g: Z \longrightarrow X$ in $\mathcal{H}(\mathbb{T})$ such that $f \circ g=0$ in $\mathcal{T}$. Let $K$ be the kernel of $g$ in $\mathcal{H}(\mathbb{T})$ and consider the triangle induced by $g$ in $\mathcal{T}$ :

$$
K \longrightarrow Z \stackrel{g}{\longrightarrow} X \stackrel{h}{\longrightarrow} K[1] .
$$

Since $g \circ f=0$, there is $u: K[1] \longrightarrow Y[n]$ such that $u \circ h=f$. By induction hypothesis, there is $u^{\prime}$ in $\operatorname{Hom}_{\mathrm{D}^{b}(\mathcal{H}(\mathbb{T}))}(K[1], Y[i])$ such that real $\left.\right|_{\mathbb{T}} ^{X}\left(u^{\prime}\right)=u$. By (ii), there is also $h^{\prime}$ in $\operatorname{Hom}_{\mathrm{D}^{b}(\mathcal{H}(\mathbb{T}))}(X, K[1])$ such that real $\mathbb{T}_{\mathbb{T}}^{X}\left(h^{\prime}\right)=h$ and, thus, we have that real $_{\mathbb{T}}^{X}\left(u^{\prime} \circ h^{\prime}\right)=u \circ h=f$. To prove the injectivity of real $\left.\right|_{\mathbb{T}} ^{X}(X, Y[n])$, let $\alpha$ be an element in $\operatorname{Hom}_{\mathrm{D}^{b}(\mathcal{H}(\mathbb{T}))}(X, Y[n])$ such that real $\mathbb{T}_{\mathbb{T}}^{X}(\alpha)=0$. Since $\alpha$ can be thought of as an Yoneda extension (of degree $n$ ) between $X$ and $Y$, it represents an exact sequence in $\mathcal{H}(\mathbb{T})$ of the form

$$
0 \longrightarrow Y \longrightarrow A_{1} \longrightarrow A_{2} \longrightarrow \cdots \longrightarrow A_{n} \stackrel{\beta}{\longrightarrow} X \longrightarrow 0 .
$$

It is easy to check that $\alpha \circ \beta=0$. Let $L$ denote the kernel of $\beta$ and consider the triangle in $\mathrm{D}^{b}(\mathcal{H}(\mathbb{T}))$ :

$$
A_{n} \stackrel{\beta}{\longrightarrow} X \stackrel{\gamma}{\longrightarrow} L[1] \stackrel{\rho[1]}{\longrightarrow} A_{n}[1]
$$

Then there is $\delta: L[1] \longrightarrow Y[n]$ such that $\alpha=\delta \circ \gamma$. Now, we have that $0=\operatorname{real}_{\mathbb{T}}^{x}(\alpha)=$ $\operatorname{real}_{\mathbb{T}}^{x}(\delta) \circ \operatorname{real}_{\mathbb{T}}^{x}(\gamma)$ and, hence, there is a map $\epsilon: A_{n}[1] \longrightarrow Y[n]$ in $\mathcal{T}$ such that 
real $_{\mathbb{T}}^{x}(\delta)=\epsilon$ real $_{\mathbb{T}}^{x}(\rho[1])$. Since real $\left.\right|_{\mathbb{T}} ^{x}\left(A_{i}[1], Y[i]\right)$ is surjective, by induction hypothesis, it follows that there is a map $\epsilon^{\prime}: A_{i}[1] \longrightarrow Y[i]$ in $\mathrm{D}^{b}(\mathcal{H}(\mathbb{T}))$ such that real $\left.\right|_{\mathbb{T}} ^{x}\left(\epsilon^{\prime}\right)=\epsilon$. Now, real $\left.\right|_{\mathbb{T}} ^{X}\left(\epsilon^{\prime} \circ \rho[1]\right)=$ real $_{\mathbb{T}}^{X}(\delta)$ which, since real $\left.\right|_{\mathbb{T}} ^{X}(L[1], Y[i])$ is injective by induction hypothesis, implies that $\epsilon^{\prime} \circ \rho[1]=\delta$. Hence, we have $\alpha=\delta \circ \gamma=\epsilon^{\prime} \circ \rho[1] \circ \gamma=0$ since $\rho[1] \circ \gamma=0$.

With regards to the remaining implications of (iii): it is clear that (a) implies (b) and the converse follows from a dévissage argument (see our proof of Theorem 6.9 for such an argument). The fact that (a) implies (c) or (d) can easily be observed from properties of Yoneda extensions (in fact the proof that (a) implies (c) is essentially contained in the above paragraph). Finally, regarding property (iv), since real $\left.\right|_{\mathbb{T}} ^{x}$ is t-exact and the standard t-structure in $D^{b}(\mathcal{H}(\mathbb{T}))$ is bounded, it follows easily that the $\operatorname{Im}\left(\right.$ real $\left.\left.\right|_{\mathbb{T}} ^{x}\right)$ is contained in $\mathcal{T}^{b(\mathbb{T})}$. If real $\mathbb{T}_{\mathbb{T}}^{\chi}$ is fully faithful, it can be proved by induction on $l=b-a$ (with $b \geq a$ ) that $\mathbb{T}^{\leq b} \cap \mathbb{T}^{\geq a}$ lies in its essential image (see the proof of [16, Proposition 3.1.16]).

Remark 3.12 (i) In [79] the above result is stated for triangulated subcategories of the derived category of an abelian category. This restriction is only to ensure that the triangulated category considered admits an $f$-enhancement, but the arguments carry through in the more general setting here considered.

(ii) Although the claim that real $\mathbb{T}_{\mathbb{T}}^{X}$ is a triangle functor is implicit in [15, Appendix], the only proof of this fact that the authors are aware of is due to E. Cabezuelo Fernández and O. Schnürer and it makes use of the axiom (vii) in Remark 3.10 (see the Appendix to this paper for details)

One further property of realisation functors that is particularly useful in our applications is that they behave naturally in certain contexts. The following theorem was presented in [15] without proof.

Theorem 3.13 [15, Lemma A7.1] Let $(X, \theta)$ and $(y, \eta)$ be $f$-categories over triangulated categories $\mathcal{T}$ and $\mathcal{U}$, respectively, and let $\phi: \mathcal{T} \longrightarrow \mathcal{U}$ be a triangle functor. Suppose that $\phi$ is $t$-exact with respect to $t$-structures $\mathbb{T}$ and $\mathbb{U}$ in $\mathcal{T}$ and $\mathcal{U}$, respectively. If $\phi$ admits an $f$-lifting, then there is a commutative diagram

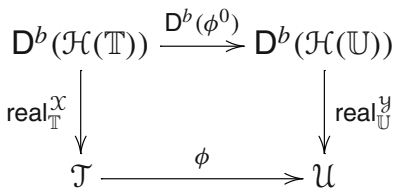

where $\mathrm{D}^{b}\left(\phi^{0}\right)$ is the derived functor of the exact functor $\phi^{0}: \mathcal{H}(\mathbb{T}) \longrightarrow \mathcal{H}(\mathbb{U})$, induced by $\phi \mid \mathcal{H}(\mathbb{T})$.

Proof Let $\Phi: X \longrightarrow y$ be an f-lifting of $\phi$ and let $\mathbb{X}$ and $\mathbb{Y}$ be the t-structures in $X$ and $y$ compatible with $\mathbb{T}$ and $\mathbb{U}$ as in Proposition 3.3(ii). Since $\Phi$ is an f-lifting of $\phi$ (and, in particular, an f-functor), we have

$$
\begin{aligned}
\phi\left(\operatorname{gr}_{X}^{n}(X)\right) & =\phi\left(\theta^{-1} s^{-n} \sigma_{\leq n} \sigma_{\geq n}(X)\right) \\
& \cong \eta^{-1} \Phi \mid x(\geq 0) \cap X(\leq 0) s^{-n} \sigma_{\leq n} \sigma_{\geq n}(X) \\
& \cong \eta^{-1} t^{-n} \Phi\left(\sigma_{\leq n} \sigma_{\geq n}(X)\right) \\
& \cong \eta^{-1} t^{-n} \sigma_{\leq n} \sigma_{\geq n} \Phi(X) \\
& \cong \operatorname{gr}_{y}^{n}(\Phi(X)) .
\end{aligned}
$$


Using this fact, since $\phi$ is t-exact (with respect to $\mathbb{T}$ and $\mathbb{U}$ ), we get that $\Phi$ is also t-exact (with respect to $\mathbb{X}$ and $\mathbb{Y}$ ), inducing an exact functor $\Phi^{0}: \mathcal{H}(\mathbb{X}) \longrightarrow \mathcal{H}(\mathbb{Y})$. This yields the following diagram of functors.

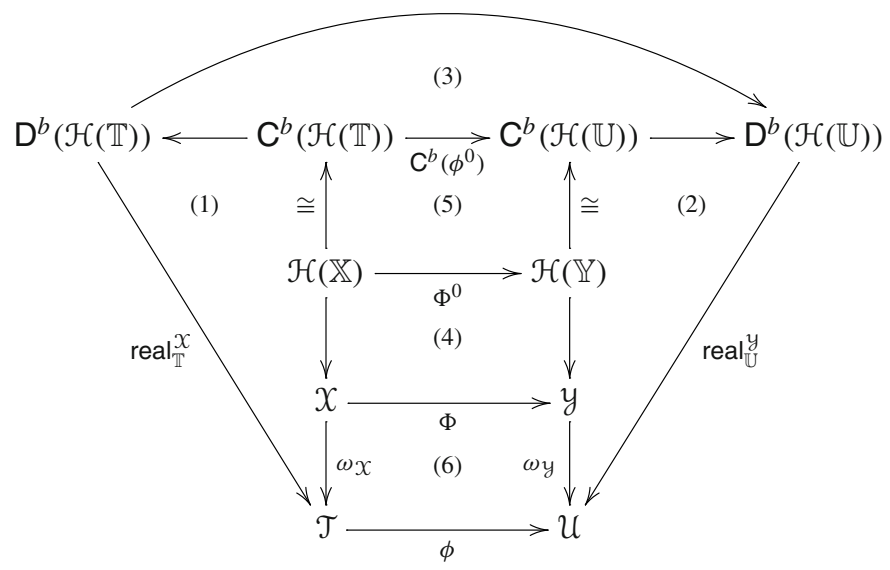

In order to prove the theorem, it is enough to check the commutativity of all the internal diagrams. Diagrams (1), (2), (3) and (4) commute by construction of the functors involved. Diagram (5) commutes using again above property that $\phi \operatorname{gr}_{X}^{n} \cong \operatorname{gr}_{y}^{n} \Phi$ and Remark 3.4.

Finally, let us prove in detail the commutativity of diagram (6). We first show that (6) naturally commutes for objects in $X(\leq 0)$, i.e. that there is a natural equivalence $\mu^{0}: \omega y_{\mid y(\leq 0)} \Phi_{\mid X(\leq 0)} \longrightarrow \phi \omega x_{\mid X}(\leq 0)$. To simplify the notation we write the upperscript $\leq 0$ to denote the restriction of the functors to $X(\leq 0)$ or to $y(\leq 0)$ (depending on the domain of the functor). Consider the unit of the adjunction $\left(\omega_{\mathcal{X}}^{\leq 0}, \theta\right)$ and denote it by $\delta: \operatorname{Id} \mathcal{X}_{(\leq 0)} \longrightarrow \theta \omega_{\bar{X}}^{\leq 0}$. We define $\mu^{0}$ as the following natural composition

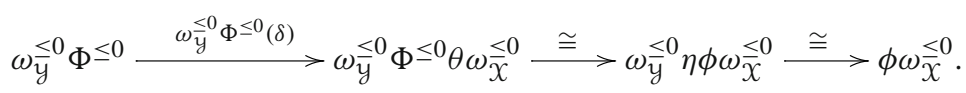

Note that we use the fact that $\Phi$ is an f-lifting of $\phi$ in order to get a natural equivalence $\Phi^{\leq 0} \theta \cong \eta \phi$. We also use that $\omega_{y}^{\leq 0}$ is a left inverse to $\eta$. Consider now the subcategory of $X_{(\leq 0)}$ formed by all the objects $X$ such that $\mu_{X}^{0}$ is an isomorphism. It is easy to see that this subcategory is triangulated and it contains $s^{n}(X(\leq 0) \cap X(\geq 0))=s^{n} \theta(\mathcal{T})$ for any $n \leq 0$. Since every object in $X(\leq 0)$ can be obtained as a finite extension of such objects, it follows that $\mu^{0}$ is a natural equivalence. Now, given $n \geq 0$, we define natural transformations

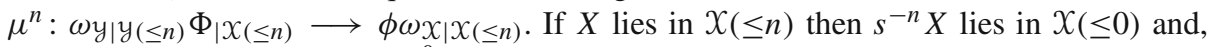
hence, we may define $\mu^{n}:=\mu^{0} s^{-n}$. It is clear that $\mu^{n}$ is also a natural equivalence. Thus, we have a family of natural equivalences $\left(\mu^{n}\right)_{n \geq 0}$. It follows from Definition 3.1(iii) and (iv) that in order to define a natural equivalence $\mu: \omega y \Phi \longrightarrow \phi \omega x$, it is enough to show that, for any $m>n$ and for any $X$ in $X(\leq n), \mu_{X}^{n}$ is naturally isomorphic to $\mu_{X}^{m}$ (note that $\left.\mu^{m}=\mu^{n} s^{n-m}\right)$. Let $X$ lie in $X(\leq n)$ and consider the map

$$
\mu_{s^{n-m} X}^{n}: \omega y \Phi\left(s^{n-m} X\right) \longrightarrow \phi \omega x\left(s^{n-m} X\right)
$$

Using property (iii) in Definition 3.1, we define the following composition of natural morphisms

$$
\alpha_{X}^{[n, m]}: s^{n-m} X \stackrel{\alpha_{s^{n-m} X}}{\longrightarrow} s^{n-m+1} X \stackrel{\alpha_{s^{n-m+1} X}}{\longrightarrow} \cdots \stackrel{\alpha_{s^{-1} X}}{\longrightarrow} X .
$$


By the naturality of $\alpha$, we get a commutative diagram as follows

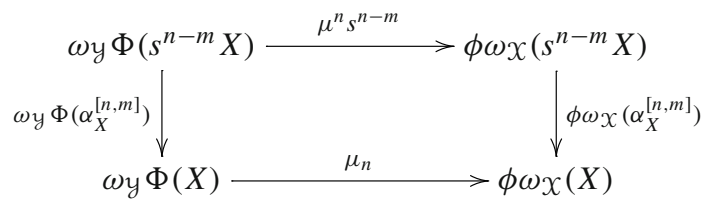

Since $\Phi$ is an f-functor, $\Phi\left(s^{n-m} X\right)$ is naturally isomorphic to $t^{n-1} \Phi(X)$ and $\Phi\left(\alpha_{X}^{[n, m]}\right)=\beta_{\Phi(X)}^{[n, m]}$. By Proposition 3.3(i) it follows that the vertical maps are isomorphisms, as wanted.

\subsection{Examples of realisation functors}

We begin with the simplest realisation functor: the one associated to the standard t-structure in a derived category and with respect to the filtered derived category.

Proposition 3.14 Let $\mathscr{A}$ be an abelian category. Then the realisation functor associated to the standard t-structure in $\mathrm{D}(\mathscr{A})$ with respect to the filtered derived category of $\mathscr{A}$ is naturally equivalent to the inclusion functor of $\mathrm{D}^{b}(\mathscr{A})$ in $\mathrm{D}(\mathscr{A})$.

Proof Going through the construction of the realisation functor for the standard t-structure, we show that it acts as the identity both on objects and on morphisms. From Proposition 3.3 (ii), there is a t-structure in the filtered derived category $\operatorname{DF}(\mathscr{A})$ compatible with the standard $\mathrm{t}$-structure in $\mathrm{D}(\mathscr{A})$, whose heart is

$$
\mathscr{A} \mathrm{F}:=\left\{(X, F) \in \mathrm{DF}(\mathscr{A}) \mid \operatorname{gr}_{F}^{i}(X) \in \mathscr{A}[-i] \forall i \in \mathbb{Z}\right\}
$$

and the equivalence between $\mathscr{A} \mathrm{F}$ and $\mathrm{C}^{b}(\mathscr{A})$ (as in Remark 3.4) is given by assigning to $(X, F)$ in $\mathscr{A} \mathrm{F}$ the complex $\left(\operatorname{gr}_{F}^{i}(X), d^{i}\right)$, where $d^{i}: \operatorname{gr}_{F}^{i}(X) \longrightarrow \operatorname{gr}_{F}^{i+1}(X)$ is defined by the canonical triangle in $\mathrm{D}^{b}(\mathscr{A})$

$$
\operatorname{gr}_{F}^{i}(X)[-1] \stackrel{d^{i}}{\longrightarrow} \operatorname{gr}_{F}^{i+1}(X) \longrightarrow F_{i} X / F_{i+2} X \longrightarrow \operatorname{gr}_{F}^{i}(X)
$$

Note that this makes sense since the map $d^{i}$ in the above triangle is indeed a map in $\mathscr{A}[-i-1]$, by definition of $\mathscr{A} \mathrm{F}$. In order to compute the realisation functor, one needs to describe an inverse of this equivalence of abelian categories. Given a complex $Y=\left(Y^{i}, d^{i}\right)$ in $\mathrm{C}^{b}(\mathscr{A})$ consider a filtration on $Y$ defined by the stupid truncations, i.e. for any integer $n$ define

$$
F_{n} Y=\left(\cdots \longrightarrow 0 \longrightarrow Y^{n} \stackrel{d^{n}}{\longrightarrow} Y^{n+1} \stackrel{d^{n+1}}{\longrightarrow} Y^{n+2} \stackrel{d^{n+2}}{\longrightarrow} \cdots\right),
$$

It is easy to see that, in fact, the object $(Y, F)$ belongs to $\mathscr{A} \mathrm{F}$. This assignment clearly gives rise to a functor $G: \mathrm{C}^{b}(\mathscr{A}) \longrightarrow \mathscr{A} \mathrm{F}$ which can easily be checked to be the wanted inverse functor. Consider now the composition $\mathrm{C}^{b}(\mathscr{A}) \longrightarrow \mathscr{A} \mathrm{F} \longrightarrow \mathrm{DF}(\mathscr{A}) \longrightarrow \mathrm{D}(\mathscr{A})$ of $G$ and the forgetful functor $\omega: \operatorname{DF}(\mathscr{A}) \longrightarrow \mathrm{D}(\mathscr{A})$. It is clear that this composition sends a complex $Y$ to itself as an object of the derived category-and similarly for morphisms. Hence, the realisation functor, being the universal functor induced by the localisation of $\mathrm{C}^{b}(\mathscr{A})$ at the quasi-isomorphisms, is naturally equivalent to the inclusion functor of $\mathrm{D}^{b}(\mathscr{A})$ in $\mathrm{D}(\mathscr{A})$.

In the above proposition, if we restrict the codomain to $D^{b}(\mathscr{A})$, the realisation functor is then naturally equivalent to $\operatorname{Id}_{\mathrm{D}^{b}(\mathscr{A})}$. Throughout the paper we will restrict the codomain 
of the realisation functor from unbounded derived categories to bounded ones whenever possible and convenient without further mention.

A recurrent problem when dealing with realisation functors is that, as they are defined, their domain is a bounded (rather than unbounded) derived category. In order to also discuss functors defined in unbounded derived categories, we need the following notion.

Definition 3.15 Let $\mathscr{A}$ and $\mathscr{B}$ be abelian categories. An equivalence $\Phi: \mathrm{D}(\mathscr{A}) \longrightarrow \mathrm{D}(\mathscr{B})$ is said to be restrictable if, by restriction, it induces an equivalence $\phi: \mathrm{D}^{b}(\mathscr{A}) \longrightarrow \mathrm{D}^{b}(\mathscr{B})$. In this case we also say that $\phi$ is extendable. In other words, the equivalences $\Phi$ and $\phi$ are, respectively, restrictable or extendable if there is a commutative diagram as follows, where the vertical arrows are the natural inclusions.

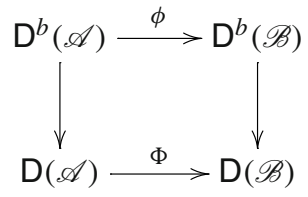

Example 3.16 (i) Let $\mathscr{A}$ and $\mathscr{B}$ be abelian categories and $\Phi: \mathrm{D}(\mathscr{A}) \longrightarrow \mathrm{D}(\mathscr{B})$ a triangle equivalence. Then $\Phi$ is restrictable if and only if $\mathrm{D}(\mathscr{B})^{b(\mathbb{T})}=\mathrm{D}^{b}(\mathscr{B})$, where $\mathbb{T}:=$ $\left(\Phi\left(\mathbb{D}^{\leq 0}\right), \Phi\left(\mathbb{D}^{\geq 0}\right)\right.$ ) (for further equivalent conditions on the t-structure, see Lemma 4.14). In fact, since $\Phi_{\mid \mathrm{D}^{b}(\mathscr{A})}$ is fully faithful, the objects in $\operatorname{Im} \Phi_{\mid \mathrm{D}^{b}(\mathscr{A})}$ are precisely those which can be obtained as finite extensions in $\mathrm{D}(\mathscr{B})$ of shifts of objects in $\mathcal{H}(\mathbb{T})=$ $\Phi(\mathscr{A})$ - and this is precisely the subcategory $\mathrm{D}(\mathscr{B})^{b(\mathbb{T})}$.

(ii) Equivalences of standard type between bounded derived categories of rings are extendable [46].

The next proposition shows that equivalences of unbounded or bounded derived categories do not differ much from suitably chosen realisation functors. By this we mean that the difference between a derived equivalence and our choice of realisation functor is a trivial derived equivalence, i.e. the derived functor of an exact equivalence of abelian categories.

Proposition 3.17 Let $\mathscr{A}$ and $\mathscr{B}$ be abelian categories. The following statements hold.

(i) Let $\phi: \mathrm{D}^{b}(\mathscr{A}) \longrightarrow \mathrm{D}^{b}(\mathscr{B})$ be a triangle equivalence and let $\mathbb{T}$ be the $t$-structure $\left(\phi\left(\mathbb{D}_{\mathscr{A}}^{\leq 0}\right), \phi\left(\mathbb{D}_{\mathscr{A}}^{\geq 0}\right)\right)$ in $\mathrm{D}^{b}(\mathscr{B})$. Then there is an $f$-category $(X, \theta)$ over $\mathrm{D}^{b}(\mathscr{B})$ such that $\phi \cong \operatorname{real}_{\mathbb{T}}^{x} \circ \mathrm{D}^{b}\left(\phi^{0}\right)$, for the exact equivalence of abelian categories $\phi^{0}: \mathscr{A} \longrightarrow \phi(\mathscr{A})$, induced by $\phi$.

(ii) Let $\Phi: \mathrm{D}(\mathscr{A}) \longrightarrow \mathrm{D}(\mathscr{B})$ be a triangle equivalence and let $\mathbb{T}$ be the $t$-structure $\left(\phi\left(\mathbb{D}_{\mathscr{A}}^{\leq 0}\right), \phi\left(\mathbb{D}_{\mathscr{A}}^{\geq 0}\right)\right)$ in $\mathrm{D}(\mathscr{B})$. Then there is an $f$-category $(X, \theta)$ over $\mathrm{D}(\mathscr{B})$ such that $\Phi^{b}:=\Phi_{\mid \mathrm{D}^{b}(\mathscr{A})} \cong$ real $_{\mathbb{T}}^{X} \circ \mathrm{D}^{b}\left(\Phi^{0}\right)$, for the exact equivalence of abelian categories $\Phi^{0}: \mathscr{A} \longrightarrow \Phi(\mathscr{A})$, induced by $\Phi$. In particular, if $\operatorname{Im}\left(\right.$ real $\left._{\mathbb{T}}^{x}\right)=\mathrm{D}(\mathscr{B})^{b(\mathbb{T})}=\mathrm{D}^{b}(\mathscr{B})$, then $\Phi$ is a restrictable equivalence.

In both cases, real $\mathrm{I}_{\mathbb{T}}^{X}$ is fully faithful, thus inducing an equivalence between $\mathrm{D}^{b}(\mathscr{A})$ and its essential image.

Proof (i) Consider the filtered bounded derived category $\operatorname{DF}^{b}(\mathscr{A})$ as an f-category over $\mathrm{D}^{b}(\mathscr{A})$ and let $(X, \theta)$ be an f-category over $\mathrm{D}^{b}(\mathscr{B})$ defined by $X=\operatorname{DF}^{b}(\mathscr{A})$ and $\theta=\theta^{\prime} \phi^{-1}$, where $\theta^{\prime}$ is the natural inclusion of $\mathrm{D}^{b}(\mathscr{A})$ in $\operatorname{DF}^{b}(\mathscr{A})$ (see Example 3.6). This choice of f-categories over $\mathrm{D}^{b}(\mathscr{A})$ and $\mathrm{D}^{b}(\mathscr{B})$ guarantees, trivially, that 
the identity functor on $\operatorname{DF}^{b}(\mathscr{A})$ is an f-lifting of $\phi$. Now, the functor $\phi$ is t-exact with respect to the standard t-structure in $\mathrm{D}^{b}(\mathscr{A})$ and the t-structure $\mathbb{T}$ in $\mathrm{D}^{b}(\mathscr{B})(\mathbb{T}$ was chosen for this purpose) and, thus, $\mathcal{H}(\mathbb{T})=\phi(\mathscr{A})$. Then Theorem 3.13 shows that real $\left.\right|_{\mathbb{T}} ^{x} \circ \mathrm{D}^{b}\left(\phi^{0}\right) \cong \phi \circ$ real $\left.\right|_{\mathbb{D}} ^{\mathrm{DF}(\mathscr{A})}$, where real $\mathrm{l}_{\mathbb{D}}^{\mathrm{DF}(\mathscr{A})}$ denotes the realisation of the standard t-structure in $\mathrm{D}^{b}(\mathscr{A})$ with respect to the filtered derived category of $\mathscr{A}$. Since by Proposition 3.14, real $\left.\right|_{\mathbb{D}} ^{\mathrm{DF}(\mathscr{A})}$ is naturally equivalent to $\operatorname{Id}_{\mathrm{D}^{b}(\mathscr{A})}$, we get that $\phi \cong \operatorname{real}_{\mathbb{T}}^{\mathcal{X}} \circ \mathrm{D}^{b}\left(\phi^{0}\right)$.

(ii) Consider the f-category $(X, \theta):=\left(\operatorname{DF}(\mathscr{A}), \theta^{\prime} \Phi^{-1}\right)$ over $\mathrm{D}(\mathscr{B})$, where now $\theta^{\prime}$ is the unbounded version of the functor stated in part (i). Then the first statement can be proved analogously to (i). The second statement, follows from Example 3.16(i).

Finally, note that in the above cases, since both $\phi$ and $\mathrm{D}^{b}\left(\phi^{0}\right)$ (respectively, $\Phi$ and $\mathrm{D}^{b}\left(\Phi^{0}\right)$ ) are fully faithful, then so is real $\mathbb{T}_{\mathbb{T}}^{X}$, finishing the proof.

The above statement is not particularly surprising. Given a derived category, any equivalence with another derived category yields an obvious new f-enhancement. The proposition translates this in terms of functors. The motto could be studying derived equivalence functors corresponds to studying f-enhancements. If, however, we want to study realisation functors with respect to fixed f-categories (for example, filtered derived categories), the problem resides then on the f-lifting property, as we will see in Sect. 5.

\section{Silting and cosilting $t$-structures}

We will now discuss a class of t-structures arising from certain objects (called silting or cosilting) in a triangulated category. Within this class, it will be possible to characterise exactly which associated realisation functors yield derived equivalences (see Sect. 5). These t-structures have appeared in the literature in various incarnations $[1,8,50,65,78]$. In this section we provide a general definition which covers, up to our knowledge, all the examples of silting complexes appearing in the literature, including non-compact ones. Furthermore, we introduce the dual notion of cosilting. Later, we specify to tilting and cotilting objects, observing how they can provide derived equivalences even when they are not compact.

In this section, $\mathcal{T}$ will denote a triangulated category. Given an object $X$ in $\mathcal{T}$, we will denote by $\operatorname{Add}(X)$ (respectively, $\operatorname{Prod}(X)$ ) the full subcategory of $\mathcal{T}$ consiting of all objects which are summands of a direct sum (respectively, of a direct product) of $X$. Note that without further assumptions, the category $\mathcal{T}$ might not admit arbitrary (set-indexed) coproducts or products of an object $X$. We will say that a triangulated category is TR5 if it has set-indexed coproducts and TR5* if it has set-indexed products. Recall from [62, Proposition 1.2.1] that, in a TR5 (respectively, TR5*) triangulated category, coproducts (respectively, products) of triangles are again triangles. Given an object $X$ in $\mathcal{T}$ and an interval $I$ of integers, we consider the following orthogonal subcategories of $\mathcal{T}$

$$
\begin{aligned}
& X^{\perp_{I}}=\left\{Y \in \mathcal{T} \mid \operatorname{Hom}_{\mathcal{T}}(X, Y[i])=0, \quad \forall i \in I\right\}, \\
& { }^{\perp_{I}} X=\left\{Y \in \mathcal{T} \mid \operatorname{Hom}_{\mathcal{T}}(Y, X[i])=0, \quad \forall i \in I\right\} .
\end{aligned}
$$

If the interval $I$ is unbounded, we often replace it by symbols such as $>n,<n, \geq n, \leq n$, $\neq n$ (with $n \in \mathbb{Z}$ ) with the obvious associated meaning. We say that an object $X$ generates $\mathcal{T}$ if $X^{\perp_{\mathbb{Z}}}=0$ and it cogenerates $\mathcal{T}$ if $\perp_{\mathbb{Z}} X=0$. Recall also that an object $X$ in a TR5 triangulated category is said to be compact if $\operatorname{Hom}_{\mathcal{T}}(X,-)$ commutes with coproducts. Here is an informal overview of this section. 


\section{Subsection 4.1: (Co)Silting objects in triangulated categories}

- We introduce the notion of silting (respectively, cosilting) objects in a triangulated category and list some examples and properties. In particular, we see in Proposition 4.3 that the hearts of the associated t-structures have a projective generator (respectively, an injective cogenerator).

\section{Subsection 4.2: Bounded (co)silting objects}

- We show that silting objects in derived categories of Grothendieck categories admit a more familiar description (Proposition 4.13).

- We define bounded (co)silting objects through the requirement that their associated tstructures restrict to bounded derived categories. This is necessary for the applications in Sects. 5 and 6.

- We prove that bounded silting objects in $\mathrm{D}(R)$, for a ring $R$, lie in $\mathrm{K}^{b}$ (Proj- $R$ ) (Proposition 4.17).

\section{1 (Co)Silting objects in triangulated categories}

We begin with the key notions for this section.

Definition 4.1 An object $M$ in a triangulated category $\mathcal{T}$ is called:

- silting if $\left(M^{\perp>0}, M^{\perp<0}\right)$ is a t-structure in $\mathcal{T}$ and $M \in M^{\perp_{>0}}$;

- cosilting if $\left({ }^{\perp_{<0}} M,{ }^{\perp>0} M\right)$ is a t-structure in $\mathcal{T}$ and $M \in{ }^{\perp>0} M$;

- tilting if it is silting and $\operatorname{Add}(M) \subset M^{\perp \neq 0}$;

- cotilting if it is cosilting and $\operatorname{Prod}(M) \subset{ }^{\perp \neq 0} M$.

We say that a t-structure is silting (respectively, cosilting, tilting or cotilting) if it arises as above from a silting (respectively, cosilting, tilting or cotilting) object.

Note that, in parallel work [65], silting objects are defined in an equivalent way.

It is clear from the definition that an object $M$ is silting in $\mathcal{T}$ if and only if $M$ is cosilting in the opposite category $\mathcal{T}^{o p}$. Hence, as we will see, many facts about silting can easily be dually stated for cosilting. A first easy observation, for example, is that if $M$ is silting, then $\operatorname{Add}(M)$ lies in $M^{\perp>0}$ and, dually, if $M$ is cosilting, then $\operatorname{Prod}(M)$ lies in ${ }^{\perp>0} M$.

Recall that an object $X$ in an abelian category $\mathscr{A}$ is a generator (respectively, a cogenerator) if $\operatorname{Hom}_{\mathscr{A}}(X,-)$ (respectively, $\left.\operatorname{Hom}_{\mathscr{A}}(-, X)\right)$ is a faithful functor. It is well-known (see, for example, [76, Propositions IV.6.3 and IV.6.5]) that a projective (respectively, injective) object $X$ is a generator (respectively, cogenerator) if and only if $\operatorname{Hom}_{\mathscr{A}}(X, Y) \neq 0$ (respectively, $\operatorname{Hom}_{\mathscr{A}}(Y, X) \neq 0$ ) for all $Y$ in $\mathscr{A}$. If $\mathscr{A}$ is cocomplete, then $G$ is a generator if and only if every object in $\mathscr{A}$ is isomorphic to a quotient of a coproduct of copies of $G$ [76, Proposition IV.6.2].

Example 4.2 The following span some expected classes of examples.

(i) Let $\mathcal{T}$ be a TR5 triangulated category. Then it follows from [1, Corollary 4.7] that any silting object in the sense of Aihara and Iyama in [1] is silting according to our definition. In fact, our definition of silting is motivated by that result. In particular, any tilting object in a TR5 triangulated category, as defined in [17] is tilting according to our definition. 
(ii) Let $\mathscr{A}$ be an abelian category. If $\mathscr{A}$ has a projective generator $P$, then $P$ is a silting (in fact, tilting) object in $\mathrm{D}(\mathscr{A})$ and the associated silting t-structure is the standard one. If $\mathscr{A}$ has an injective cogenerator $E$, then $E$ is a cosilting (in fact, cotilting) object in $\mathrm{D}(\mathscr{A})$ and the associated cosilting t-structure is also the standard one. For a proof, see Lemma 4.10 and Remark 4.11.

(iii) Let $\mathscr{A}$ be an abelian category with a projective generator $P$ (respectively, an injective cogenerator $E$ ) and $\mathcal{T}$ a triangulated category. If $\Phi: \mathrm{D}(\mathscr{A}) \longrightarrow \mathcal{T}$ is a triangle equivalence, then $\Phi(P)$ is a tilting object in $\mathcal{T}$ (respectively, $\Phi(E)$ is a cotilting object in $\mathcal{T})$.

(iv) Let $\mathscr{A}$ be a Grothendieck category. Then any 1-tilting object $T$ in $\mathscr{A}$ in the sense of [28] is a tilting object in $D(\mathscr{A})$ according to our definition. It is easy to check that the t-structure associated to $T$ is the HRS-tilt ([38, Proposition 2.1]) corresponding to the torsion pair (Gen( $T$ ), $T^{\perp_{0}}$ ) in $\mathscr{A}$ (see [8, Theorem 4.9] for an analogous argument when $\mathscr{A}=$ Mod- $R$, for a ring $R$ ).

(v) Let $A$ be a ring. It follows from [8, Proposition 4.2] that any silting complex $M$ in $\mathrm{D}(A)$ following the definition in $[8,78]$ is silting according to our definition. This includes, in particular, any compact tilting complex, as originally defined by Rickard in [71].

(vi) Let $A$ be a ring. Then it is shown in [77, Theorem 4.5] that any (large) $n$-cotilting module is cotilting according to our definition. Moreover, it is also shown in [13] that any (large) $n$-tilting module is tilting according to our definition. For commutative rings, t-structures for tilting and cotilting modules were considered in [9].

(vii) Let $\Lambda$ be a finite dimensional algebra over a field $\mathbb{K}$. Given a compact object $M$ in $D(\Lambda)$, there is a natural equivalence $\operatorname{Hom}_{\mathbb{K}}\left(\operatorname{Hom}_{\mathrm{D}(\Lambda)}(M,-), \mathbb{K}\right) \cong \operatorname{Hom}_{\mathrm{D}(\Lambda)}(-, v M)$ of contravariant functors between $\mathrm{D}(A)$ and the category of abelian groups, where $v:=$ $-\otimes_{\Lambda}^{\mathbb{L}} \operatorname{Hom}_{\mathbb{K}}(\Lambda, \mathbb{K})$ is the Nakayama functor (see [54] for more details). Using this equivalence, it is easy to show that if $M$ is a compact silting object in $\mathrm{D}(\Lambda)$, then $v M$ is a cosilting object in $\mathrm{D}(\Lambda)$. Compact silting objects over finite dimensional algebras were the first ones to be studied, already in [50] and later in [48,51], among others.

Given a silting or a cosilting object $M$ in $\mathcal{T}$, we denote by $\mathbb{T}_{M}$ the associated silting or cosilting t-structure and by $\mathcal{H}_{M}$ its heart. Note that if $M$ is silting, then $\mathcal{H}_{M}=M^{\perp \neq 0}$ and if $M$ is cosilting, then $\mathcal{H}_{M}={ }^{\perp \neq 0} M$. Given such a t-structure, we denote by $\mathrm{H}_{M}^{n}: \mathcal{T} \longrightarrow \mathcal{H}_{M}$ the associated cohomology functors and by $\tau_{M}^{\leq n}$ and $\tau_{\bar{M}}^{\geq n}$ the corresponding truncation functors, for all $n \in \mathbb{Z}$.

Proposition 4.3 Let $\mathcal{T}$ be a triangulated category. If $M$ is a silting (respectively, cosilting) object in $\mathcal{T}$, then $M$ is a generator (respectively, cogenerator) of $\mathcal{T}, \mathbb{T}_{M}$ is a nondegenerate $t$-structure in $\mathcal{T}$ and $H_{M}^{0}(M)$ is a projective generator (respectively, injective cogenerator) in $\mathcal{H}_{M}$.

Proof Assume first that $M$ is silting. Given $Y$ in $\mathcal{T}$, consider the canonical triangle

$$
\tau_{M}^{\leq-1} Y \longrightarrow Y \longrightarrow \tau_{M}^{\geq 0} Y \longrightarrow\left(\tau_{M}^{\leq-1} Y\right)[1] \text {. }
$$

If $Y$ lies in $M^{\perp_{\mathbb{Z}}}$, it follows by applying $\operatorname{Hom}_{\mathcal{T}}(M,-)$ to the triangle and its rotations that $Y=0$ and, hence, $M$ is a generator in $\mathcal{T}$. From this fact, it easily follows that the t-structure $\mathbb{T}_{M}$ is nondegenerate. In fact, if an object $X$ lies in $M^{\perp>0}[k]$ for all $k \in \mathbb{Z}$, then $\operatorname{Hom}_{\mathcal{T}}(M, X[k+1])=0$ for all $k \in \mathbb{Z}$ and, thus, $X=0$; similarly, if an object $X$ lies in $M^{\perp<0}[k]$ for all $k \in \mathbb{Z}$, we get that $X=0$, as wanted. 
Now we show that $\mathrm{H}_{M}^{0}(M)$ is projective in $\mathcal{H}_{M}$, i.e. that $\operatorname{Ext}_{\mathcal{H}_{M}}^{1}\left(\mathrm{H}_{M}^{0}(M), X\right)=0$, for any $X$ in $\mathcal{H}_{M}$. From Theorem 3.11(ii), we have that $\operatorname{Ext}_{\mathcal{H}_{M}}^{1}\left(\mathrm{H}_{M}^{0}(M), X\right) \cong \operatorname{Hom}_{\mathcal{T}}\left(\mathrm{H}_{M}^{0}(M), X[1]\right)$. Since $X$ lies in $M^{\perp_{>} 0}$, we have that $\operatorname{Hom}_{\mathcal{T}}(M, X[1])=0$ and, since $X[1]$ lies in $M^{\perp_{<0}}$ [1] and $\left(\tau_{M}^{\leq-1} M\right)[1]$ in $M^{\perp>0}[2]$, we conclude that $\operatorname{Hom}_{\mathcal{T}}\left(\left(\tau_{M}^{\leq-1} M\right)[1], X[1]\right)=0$. Using the above triangle for $Y=M$, where $\tau_{M}^{\geq 0}(M)=\mathrm{H}_{M}^{0}(M)$, it follows that $\operatorname{Hom}_{\mathcal{T}}\left(\mathrm{H}_{M}^{0}(M), X[1]\right)=0$, as wanted.

To show that a projective object in $\mathcal{H}_{M}$ is a generator, it is enough to show that it has non-zero morphisms to any object in $\mathcal{H}_{M}$. Since $\mathcal{H}_{M}=M^{\perp \neq 0}$ and $M$ generates $\mathcal{T}$, we have $\operatorname{Hom}_{\mathcal{T}}(M, X) \neq 0$ for any non-zero $X$ in $\mathcal{H}_{M}$. Therefore, using again the above triangle for $Y=M$, we can conclude that $\operatorname{Hom}_{\mathcal{T}}\left(\mathrm{H}_{M}^{0}(M), X\right) \cong \operatorname{Hom}_{\mathcal{T}}(M, X) \neq 0$, showing that $\mathrm{H}_{M}^{0}(M)$ is a projective generator.

If $M$ is cosilting, for any $Y$ in $\mathcal{T}$ we may again consider the above triangle and apply the dual arguments to those in the previous paragraphs to see that $M$ is a cogenerator in $\mathcal{T}, \mathbb{T}_{M}$ is nondegenerate and $\mathrm{H}_{M}^{0}(M)$ is an injective cogenerator of $\mathcal{H}_{M}$. Note that since $M$ is cosilting, for $Y=M$ we have $\tau_{M}^{\leq 0}(M)=\mathrm{H}_{M}^{0}(M)$.

Remark 4.4 From [16, Proposition 1.3.7] (see also [36, Theorem IV.4.11]), since silting tstructures are nondegenerate, they can be cohomologically described, i.e. for a silting object $M$ in $\mathcal{T}$, we have

$$
M^{\perp>0}=\left\{X \in \mathcal{T} \mid H_{M}^{k}(X)=0, \forall k>0\right\} \quad M^{\perp<0}=\left\{X \in \mathcal{T} \mid H_{M}^{k}(X)=0, \quad \forall k<0\right\} .
$$

Dually, cosilting t-structures are nondegenerate and can also be, therefore, cohomologically described.

With further assumptions on $\mathcal{T}$ we can say more about hearts of silting and cosilting tstructures. We say that a t-structure in a TR5 (respectively, TR5*) triangulated category is smashing (respectively, cosmashing) if its coaisle (respectively, its aisle) is closed under coproducts (respectively, products).

Lemma 4.5 Let $\mathcal{T}$ be a triangulated category and $M$ an object in $\mathcal{T}$.

(i) [67, Propositions 3.2 and 3.3] If $\mathcal{T}$ is TR5 (respectively, TR5*), then the heart of any $t$-structure in $\mathcal{T}$ has set-indexed coproducts (respectively, products). Furthermore, if the t-structure is smashing (respectively, cosmashing), then coproducts (respectively, products) are exact in the heart.

(ii) If $\mathcal{T}$ is TR5 and $M$ is a silting object of $\mathcal{T}$, then the following statements hold.

(a) $\operatorname{Add}(M)=M^{\perp_{>0}} \cap^{\perp_{0}}\left(M^{\perp_{>0}}[1]\right)$;

(b) If $\mathcal{T}$ is also TR5*, $\left(M^{\perp>0}, M^{\perp<0}\right)$ is a cosmashing $t$-structure and products are exact in $\mathcal{H}_{M}$.

(iii) If $\mathcal{T}$ is TR5* and $M$ is a cosilting object of $\mathcal{T}$, then the following statements hold.

(a) $\operatorname{Prod}(M)={ }^{\perp_{>0}} M \cap\left({ }_{>0} M[-1]\right)^{\perp_{0}}$;

(b) If $\mathcal{T}$ is also TR5, $\left({ }^{\perp_{<0}} M,{ }^{\perp>0} M\right)$ is a smashing $t$-structure and coproducts are exact in $\mathcal{H}_{M}$.

Proof Statement (i) was proved in [67, Propositions 3.2 and 3.3]. Recall that in a TR5 (respectively, TR5*) triangulated category, given a family of objects $\left(X_{i}\right)_{i \in I}$, for some set $I$, in the heart $\mathcal{H}$ of a t-structure $(\mathbb{T} \leq 0, \mathbb{T} \geq 0)$, their coproduct (respectively, their product) in $\mathcal{H}$ is given by $\tau^{\geq 0} \bigsqcup_{i \in I} X_{i}$ (respectively, $\tau=0 \prod_{i \in I} X_{i}$ ), where the coproduct $\bigsqcup_{i \in I} X_{i}$ (respectively, the product $\prod_{i \in I} X_{i}$ ) is taken in $\mathcal{T}$. 
We now prove (ii) (the arguments for (iii) are dual). The proof of (ii)(a) essentially mimics that of [8, Lemma 4.5], as follows. Let $M$ be a silting object in $\mathcal{T}$. It is clear that $\operatorname{Add}(M) \subseteq M^{\perp_{>0}} \cap{ }^{\perp_{0}}\left(M^{\perp_{>0}}[1]\right)$. Conversely, let $X$ lie in $M^{\perp_{>0}} \cap^{\perp_{0}}\left(M^{\perp_{>0}}[1]\right)$ and let $I$ be the set $\operatorname{Hom}_{\mathcal{T}}(M, X)$. Consider the triangle

$$
K \longrightarrow M^{(I)} \stackrel{u}{\longrightarrow} X \stackrel{v}{\longrightarrow} K[1],
$$

where $u$ is the universal map from $M$ to $X$. We show that $v=0$ and, thus, $u$ splits. Since $\operatorname{Hom}_{\mathcal{T}}(M, u)$ is surjective and $\operatorname{Hom}_{\mathcal{T}}\left(M, M^{(I)}[1]\right)=0$, it follows that $\operatorname{Hom}_{\mathcal{T}}(M, K[1])=0$. Similarly, we have that, for all $i \geq 1, \operatorname{Hom}_{\mathcal{T}}(M, K[i])=0$. Thus, $K$ lies in $M^{\perp>0}$. Since $X$ lies in ${ }^{\perp_{0}}\left(M^{\perp_{>0}}[1]\right)$, it follows that $v=0$. Finally, to prove (ii)(b), it suffices to note that $M^{\perp>0}$ is closed under products whenever they exist.

Definition 4.6 Let $\mathcal{T}$ be a triangulated category and $M$ and $N$ silting (respectively, cosilting) objects of $\mathcal{T}$. We say that $M$ and $N$ are equivalent if they yield the same silting (respectively, cosilting) t-structure.

By Lemma 4.5, two silting (respectively, cosilting) objects $M$ and $N$ in a TR5 (respectively, TR5*) triangulated category are equivalent if and only if $\operatorname{Add}(M)=\operatorname{Add}(N)$ (respectively, $\operatorname{Prod}(M)=\operatorname{Prod}(N))$.

The following corollary brings us to the more familiar setting of compact tilting objects, where the endomorphism ring of a fixed tilting object plays an important role (see also [17, Corollary 4.2] and [40, Theorem 1.3]).

Corollary 4.7 Let $\mathcal{T}$ be a TR5 triangulated category and $M$ a silting object which is equivalent to a compact one. Then the heart $\mathcal{H}_{M}$ is equivalent to $\operatorname{Mod}_{-E^{-}} \operatorname{Cd}_{\mathcal{T}}\left(H_{M}^{0}(M)\right)$.

Proof From Proposition 4.3, $\mathrm{H}_{M}^{0}(M)$ is a projective generator of $\mathcal{H}_{M}$. Since equivalent silting objects yield the same t-structure, assume without loss of generality that $M$ is compact. Using the triangle

$$
\tau_{M}^{\leq-1} M \longrightarrow M \longrightarrow \mathrm{H}_{M}^{0}(M) \longrightarrow\left(\tau_{M}^{\leq-1} M\right)[1]
$$

it is easy to see that there is an isomorphism of functors $\operatorname{Hom}_{\mathcal{H}_{M}}\left(\mathrm{H}_{M}^{0}(M),-\right) \cong$ $\left.\operatorname{Hom}_{\mathcal{T}}(M,-)\right)_{\mid \mathcal{H}_{M}}$. Since $M$ is compact, the coaisle $M^{\perp<0}$ is closed under coproducts. By the proof of Lemma 4.5(i), coproducts in $\mathcal{H}_{M}$ coincide with coproducts in $\mathcal{T}$ and, thus, both functors above commute with coproducts. This shows that $\mathrm{H}_{M}^{0}(M)$ is small in $\mathcal{H}_{M}$. The result then follows by classical Morita theory.

One may ask for another way of describing the (co)aisle of a (co)silting t-structure in terms of the given (co)silting object $M$. We will see that there is a smallest (co)aisle containing $M$ and that it coincides with the (co)aisle of the (co)silting t-structure. For that, recall that a subcategory of a triangulated category is said to be suspended (respectively, cosuspended) if it is closed under extensions and positive (respectively, negative) iterations of the suspension functor. Regarding these subcategories, one has the following useful lemma coming from [2].

Lemma 4.8 [2, Lemma 3.1] Let $\mathcal{T}$ be a triangulated category. Let $X$ be an object in $\mathcal{T}$ and $\mathcal{S}$ be the smallest suspended (respectively, cosuspended) subcategory containing $X$ and closed under summands and all existing coproducts (respectively, products). Then $\mathcal{S}^{\perp_{0}}=X^{\perp} \leq$ (respectively, ${ }^{\perp_{0}} \mathcal{S}={ }^{\perp} \leq 0 \mathrm{X}$ ). 
Proof The proof in [2, Lemma 3.1] does not depend on the existence of arbitrary coproducts. Also, the arguments can easily be dualised to obtain the cosuspended case.

The smallest aisle of a triangulated category $\mathcal{T}$ containing an object $X$, denoted by aisle $(X)$, is known to exist whenever $\mathcal{T}$ is the derived category of a Grothendieck abelian category or whenever $X$ is a compact object ([2, Theorems 3.4 and A.1]). We show that it also exists for a silting object in any triangulated category (the dual statement about the smallest coaisle containing a cosilting object also holds).

Proposition 4.9 Let $M$ be a silting (respectively, cosilting) object in a triangulated category $\mathcal{T}$. Then the smallest aisle (respectively, coaisle) containing $M$ exists and it coincides with $M^{\perp>0}$ (respectively, ${ }^{\perp>0} M$ ).

Proof Let us prove the silting case (the cosilting case can be proved dually). Let $M$ be a silting object and let $\mathcal{S}$ denote the smallest suspended subcategory of $\mathcal{T}$ containing $M$ and closed under summands and existing coproducts. Let $\mathcal{V}$ be an aisle containing $M$ (which exists since $M^{\perp>0}$ is an aisle where $M$ lies). Then certainly we have that $\mathcal{V} \supseteq \mathcal{S}$ since aisles are suspended, closed under summands and existing sums. Hence, we also have ${ }^{\perp_{0}}\left(\mathcal{V}^{\perp_{0}}\right) \supseteq{ }^{\perp_{0}}\left(\mathcal{S}^{\perp_{0}}\right)$. But in this inclusion, the left-hand side coincides with $\mathcal{V}$ since $\mathcal{V}$ is an aisle and the right-hand side clearly contains ${ }^{\perp_{0}}\left(M^{\perp_{\leq 0}}\right)$ (it in fact coincides with it by Lemma 4.8). Since $M$ is silting, $M^{\perp_{>0}}={ }^{\perp_{0}}\left(M^{\perp_{\leq 0}}\right)$ and, thus, $\mathcal{V}$ contains $M^{\perp_{>0}}$, showing that $M^{\perp_{>0}}$ is indeed the smallest aisle containing $M$.

\subsection{Bounded (co)silting objects}

We will now discuss examples of silting and cosilting objects in derived categories of abelian categories. As suggested by Example 4.2, a good assumption on the underlying abelian category $\mathscr{A}$ is the presence of a generator or a cogenerator. Particularly well-behaved examples of abelian categories with a generator (and a cogenerator) are Grothendieck abelian categories. An abelian category $\mathscr{A}$ is said to be Grothendieck if it is a cocomplete abelian category with exact direct limits and a generator. It is well-known that if $\mathscr{A}$ is Grothendieck, $\mathrm{D}(\mathscr{A})$ is both TR5 and TR5* (see also Remark 6.7). Moreover, $\mathscr{A}$ has an injective cogenerator and it also follows from [3, Theorem 5.4] that every object in $\mathrm{D}(\mathscr{A})$ admits a homotopically injective coresolution.

We begin by collecting some useful observations that clarify the relation between the existence of a generator (or cogenerator) in an abelian category $\mathscr{A}$ and the standard t-structure in $\mathrm{D}(\mathscr{A})$. The assumptions in the lemma include the particular case when $\mathscr{A}$ is Grothendieck.

Lemma 4.10 Let $\mathscr{A}$ be an abelian category with a generator $G$. Suppose that every object in $\mathrm{D}(\mathscr{A})$ admits a homotopically injective coresolution or that $G$ is a projective object in $\mathscr{A}$. Then we have:

(i) For any object $X$ in $\mathrm{D}(\mathscr{A})$ and any $i \in \mathbb{Z}$, if $\operatorname{Hom}_{\mathrm{D}(\mathscr{A})}(G, X[i])=0$ then $\mathrm{H}_{0}^{i}(X)=0$;

(ii) If $\mathrm{D}(\mathscr{A})$ is TR5, the smallest aisle of $\mathrm{D}(\mathscr{A})$ containing $G$ exists and coincides with the standard aisle $\mathbb{D}^{\leq 0}$;

(iii) Consider the following statements:

(a) $G$ is projective in $\mathscr{A}$;

(b) For any object $X$ in $\mathrm{D}(\mathscr{A})$ and any $i \in \mathbb{Z}, \mathrm{H}_{0}^{i}(X)=0$ if and only if $\operatorname{Hom}_{\mathrm{D}(\mathscr{A})}(G, X[i])=0$;

(c) $G$ is a silting object in $\mathrm{D}(\mathscr{A})$; 
(d) $G$ is a tilting object in $\mathrm{D}(\mathscr{A})$.

Then $(\mathrm{a}) \Longrightarrow(\mathrm{b}) \Longrightarrow(\mathrm{c}) \Longleftrightarrow(\mathrm{d})$. If, furthermore, $\mathrm{D}(\mathscr{A})$ is TR5, then the statements are equivalent.

Proof (i) Assume that $\mathrm{H}_{0}^{i}(X) \neq 0$. We will construct a non-trivial map from $G$ to $X[i]$. By our hypothesis on $\mathrm{D}(\mathscr{A})$, without loss of generality we may assume that $X=\left(X^{j}, d^{j}\right)_{j \in \mathbb{Z}}$ is a complex of injective objects in $\mathscr{A}$ or that $G$ is projective in $\mathscr{A}$. Thus, we have $\operatorname{Hom}_{\mathrm{D}(\mathscr{A})}(G, X[i])=\operatorname{Hom}_{\mathrm{K}(\mathscr{A})}(G, X[i])$. Denote by $q: \operatorname{Ker} d^{i} \longrightarrow \mathrm{H}_{0}^{i}(X)$ the natural projection map. Since $G$ is a generator and $\mathrm{H}_{0}^{i}(X) \neq 0, \operatorname{Hom}_{\mathscr{A}}(G, q) \neq 0$ and, thus, there is a map $f: G \longrightarrow \operatorname{Ker} d^{i}$ such that $q \circ f \neq 0$, i.e. $f$ does not factor through the natural inclusion $k: \operatorname{Im} d^{i-1} \longrightarrow \operatorname{Ker} d^{i}$. Consider the following diagram

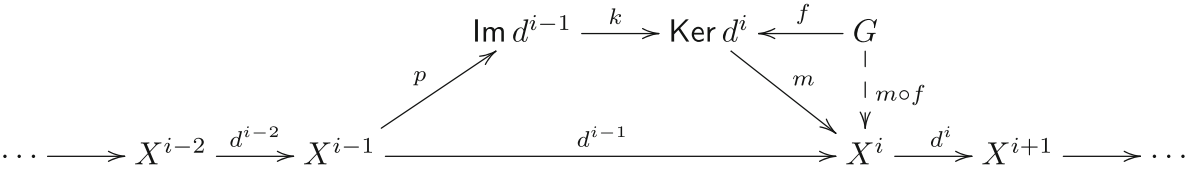
where $m$ and $p$ are the obviously induced maps. Then it is clear that the map $m \circ f$ lies in $\operatorname{Hom}_{\mathrm{K}(\mathscr{A})}(G, X[i])$ and it is non-zero by the choice of $f$.

(ii) Let $\mathcal{S}$ denote the smallest suspended subcategory of $\mathrm{D}(\mathscr{A})$ containing $G$ and closed under coproducts. If aisle $(G)$ exists, it contains $\mathcal{S}$ and, since $G$ lies in $\mathbb{D} \leq 0$, it is also contained in $\mathbb{D}^{\leq 0}$. Hence, if one shows that $\mathcal{S}=\mathbb{D}^{\leq 0}$ the statement follows. By Lemma 4.8, it follows that $\mathcal{S}^{\perp_{0}}=G^{\perp^{\leq 0}}$. It is clear that $\mathbb{D}^{\geq 1} \subseteq G^{\perp \leq 0}$. On the other hand, given $X$ in $G^{\perp \leq 0}$, it follows from (i) that $\mathrm{H}_{0}^{i}(X)=0$ for all $i \leq 0$. Hence, we have that $\mathbb{D}^{\geq 1} \supseteq G^{\perp_{\leq 0}}$ and ${ }^{\perp_{0}}\left(\mathcal{S}^{\perp_{0}}\right)=\mathbb{D}^{\leq 0}$ as wanted.

(iii) (a) $\Longrightarrow$ (b): Assume that $G$ is projective and suppose that $\mathrm{H}_{0}^{i}(X)=0$ (which, following the notation of the diagram above, is equivalent to $k: \operatorname{Im} d^{i-1} \longrightarrow \operatorname{Ker} d^{i}$ being an isomorphism). Let $g: G \longrightarrow X[i]$ be a map in $\mathrm{D}(\mathscr{A})$. Since $G$ is projective, this is also a map in $K(\mathscr{A})$ and, therefore, $d^{i} g=0$, i.e. $g$ induces a map $\tilde{g}: G \longrightarrow \operatorname{Ker} d^{i}$. Since $k: \operatorname{Im} d^{i-1} \longrightarrow \operatorname{Ker} d^{i}$ is an isomorphism by assumption, $\tilde{g}$ factors through $k$. This factorisation yields a homotopy, thus showing that $g=0$, as wanted.

(b) $\Longrightarrow\left(\right.$ c): From (b) it is clear that we have the equality $\left(G^{\perp>0}, G^{\perp<0}\right)=\left(\mathbb{D}^{\leq 0}, \mathbb{D}^{\geq 0}\right)$, thus showing that $\left(G^{\perp>0}, G^{\perp<0}\right)$ is a t-structure. Since $G$ lies in $\mathbb{D}^{\leq 0}$, then it also lies in $G^{\perp>0}$, showing that it is silting.

(c) $\Longleftrightarrow(\mathrm{d})$ : Objects of $\mathscr{A}$ do not admit negative self-extensions. In particular, $G$ lies in $G^{\perp<0}$. Therefore, $G$ is silting if and only if it is tilting.

When $\mathrm{D}(\mathscr{A})$ is TR5, we can also prove $(\mathrm{d}) \Longrightarrow(\mathrm{a})$ : If $G$ is a tilting object in $\mathrm{D}(\mathscr{A})$, then $\left(G^{\perp>0}, G^{\perp_{<0}}\right)$ is a t-structure and $G$ lies in $\mathcal{H}_{G}$, where it is projective (Proposition 4.3). From Proposition 4.9 and item (ii) above, we have that $G^{\perp>0}=\operatorname{aisle}(G)=\mathbb{D}^{\leq 0}$. Hence, the associated tilting t-structure is the standard one and $\mathcal{H}_{G}=\mathscr{A}$, showing that $G$ is projective in $\mathscr{A}$.

Remark 4.11 Note that if $\mathscr{A}$ has an injective cogenerator $E$, then one can argue as in (iii) above to show that $E$ is a cotilting object in $\mathrm{D}(\mathscr{A})$ and that for any object $X$ in $\mathrm{D}(\mathscr{A})$ and any $i \in \mathbb{Z}, \mathrm{H}_{0}^{i}(X)=0$ if and only if $\operatorname{Hom}_{\mathrm{D}(\mathscr{A})}(X, E[-i])=0$, i.e. the associated cotilting $\mathrm{t}$-structure is the standard one.

The above lemma and the intrinsic properties of tilting t-structures yield the following corollary. 
Corollary 4.12 Let $\mathscr{A}$ be an abelian category such that $\mathrm{D}(\mathscr{A})$ is TR5 and TR5*. If $\mathscr{A}$ has a projective generator (respectively, an injective cogenerator), then $\mathscr{A}$ has exact products (respectively, coproducts). In particular, if $\mathscr{A}$ is a Grothendieck abelian category with a projective generator, then $\mathscr{A}$ has exact products.

Proof From Lemma 4.10, the projective generator $G$ is a tilting object in $\mathrm{D}(\mathscr{A})$ whose associated tilting t-structure is the standard one. From Lemma 4.5(ii)(b) it then follows that $\mathcal{H}_{G} \cong \mathscr{A}$ has exact products. The dual statement follows from Remark 4.11 and Lemma 4.5(iii)(b).

As mentioned earlier, if $\mathscr{A}$ is a Grothendieck category, the smallest aisle of $D(\mathscr{A})$ containing an object always exists ([2, Theorem 3.4]). Using this fact, silting objects become easier to describe. This description covers the previous definitions of silting objects occurring in the literature $[1,8,48,50,51,78]$.

Proposition 4.13 Let $\mathscr{A}$ be a Grothendieck category and $M$ an object in $\mathrm{D}(\mathscr{A})$. Then $M$ is silting if and only if the following three conditions hold:

(i) $\operatorname{Hom}_{\mathrm{D}(\mathscr{A})}(M, M[k])=0$, for all $k>0$;

(ii) $M$ generates $\mathrm{D}(\mathscr{A})$;

(iii) $M^{\perp>0}$ is closed under coproducts.

Proof If $M$ is silting, it follows from Proposition 4.3 that $M$ is a generator and, thus, it satisfies the listed conditions. Conversely, suppose $M$ satisfies (i), (ii) and (iii). Since $\mathscr{A}$ is a Grothendieck category, it follows from [2, Theorem 3.4] that aisle $(M)$ coincides with the smallest subcategory containing $M$ and closed under positive shifts, extensions and coproducts. As a consequence, and by assumption on $M^{\perp>0}$, we have that aisle $(M)^{\perp_{0}}=M^{\perp_{\leq 0}}$ (by Lemma 4.8) and aisle $(M) \subseteq M^{\perp>0}$. We will show the reverse inclusion, thus proving that $M$ is silting. Let $X$ be an object in $M^{\perp>0}$ and consider a triangle

$$
Y \longrightarrow X \longrightarrow Z \longrightarrow Y[1]
$$

such that $Y$ lies in aisle $(M)$ and $Z$ lies in aisle $(M)^{\perp_{0}}=M^{\perp_{\leq 0}}$. We show that $Z=0$. Note that $\operatorname{Hom}_{\mathrm{D}(\mathscr{A})}(M, Z[k])=0$, for all $k \leq 0$. Let $k<0$ and consider a map $f: M[k] \longrightarrow Z$. Since aisle $(M) \subseteq M^{\perp>0}$ (and, thus, $\operatorname{Hom}_{\mathrm{D}(\mathscr{A})}(M[k], Y[1])=0$ ), it follows that there is $\bar{f}: M[k] \longrightarrow X$ which, by assumption on $X$, must vanish. Therefore, $f=0$ and since $M$ generates $\mathrm{D}(\mathscr{A})$, we get that $Z=0$. From the above triangle we infer that $X$ lies in aisle $(M)$.

This proposition shows, in particular, that the compact silting (and tilting) complexes in derived module categories that appear abundantly in the literature fit in our definition. We will now explore in more detail the connection between the (not necessarily compact) silting complexes defined in $[8,78]$ and silting objects as defined here. For this purpose we need the notion of a bounded (co)silting object, which will play an important role in the coming sections. This concept is defined via a property of the associated t-structure.

Lemma 4.14 Let $\mathscr{A}$ be an abelian category and let $\mathbb{T}=\left(\mathbb{T}^{\leq 0}, \mathbb{T}^{\geq 0}\right)$ be a $t$-structure in $\mathrm{D}(\mathscr{A})$ with heart $\mathcal{H}$. Then the following are equivalent.

(i) The full subcategory $\mathrm{D}(\mathscr{A})^{b_{\mathbb{T}}}$ of $\mathrm{D}(\mathscr{A})$ with objects $\mathrm{Ob} \mathrm{D}(\mathscr{A})^{b(\mathbb{T})}=\bigcup_{n, m \in \mathbb{Z}} \mathrm{Ob} \mathbb{T}^{\leq n} \cap$ $\mathbb{T}^{\geq m}$ coincides with $\mathrm{D}^{b}(\mathscr{A})$. 
(ii) $\mathbb{T} \cap \mathrm{D}^{b}(\mathscr{A}):=\left(\mathbb{T} \leq 0 \cap \mathrm{D}^{b}(\mathscr{A}), \mathbb{T}^{\geq 0} \cap \mathrm{D}^{b}(\mathscr{A})\right)$ is a bounded $t$-structure in $\mathrm{D}^{b}(\mathscr{A})$ and $\mathcal{H} \subseteq \mathrm{D}^{b}(\mathscr{A})$.

If $\mathscr{A}$ is Grothendieck, then the above conditions are furthermore equivalent to the following ones.

(iii) There are integers $n \leq m$ such that $\mathbb{D} \leq n \subseteq \mathbb{T} \leq 0 \subseteq \mathbb{D} \leq m$.

(iv) $\mathrm{Ob} \mathrm{D}^{-}(\mathscr{A})=\bigcup_{n \in \mathbb{Z}} \mathrm{Ob} \mathbb{T}^{\leq 0}[n]$ and $\mathrm{Ob} \mathrm{D}^{+}(\mathscr{A})=\bigcup_{n \in \mathbb{Z}} \mathrm{Ob} \mathbb{T}^{\geq 0}[n]$.

Proof (i) $\Longrightarrow$ (ii): We first observe that $\left(\mathbb{T}^{\leq 0} \cap \mathrm{D}(\mathscr{A})^{b(\mathbb{T})}, \mathbb{T}^{\geq 0} \cap \mathrm{D}(\mathscr{A})^{b(\mathbb{T})}\right)$ is always a t-structure in $\mathrm{D}(\mathscr{A})^{b(\mathbb{T})}$. In fact, given an object $X$ in $\mathrm{D}(\mathscr{A})^{b(\mathbb{T})}$ it is clear that its truncations with respect to $\mathbb{T}, \tau_{\mathbb{T}}^{\leq 0} X$ and $\tau_{\mathbb{T}}^{\geq 1} X$, lie, respectively, in $\cup_{n \in \mathbb{Z}} \mathbb{T}^{\leq n}$ and in $\cup_{n \in \mathbb{Z}} \mathbb{T}^{\geq n}$. Each of these unions forms a triangulated subcategory of $\mathrm{D}(\mathscr{A})$. Since $X$ lies in both, it follows that so do its truncations. Hence, $\left(\mathbb{T}^{\leq 0} \cap \mathrm{D}(\mathscr{A})^{b(\mathbb{T})}, \mathbb{T}^{\geq 0} \cap \mathrm{D}(\mathscr{A})^{b(\mathbb{T})}\right)$ is indeed a t-structure and (ii) then follows immediately from (i).

(ii) $\Longrightarrow$ (i): Note that every object in $\mathbb{T}^{\leq n} \cap \mathbb{T}^{\geq m}$ can be obtained by a finite sequence of extensions in $\mathrm{D}(\mathscr{A})$ of objects in $\mathcal{H}$ (such sequences are often represented by Postnikov towers - see also [20, Lemma 2.3]). Since $\mathcal{H}$ lies in $\mathrm{D}^{b}(\mathscr{A})$ then so does every object in $\mathbb{T}^{\leq n} \cap \mathbb{T}^{\geq m}$. Hence $\mathrm{D}(\mathscr{A})^{b(\mathbb{T})}$ is contained in $\mathrm{D}^{b}(\mathscr{A})$. The converse inclusion also holds since $\mathbb{T} \cap \mathrm{D}^{b}(\mathscr{A})$ is a bounded t-structure in $\mathrm{D}^{b}(\mathscr{A})$.

Suppose now that $\mathscr{A}$ is a Grothendieck category with a generator $G$ and an injective cogenerator $E$.

(ii) $\Longrightarrow$ (iii): By assumption, $G$ lies in $\mathbb{T}^{\leq k}$ for some integer $k$. By Lemma 4.10, we have that aisle $(G)=\mathbb{D}^{\leq 0}$ and, thus, $\mathbb{D}^{\leq 0} \subseteq \mathbb{T}^{\leq k}$. Since, by assumption, $E$ lies in $\mathbb{T}^{\geq t}$ for some integer $t$ and $\mathbb{T}^{\geq t}$ is closed under negative shifts, using Remark 4.11 we see that $\mathbb{D}^{\leq 0}={ }^{\perp_{<0}} E \supseteq{ }^{\perp_{<0}}\left(\mathbb{T}^{\geq t}\right)={ }^{\perp_{0}}\left(\mathbb{T}^{\geq t+1}\right)$, as wanted.

(iii) $\Longrightarrow$ (iv): This is obvious.

(iv) $\Longrightarrow$ (ii): In order to see that the t-structure $\mathbb{T}$ restricts to $\mathrm{D}^{b}(\mathscr{A})$ we only need to show (as in (i) $\Longrightarrow$ (ii)) that given an object $X$ in $\mathrm{D}^{b}(\mathscr{A})$, its truncations with respect to $\mathbb{T}$ also lie in $\mathrm{D}^{b}(\mathscr{A})$. Consider the truncation triangle with respect to $\mathbb{T}$ given as follows

$$
\tau^{\leq 0} X \longrightarrow X \longrightarrow \tau^{\geq 1} X \longrightarrow\left(\tau^{\leq 0} X\right)[1] .
$$

By assumption, $\mathbb{T}^{\leq n} \subseteq \mathrm{D}^{-}(\mathscr{A})$ and, thus, $\tau^{\leq 0} X$ lies in $\mathrm{D}^{-}(\mathscr{A})$. Since $\mathrm{D}^{-}(\mathscr{A})$ is a triangulated subcategory and $X$ lies in $\mathrm{D}^{b}(\mathscr{A})$, we have that also $\tau^{\geq 1} X$ lies in $\mathrm{D}^{-}(\mathscr{A})$. Again by assumption, we also have that $\tau^{\geq 1} X$ lies in $\mathbb{T}^{\geq 1} \subseteq \mathrm{D}^{+}(\mathscr{A})$ and, thus, in $\mathrm{D}^{-}(\mathscr{A}) \cap \mathrm{D}^{+}(\mathscr{A})=\mathrm{D}^{b}(\mathscr{A})$. Since $\mathrm{D}^{b}(\mathscr{A})$ is triangulated, also $\tau=0$ lies in $\mathrm{D}^{b}(\mathscr{A})$. Hence $\mathbb{T} \cap \mathrm{D}^{b}(\mathscr{A})$ is a t-structure in $\mathrm{D}^{b}(\mathscr{A})$. Finally, it is clear from (iv) that $\mathbb{T} \cap \mathrm{D}^{b}(\mathscr{A})$ is bounded and that $\mathcal{H}(\mathbb{T})=\mathbb{T}^{\leq 0} \cap \mathbb{T}^{\geq 0} \subset \mathrm{D}^{-}(\mathscr{A}) \cap \mathrm{D}^{+}(\mathscr{A})=\mathrm{D}^{b}(\mathscr{A})$, proving (ii).

Definition 4.15 Let $\mathscr{A}$ be an abelian category. A silting (respectively, cosilting) object in $\mathrm{D}(\mathscr{A})$ is said to be bounded if the associated t-structure satisfies the equivalent conditions (i) and (ii) of Lemma 4.14.

Remark 4.16 (i) If $\mathscr{A}$ is an abelian category, any bounded tilting and bounded cotilting objects in $\mathrm{D}(\mathscr{A})$ lie in $\mathrm{D}^{b}(\mathscr{A})$ since they lie in their own associated hearts.

(ii) If $\mathscr{A}$ is a Grothendieck category and $M$ is a bounded silting object in $\mathrm{D}(\mathscr{A})$, then $M$ lies in $\mathrm{D}^{b}(\mathscr{A})$. From (iii) of Lemma 4.14, it is clear that there are integers $n \leq m$ such that $M$ lies in $\mathbb{D}^{\leq m}$ and $\mathbb{D}^{\leq n} \subseteq M^{\perp>0}$. For $E$ and injective cogenerator of $\mathscr{A}$, since $E[-n]$ lies in $\mathbb{D} \leq n$, we have that $\operatorname{Hom}_{\mathrm{D}(A)}(M, E[-n+k])=0$ for all $k>0$. In particular, it follows from Remark 4.11 that $M$ lies in $\mathbb{D}^{\geq n+1}$, proving that $M$ is a cohomologically bounded object. 
(iii) If $\mathscr{A}$ is a Grothendieck category with a projective generator $G$, then the dual arguments to the above example show that bounded cosilting objects also lie in $D^{b}(\mathscr{A})$.

We will see that, in the derived category of modules over a ring $A$, bounded silting objects are not only cohomologically bounded but they must also lie in $\mathrm{K}^{b}$ (Proj- $A$ ). Hence, they coincide with the silting complexes of $[8,78]$. Analogously, bounded cosilting objects in $\mathrm{D}(A)$ must lie in $\mathrm{K}^{b}(\operatorname{Inj}-(A))$. This can be shown using dual arguments to those in [8, Lemma 4.5]—we leave that to the reader.

Proposition 4.17 Let $A$ be a ring. Then a silting (respectively, cosilting) object is bounded in $\mathrm{D}(A)$ if and only if it lies in $\mathrm{K}^{b}$ (Proj- $\left.A\right)$ (respectively, $K^{b}(\operatorname{Inj}-A)$ ).

Proof By Lemma 4.14, the aisle of the silting t-structure associated to a bounded silting object in $\mathrm{D}(A)$ lies between shifts of the standard aisle. It then follows from Lemma 4.5(ii)(a) and $\left[8\right.$, Lemma 4.5] that bounded silting objects lie in $\mathrm{K}^{b}$ (Proj- $\left.A\right)$. For the converse see [78, Lemma 4.1 and Proposition 4.2].

We finish this section with an example of an unbounded silting object in the context of quiver representations of infinite quivers. Let $\mathbb{K}$ be a field. Given a (possibly infinite) locally finite quiver $Q$ (i.e. every vertex has only finitely many adjacent arrows), consider the path category of $Q$ to be the category whose objects are the vertices of $Q$ and whose morphisms between two vertices $i$ and $j$ are elements of the $\mathbb{K}$-vector space spanned by the paths between $i$ and $j$. We denote it by $\mathbb{K} Q$. Consider the associated category of functors $\mathcal{M}(\mathbb{K} Q):=\left((\mathbb{K} Q)^{o p}\right.$, Mod- $\left.\mathbb{K}\right)$, which is called the category of right modules over $\mathbb{K} Q$. If $Q$ is finite, $\mathcal{M}(\mathbb{K} Q)$ is equivalent to usual category of right modules over the path algebra, Mod- $\mathbb{K} Q$. Still, even when $Q$ is infinite, $\mathcal{M}(\mathbb{K} Q)$ is well-known to be a Grothendieck category (see, for example, [31]). Given a vertex $x$ of $Q$, we consider the projective object $P_{x}=\operatorname{Hom}_{\mathbb{K} Q}(x,-)$ in $\mathcal{M}(\mathbb{K} Q)$.

Example 4.18 Let $Q$ be the linearly oriented quiver of type $A_{\infty}$, i.e. the quiver

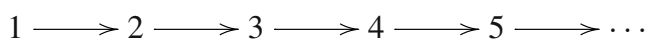

Consider the derived category $\mathrm{D}(\mathcal{M}(\mathbb{K} Q))$. We show, using Proposition 4.13, that

$$
M:=\bigoplus_{i \in \mathbb{N}} P_{i}[i]
$$

is a silting object but not a bounded silting object. Since $\bigoplus_{i \in \mathbb{N}} P_{i}$ is a projective generator of $\mathcal{M}(\mathbb{K} Q)$ (see [31, Theorem 5.35]), it is clear that $M$ generates $\mathrm{D}(\mathcal{M}(\mathbb{K} Q))$. Furthermore, it is easy to see that $\operatorname{Hom}_{\mathrm{D}(\mathcal{M}(\mathbb{K} Q))}(M, M[i])=0$ for all $i>0$. It remains to check that $M^{\perp>0}$ is closed under coproducts in $\mathcal{M}(\mathbb{K} Q)$. First note that each $P_{i}$ is compact in $\mathrm{D}(\mathscr{A})$ (see also [47, Section 4.2]). Now, given a family $\left(X_{\lambda}\right)_{\lambda \in \Lambda}$ of objects in $M^{\perp>0}$, for any $k>0$, we have

$$
\operatorname{Hom}_{\mathrm{D}(\mathcal{M}(\mathbb{K} Q))}\left(M, \bigoplus_{\lambda \in \Lambda} X_{\lambda}[k]\right)=\prod_{i \in \mathbb{N}} \bigoplus_{\lambda \in \Lambda} \operatorname{Hom}_{\mathrm{D}(\mathcal{M}(\mathbb{K} Q))}\left(P_{i}[i], X_{\lambda}[k]\right)=0 .
$$

This shows that $M$ is a silting object in $\mathcal{M}(\mathbb{K} Q)$. Since $\mathcal{M}(\mathbb{K} Q)$ is a Grothendieck category, it follows from Remark 4.16(ii) that any bounded silting object in $\mathrm{D}(\mathcal{M}(\mathbb{K} Q))$ must lie in $\mathrm{D}^{b}(\mathcal{M}(\mathbb{K} Q))$. Hence $M$ is not a bounded silting object. 


\section{Derived equivalences}

In this section we will combine contents of Sects. 3 and 4 in order to discuss derived equivalences arising from realisation functors associated to tilting or cotilting t-structures. We will also reinterpret in terms of realisation functors a problem by Rickard on the shape of derived equivalences ([72]).

We will often consider the unbounded derived category of a heart of the form $\mathcal{H}_{M}$, for some silting or cosilting object $M$ in a triangulated category $\mathcal{T}$. No set-theoretical problems arise here, since from [73, Theorem 1], the category $\mathrm{D}\left(\mathcal{H}_{M}\right)$ exists (i.e. it has Hom-sets) when $\mathcal{H}_{M}$ has coproducts and enough projectives or when $\mathcal{H}_{M}$ has products and enough injectives. From Proposition 4.3 and Lemma 4.5, this includes the silting and cosilting cases, respectively, which are the focus of our approach.

In our discussion of derived equivalences, we will frequently interchange between considerations on bounded and unbounded derived categories. The reasons for this are already apparent in previous sections. While the unbounded derived category is a better setting for categorical constructions as it often admits products and coproducts (see also Sect. 6 for more advantages of working in the unbounded setting), it is in the bounded setting that we come across the current tools to build realisation functors. We believe that this obstacle can be overcome with a different approach to the construction of realisation functors, but this falls outside of the scope of this paper.

When realisation functors are considered with respect to filtered derived categories, we will omit in the notation of the functor the superscript referring to the f-category. Also for simplicity, the subscript of the functor indicative of a (silting) t-structure will be replaced by the silting or cosilting object that uniquely determines it. Here is an informal overview of this section.

\section{Subsection 5.1: Tilting and cotilting equivalences}

- We show that a realisation functor associated to a (co)silting t-structure is fully faithful if and only if it actually comes from a (co)tilting t-structure (Proposition 5.1).

- We state a derived Morita theory for abelian categories with a projective generator or an injective cogenerator (Theorem 5.3).

- We show the invariance of finite global dimension under tilting or cotilting derived equivalences of abelian categories.

\section{Subsection 5.2: Standard forms}

- We prove that the existence of a derived equivalence of standard type between $\mathbb{K}$-algebras (projective over a commutative ring $\mathbb{K}$ ) forces the associated realisation functor to be also an equivalence of standard type. Moreover, we provide an equivalent condition for a derived equivalence between such $\mathbb{K}$-algebras to be of standard type (Theorem 5.13).

- We show that Fourier-Mukai transforms in algebraic geometry are equivalent to some realisation functors (Proposition 5.17). In particular, we observe that Fourier-Mukai transforms can be thought of as cotilting equivalences.

\subsection{Tilting and cotilting equivalences}

We begin by discussing realisation functors associated to silting or cosilting objects. 
Proposition 5.1 Let $(X, \theta)$ be an $f$-category over a TR5 (respectively, TR5*) triangulated category $\mathcal{T}$. For a silting (respectively, cosilting) object $M$ in $\mathcal{T}$, the realisation functor real $\left.\right|_{M} ^{X}: \mathrm{D}^{b}\left(\mathcal{H}_{M}\right) \longrightarrow \mathcal{T}$ is fully faithful if and only if $M$ is a tilting (respectively, cotilting) object.

Proof We show the statement for silting/tilting objects, using the condition (Ef) of Theorem 3.11. The cosilting/cotilting case is entirely dual (using the condition (CoEf) of Theorem 3.11).

Let $M$ be a tilting object in $\mathcal{T}$. We only need to show that condition (Ef) holds for $\mathcal{H}_{M}$. Take $X$ and $Y$ in $\mathcal{H}_{M}$ and a morphism $g: X \longrightarrow Y[n]$ in $\mathcal{T}$, for some $n \geq 2$. By Proposition 4.3, $M$ is a generator in $\mathcal{H}_{M}$ and, thus, there is an epimorphism $h: M^{(I)} \longrightarrow X$ in $\mathcal{H}_{M}$, for some set $I$. Note that, $\mathcal{H}_{M}$ admits coproducts and that, since $\operatorname{Add}(M)$ is contained in the heart, coproducts of $M$ in $\mathcal{H}_{M}$ coincide with those in $\mathcal{T}$ (see Lemma 4.5). Since $Y$ lies in $M^{\perp>0}$, it is clear that $g \circ h=0$ and, thus, we have (Ef).

Conversely, suppose that real ${ }_{M}$ is fully faithful (i.e. we assume condition (Ef)). We show that, for any set $I, \tau_{M}^{\leq-1}\left(M^{(I)}\right)=0$, thus proving that $\operatorname{Add}(M)$ is contained in $\mathcal{H}_{M}$. Consider the canonical triangle

$$
\mathrm{H}_{M}^{0}\left(M^{(I)}\right)[-1] \longrightarrow \tau_{M}^{\leq-1} M^{(I)} \longrightarrow M^{(I)} \longrightarrow \mathrm{H}_{M}^{0}\left(M^{(I)}\right)
$$

and the canonical morphism $\tau_{M}^{\leq-1}\left(M^{(I)}\right) \longrightarrow \tau_{M}^{\geq-1} \tau_{M}^{\leq-1} M^{(I)}=\mathrm{H}_{M}^{-1}\left(M^{(I)}\right)[1]$. Let $g$ be the morphism between $\mathrm{H}_{M}^{0}\left(M^{(I)}\right)[-1]$ and $\mathrm{H}_{M}^{-1}\left(M^{(I)}\right)$ [1] obtained as the composition of the two morphisms above. Now, by condition (Ef), there is an object $C$ in $\mathcal{H}_{M}$ and an epimorphism $h: C \longrightarrow \mathrm{H}_{M}^{0}\left(M^{(I)}\right)$ such that the composition $g[1] \circ h: C \longrightarrow \mathrm{H}_{M}^{0}\left(M^{(I)}\right) \longrightarrow$ $\mathrm{H}_{M}^{-1}\left(M^{(I)}\right)[2]$ is zero. Now, the arguments in the proof of Proposition 4.3 can also show that $\mathrm{H}_{M}^{0}\left(M^{(I)}\right)$ is a projective object in $\mathcal{H}_{M}$ and, thus, the epimorphism $h$ splits in $\mathcal{H}_{M}$. This proves that $g[1]$ (and hence $g$ ) is the zero map. Since $\operatorname{Hom}_{\mathcal{T}}\left(M, \mathrm{H}_{M}^{-1}\left(M^{(I)}\right)[1]\right)=0$, we conclude that the canonical map $\tau_{M}^{\leq-1}\left(M^{(I)}\right) \longrightarrow \mathrm{H}_{M}^{-1}\left(M^{(I)}\right)$ [1] must also be the zero map and, therefore, $\mathrm{H}_{M}^{-1}\left(M^{(I)}\right)=0$ (see Sect. 2.1). This shows that $\tau_{M}^{\leq-2}\left(M^{(I)}\right) \cong \tau_{M}^{\leq-1}\left(M^{(I)}\right)$ and we can repeat the argument by considering the canonical map $\tau_{M}^{\leq-2}\left(M^{(I)}\right) \longrightarrow \mathrm{H}_{M}^{-2}\left(M^{(I)}\right)[2]$. It follows by induction that $\mathrm{H}_{M}^{i}\left(\tau_{\bar{M}}^{\leq-1}\left(M^{(I)}\right)\right)=0$ for all $i \leq-1$. Since the t-structure induced by $M$ is nondegenerate (see Remark 4.4), it follows that $\tau_{\bar{M}}^{\leq-1}\left(M^{(I)}\right)=0$ and, thus, $M^{(I)} \cong \mathrm{H}_{M}^{0}\left(M^{(I)}\right)$.

The following corollary is a non-compact analogue of [17, Theorem III.4.3].

Corollary 5.2 Let $\mathscr{A}$ be an abelian category such that $\mathrm{D}(\mathscr{A})$ is TR5 (respectively, TR5*) and let $M$ be a bounded silting (respectively, cosilting) object in $\mathrm{D}(\mathscr{A})$. Then the functor real ${ }_{M}: \mathrm{D}^{b}\left(\mathcal{H}_{M}\right) \longrightarrow \mathrm{D}(\mathscr{A})$ induces an equivalence between $\mathrm{D}^{b}\left(\mathcal{H}_{M}\right)$ and $\mathrm{D}^{b}(\mathscr{A})$ if and only if $M$ is tilting (respectively, cotilting).

Proof If $M$ is a bounded (co)silting object, then the essential image of real $M$ lies in $\mathrm{D}^{b}(\mathscr{A})$ (see Lemma 4.14) and the image coincides with $\mathrm{D}^{b}(\mathscr{A})$ whenever real ${ }_{M}$ is fully faithful (see Theorem 3.11). The result then follows from Proposition 5.1.

Given a bounded silting or cosilting object $M$ in the derived category of an abelian category, we will keep the notation real ${ }_{M}$ for the induced functor with codomain the bounded derived category. We are now able to discuss a derived Morita theory for abelian categories with a projective generator or an injective cogenerator (Theorem 5.3 in the introduction). The proof of the following theorem is a simple application of the above proposition and corollary. 
Theorem 5.3 Let $\mathscr{A}$ and $\mathscr{B}$ be abelian categories such that $\mathrm{D}(\mathscr{A})$ is TR5 (respectively, $\left.T R 5^{*}\right)$ and $\mathscr{B}$ has a projective generator (respectively, an injective cogenerator). Consider the following statements.

(i) There is a restrictable triangle equivalence $\Phi: \mathrm{D}(\mathscr{B}) \longrightarrow \mathrm{D}(\mathscr{A})$.

(ii) There is a bounded tilting (respectively, cotilting) object $M$ in $\mathrm{D}(\mathscr{A})$ such that $\mathcal{H}_{M} \cong \mathscr{B}$.

(iii) There is a triangle equivalence $\phi: \mathrm{D}^{b}(\mathscr{B}) \longrightarrow \mathrm{D}^{b}(\mathscr{A})$.

Then we have (i) $\Longrightarrow($ ii $) \Longrightarrow$ (iii). Moreover, if $\mathscr{B}$ has a projective generator and $\mathscr{A}=$ Mod- $R$, for a ring $R$, then we also have (iii) $\Longrightarrow$ (ii).

Proof Let $\mathscr{A}$ be such that $\mathrm{D}(\mathscr{A})$ is TR5 and assume that $\mathscr{B}$ has a projective generator $P$ (the proof for $\mathscr{B}$ with an injective cogenerator is entirely dual). By Lemma 4.10(iii), $P$ is a tilting object in $\mathrm{D}(\mathscr{B})$ and the associated tilting t-structure is the standard one. It is then clear that $P$ is a bounded tilting object.

(i) $\Longrightarrow$ (ii): Denote by $M$ the object $\Phi(P)$. Clearly, $M$ is a tilting object in $\mathrm{D}(\mathscr{A})$ (see Example 4.2(iii)) and the associated tilting t-structure is the image by $\Phi$ of the standard t-structure in $\mathrm{D}(\mathscr{B})$. Hence, we have $\mathcal{H}_{M} \cong \mathscr{B}$. Moreover, $M$ is a bounded tilting object since $\Phi$ is a restrictable equivalence (recall Definition 3.15).

(ii) $\Longrightarrow$ (iii): This follows from Proposition 5.1 and Corollary 5.2.

Suppose now that $\mathscr{A}=\operatorname{Mod}-R$, for a $\operatorname{ring} R$, and $\mathscr{B}$ has a projective generator.

(iii) $\Longrightarrow$ (ii): Denote by $M$ the object $\phi(P)$. It follows directly by the arguments in [71, Proposition 6.2] that $\phi$ induces an equivalence between $\mathrm{K}^{b}(\operatorname{Proj}-\mathscr{B})$ and $\mathrm{K}^{b}(\operatorname{Proj}-R)$. It is, however, clear that $\phi$ also induces an equivalence between $\mathrm{K}^{b}$ (Proj- $\left.\mathscr{B}\right)=$ $\mathrm{K}^{b}(\operatorname{Add}(P))$ and the smallest thick subcategory of $\mathrm{D}^{b}(R)$ containing $\operatorname{Add}(M)$ (denoted by thick $(\operatorname{Add}(M)))$. Therefore, we conclude that $M$ is an object of $\mathrm{D}^{b}(R)$ such that $\operatorname{Hom}_{\mathrm{D}^{b}(R)}\left(M, M^{(I)}[k]\right)=0$ for all $k \neq 0$ and thick $(\operatorname{Add}(M))=\mathrm{K}^{b}(\operatorname{Proj}-R)$. It then follows from [8, Proposition 4.2] that $M$ is indeed a tilting object in $\mathrm{D}(R)$. Finally, $M$ is a bounded tilting object as a consequence of Proposition 4.17.

Example 5.4 (i) Note that the equivalence between (ii) and (iii) in Theorem 5.3 recovers Rickard's derived Morita theory for rings ([71, Theorem 6.4]). For this purpose it is enough to recall from Corollary 4.7 that compact tilting objects yield hearts which are module categories.

(ii) Let $R$ be a ring and $T$ be a large $n$-tilting (respectively, $n$-cotilting) $R$-module (see Example 4.2(vi)). Note that by Proposition 4.17, $T$ is a bounded tilting (respectively, cotilting) object in $\mathrm{D}(R)$. Then, by Theorem 5.3 it follows that there is a triangle equivalence between $\mathrm{D}^{b}\left(\mathcal{H}_{T}\right)$ and $\mathrm{D}^{b}(R)$. In the n-cotilting case, this is a bounded version of [77, Theorem 5.21].

(iii) Since Grothendieck categories have injective cogenerators and their derived categories are TR5*, the above theorem covers a derived Morita theory for Grothendieck categories. Indeed, if the unbounded derived categories of two Grothendieck categories are equivalent via a restrictable equivalence, then one of them is the heart of a t-structure associated to a bounded cotilting object in the derived category of the other. Moreover, the realisation functor associated to this bounded cotilting object yields an equivalence of bounded derived categories.

Remark 5.5 Let $\mathscr{A}$ and $\mathscr{B}$ be as in Theorem 5.3.

(i) If $\mathscr{A}$ has exact coproducts, then there is a triangle equivalence $\phi: \mathrm{D}^{b}(\mathscr{B}) \longrightarrow \mathrm{D}^{b}(\mathscr{A})$ if and only if there is a tilting object $M$ in $\mathrm{D}^{b}(\mathscr{A})$ such that $\mathcal{H}_{M} \cong \mathscr{B}$. One direction is 
clear from the proof of (i) $\Longrightarrow$ (ii) above. For the converse, note that since $\mathscr{A}$ has exact coproducts, $\operatorname{Add}(M)$ is contained in $\mathrm{D}^{b}(\mathscr{A})$ and, therefore, the associated realisation functor yields an equivalence as in the proof of Proposition 5.1. The dual result follows analogously. Note, however, that the relation between (co)silting objects in $\mathrm{D}^{b}(\mathscr{A})$ and bounded silting objects in $\mathrm{D}(\mathscr{A})$ is not clear. The problem here lies in extending tstructures from bounded to unbounded derived categories.

(ii) As discussed at the end of Sect. 3, the question of whether (iii) implies (i) remains in general open. We will see, however, that in some cases we can guarantee the extendability of realisation functors (see Theorem 5.13 and Remark 5.14).

We now briefly discuss an application of the above theorem to representation theory of infinite quivers, proving a version of APR-tilting in this setting. Recall the notation set up before Example 4.18. The intuition from the theory of finite dimensional algebras leads us to think that the BGP-reflection functors on sources and sinks should provide derived equivalences. For infinite quivers, this cannot be achieved through the endomorphism ring of a tilting object (the reflected category cannot be regarded as a unital ring), but rather through the heart of a tilting object. We refer to [11] for a detailed discussion of reflection functors and derived equivalences in the setting of infinite quivers.

Let $Q$ be a quiver (possibly infinite) with no loops nor cycles. We assume that $Q$ is locally finite, i.e. that each vertex has only finitely many incoming and outgoing arrows. For a source $k$ in $Q$, define $\mu_{k}(Q)$ to be the quiver obtained from $Q$ by reversing the direction of every arrow starting in $k$ and keeping the remaining vertices and arrows as in $Q$. We show the following fact (compare with [11, Theorem 3.19]).

Proposition 5.6 If $k$ is a source of a locally finite quiver $Q$, then there is a triangle equivalence between $\mathrm{D}^{b}(\mathcal{M}(\mathbb{K} Q))$ and $\mathrm{D}^{b}\left(\mathcal{M}\left(\mathbb{K} \mu_{k}(Q)\right)\right)$.

Proof Let $Q_{0}$ be the set of vertices in $Q$ and let $I:=\left\{i \in Q_{0} \mid \operatorname{Hom}_{\mathbb{K} Q}(k, i) \neq 0\right\}$. Let $R_{k}$ denote the set of arrows from $k$ to some vertex in $I$. Since $Q$ is locally finite, $R_{k}$ is finite. For an arrow $\alpha$ in $\mathbb{K} Q$, denote by $t(\alpha)$ the target of $\alpha$. Consider the naturally induced map

$$
\phi: P_{k} \longrightarrow \bigoplus_{\alpha \in R_{k}} P_{t(\alpha)}
$$

and let $C$ denote its cokernel in $\mathcal{M}(\mathbb{K} Q)$. Note that $\phi$ is a left $\operatorname{Add}\left(\oplus_{j \neq k} P_{j}\right)$-approximation of $P_{k}$, i.e. any map from $P_{k}$ to an object in $\operatorname{Add}\left(\oplus_{j \neq k} P_{j}\right)$ must factor through $\phi$. We will check that $T:=C \oplus\left(\oplus_{j \neq k} P_{j}\right)$ is a bounded tilting object in $\mathrm{D}(\mathcal{M}(\mathbb{K} Q))$. Since $\phi$ is a monomorphism and the sum of all indecomposable projectives is a generator in $\mathrm{D}(\mathcal{M}(\mathbb{K} Q))$, it is easy to check that also $T$ is a generator of $\mathrm{D}(\mathcal{M}(\mathbb{K} Q))$. Since $T$ is a direct sum of finitely presented objects, it is clear that $T^{\perp>0}$ is closed under coproducts. Furthermore, since $T$ has projective dimension 1, it only remains to show that $\operatorname{Ext}_{\mathcal{N}(\mathbb{K} Q)}^{1}(T, T)=0$, i.e. to check that

$$
\operatorname{Ext}_{\mathcal{M}(\mathbb{K} Q)}^{1}(C, C)=0=\operatorname{Ext}_{\mathcal{M}(\mathbb{K} Q)}^{1}\left(C, \bigoplus_{j \neq k} P_{j}\right) .
$$

The first equality follows from applying $\operatorname{Hom}_{\mathcal{N}(\mathbb{K} Q)}(-, C)$ to the short exact sequence defined by $\phi$, using the projectivity of $P_{k}$ and the fact that $\phi$ is a left $\operatorname{Add}\left(\oplus_{j \neq k} P_{j}\right)$ approximation. The second one follows from applying $\operatorname{Hom}_{\mathcal{M}(\mathbb{K} Q)}\left(-, \oplus_{j \neq k} P_{j}\right)$ to the same sequence and using, once again, the approximation properties of $\phi$. It can also be checked that the object $T$ is a bounded tilting object (the associated t-structure is the HRS-tilt with 
respect to the torsion pair $\left(T^{\perp_{1}}, T^{\circ}\right)$ in $\mathcal{M}(\mathbb{K} Q)$-see Example 4.2(iv)). Then the realisation functor

$$
\operatorname{real}_{T}: \mathrm{D}^{b}\left(\mathcal{H}_{T}\right) \longrightarrow \mathrm{D}^{b}(\mathcal{M}(\mathbb{K} Q))
$$

is an equivalence. It remains to show that $\mathcal{M}\left(\mathbb{K} \mu_{k}(Q)\right)$ is equivalent to $\mathcal{H}_{T}$. An equivalence $\psi$ from $\mathbb{K} \mu_{k}(Q)$ to $\mathcal{H}_{T}$ can be defined by setting $\psi\left(P_{j}\right)=P_{j}$, for all $j \neq k$ and $\psi\left(P_{k}\right)=C$. By definition of $C$, the Hom-spaces are preserved and $\psi$ extends to the whole category since it is defined on a projective generator. Since $T$ is a projective generator in the heart, the functor so defined is an equivalence, as wanted.

Theorem 5.3 leads us to discuss a derived invariant which is well-understood for rings: the finiteness of global dimension. This invariant generalises to the setting of abelian categories with a projective generator or an injective cogenerator. Recall that an abelian category $\mathscr{A}$ has finite global dimension if there is a positive integer $n$ such that the Yoneda Ext functor $\operatorname{Ext}_{\mathscr{A}}^{n}(-,-)$ is identically zero. Whenever $\mathscr{A}$ has a projective generator or an injective cogenerator, the following is a well-known lemma, which we prove for convenience of the reader.

Lemma 5.7 Let $\mathscr{A}$ be a cocomplete (respectively, complete) abelian category with a projective generator $P$ (respectively, an injective cogenerator $E$ ). The following statements are equivalent.

(i) $\mathscr{A}$ has finite global dimension;

(ii) The smallest thick subcategory of $\mathrm{D}(\mathscr{A})$ containing $\operatorname{Add}(P)$ (respectively, $\operatorname{Prod}(E))$ is $\mathrm{D}^{b}(\mathscr{A})$.

Proof We discuss the case of $\mathscr{A}$ with a projective generator; the injective cogenerator case is dual.

(i) $\Longrightarrow$ (ii): Note that $\mathrm{D}^{b}(\mathscr{A})$ is the smallest thick subcategory containing $\mathscr{A}$. So it suffices to show that any object in $\mathscr{A}$ lies in the smallest thick subcategory containing $\operatorname{Add}(P)$, which is $\mathrm{K}^{b}(\operatorname{Add}(P))$. Let $X$ be an object of $\mathscr{A}$ and consider a projective resolution of $X:\left(Q^{i}, d^{i}\right)_{i \leq 0}$. Let $f$ be an epimorphism $P^{(I)} \rightarrow$ Ker $d^{-n}$, yielding an exact sequence of projective objects

$$
P^{(I)} \stackrel{f}{\longrightarrow} Q^{-n} \stackrel{d^{-n}}{\longrightarrow} \cdots \longrightarrow Q^{0} \stackrel{d^{0}}{\longrightarrow} X
$$

which must then split at some point by (1). Thus, $X$ admits a finite projective resolution, as wanted.

(ii) $\Longrightarrow($ i $):$ Since $\mathrm{K}^{b}(\operatorname{Add}(P))=\mathrm{D}^{b}(\mathscr{A})$, it follows that every object of $\mathscr{A}$ admits a finite projective resolution. Since Yoneda Ext-groups can be computed by projective resolutions in the first component (see, for example, [36, III.6.14]) it only remains to show that there is a uniform choice of integer $n$ for all objects in $\mathscr{A}$. Suppose that this is not the case, i.e. that for any $n$ in $\mathbb{N}$, there is an object $X_{n}$ in $\mathscr{A}$ with projective dimension greater or equal than $n$. Since $\mathscr{A}$ is cocomplete, considering the coproduct of the family $\left(X_{n}\right)_{n \in \mathbb{N}}$ would yield an object of infinite projective dimension, contradicting our assumption.

Proposition 5.8 Let $\mathscr{A}$ be an abelian category with a projective generator (respectively, injective cogenerator) and suppose that $\mathrm{D}(\mathscr{A})$ is TR5 (respectively, TR5*). Let $\mathbb{T}$ be a $t$ structure in $\mathrm{D}(\mathscr{A})$ satsifying the equivalent conditions of Lemma 4.14 and suppose that the realisation functor real $\mathbb{T}_{\mathbb{T}}: \mathrm{D}^{b}(\mathcal{H}(\mathbb{T})) \longrightarrow \mathrm{D}(\mathscr{A})$ is fully faithful. 
(i) If $\mathscr{A}$ has finite global dimension, then so does $\mathcal{H}(\mathbb{T})$.

(ii) If $\mathbb{T}=\left(M^{\perp>0}, M^{\perp<0}\right)$ for a bounded tilting (respectively, cotilting) object $M$ in $\mathrm{D}(\mathscr{A})$, then the following are equivalent:

(a) $\mathscr{A}$ has finite global dimension;

(b) $\mathcal{H}_{M}$ has finite global dimension;

(c) The smallest thick subcategory of $\mathrm{D}(\mathscr{A})$ containing $\operatorname{Add}(M)($ respectively, $\operatorname{Prod}(M))$ is $\mathrm{D}^{b}(\mathscr{A})$.

Proof First note that if $\mathrm{D}(\mathscr{A})$ is TR5 (respectively, TR5*), then $\mathscr{A}$ is cocomplete (respectively, complete), by Lemma 4.5. Assume now that $\mathrm{D}(\mathscr{A})$ is TR5 and that $\mathscr{A}$ has a projective generator $P$ (the other statement follows by dualising the arguments).

(i) Since $\mathbb{T}$ satisfies the equivalent conditions of Lemma 4.14, one may consider the induced functor real $\mathbb{T}_{\mathbb{T}}: \mathrm{D}^{b}(\mathcal{H}(\mathbb{T})) \longrightarrow \mathrm{D}^{b}(\mathscr{A})$ which is, by assumption, a triangle equivalence (see also Theorem 3.11). Since $\mathcal{H}(\mathbb{T})$ lies in $\mathrm{D}^{b}(\mathscr{A})$ and $\mathscr{A}$ has finite global dimension, $\mathcal{H}(\mathbb{T})$ is contained in the smallest thick subcategory generated by $P$, i.e. in $\mathrm{K}^{b}(\operatorname{Add}(P)$ ) (see Lemma 5.7). Hence, for any objects $X$ and $Y$ in $\mathcal{H}(\mathbb{T}), \operatorname{Ext}_{\mathcal{H}(\mathbb{T})}^{n}(X, Y) \cong \operatorname{Hom}_{\mathrm{D}(\mathscr{A})}(X, Y[n])$ must vanish for $n \gg 0$ (as before, we identify $X$ and $Y$ with their images under real $\left.\right|_{\mathbb{T}}$, since this functor acts as the identity on $\mathcal{H}(\mathbb{T})$ by definition).

Suppose now that $\mathcal{H}(\mathbb{T})$ does not have finite global dimension, i.e. that there are sequences of objects $\left(X_{i}\right)_{i \in \mathbb{N}}$ and $\left(Y_{i}\right)_{i \in \mathbb{N}}$ in $\mathcal{H}(\mathbb{T})$ such that Ext $\underset{\mathcal{H}(\mathbb{T})}{\geq i}\left(X_{i}, Y_{i}\right) \neq 0$. By Lemma 4.5, the heart $\mathcal{H}(\mathbb{T})$ is a cocomplete abelian category and the coproducts in $\mathcal{H}(\mathbb{T})$, here denoted with the symbol $\oplus$, are computed by using the truncation $\tau_{\mathbb{T}}^{\geq 0}$ on the coproduct available in $\mathrm{D}(\mathscr{A})$, here denoted with the symbol $\amalg$. We will show that $\operatorname{Ext}_{\mathcal{H}(\mathbb{T})}^{\geq n}\left(\bigoplus_{i \in \mathbb{N}} X_{i}, \bigoplus_{i \in \mathbb{N}} Y_{i}\right) \neq 0$, for all $n \in \mathbb{N}$, thus reaching a contradiction with the previous paragraph. Note that, since $\tau_{\mathbb{T}}^{\geq 0}$ is left adjoint to the inclusion of the coaisle in $\mathrm{D}(\mathscr{A})$, we have that

$$
\begin{aligned}
& \operatorname{Ext}_{\mathcal{H}(\mathbb{T})}^{\geq n}\left(\bigoplus_{i \in \mathbb{N}} X_{i}, \bigoplus_{i \in \mathbb{N}} Y_{i}\right) \cong \operatorname{Hom}_{\mathrm{D}(\mathscr{A})}\left(\coprod_{i \in \mathbb{N}} X_{i}, \bigoplus_{i \in \mathbb{N}} Y_{i}[\geq n]\right) \\
& \cong \prod_{i \in \mathbb{N}} \operatorname{Hom}_{\mathrm{D}(\mathscr{A})}\left(X_{i}, \bigoplus_{i \in \mathbb{N}} Y_{i}[\geq n]\right)
\end{aligned}
$$

for any $n$ in $\mathbb{N}$. The heart $\mathcal{H}(\mathbb{T})$ admits finite products and they coincide with finite coproducts-and these biproducts indeed coincide with those of $\mathrm{D}(\mathscr{A})$ (since both $\mathbb{T} \leq 0$ and $\mathbb{T}^{\geq 0}$ are closed under finite biproducts). Thus, if we write, for some integer $n$,

$$
\bigoplus_{i \in \mathbb{N}} Y_{i}=Y_{n} \times \bigoplus_{i \in \mathbb{N} \backslash\{n\}} Y_{i}
$$

since $\operatorname{Hom}_{\mathrm{D}(\mathscr{A})}\left(X_{n},-[\geq n]\right)$ commutes with products and since by assumption $\operatorname{Hom}_{\mathrm{D}(\mathscr{A})}\left(X_{n}, Y_{n}[\geq n]\right) \neq 0$, we get that $\operatorname{Hom}_{\mathrm{D}(\mathscr{A})}\left(X_{n}, \bigoplus_{i \in \mathbb{N}} Y_{i}[\geq n]\right) \neq 0$.

(ii) (a) $\Longrightarrow$ (b): This follows directly from (i).

(b) $\Longrightarrow\left(\right.$ a): Let $N=$ real $_{M}^{-1}(P)$. Then $N$ is a tilting object in $\mathrm{D}\left(\mathcal{H}_{M}\right)$ whose heart is equivalent to $\mathscr{A}$. Since, by assumption, $\mathcal{H}_{M}$ has finite global dimension, we can apply the statement (a) $\Longrightarrow$ (b) exchanging the roles of $\mathscr{A}$ and $\mathcal{H}_{M}$.

(b) $\Longleftrightarrow(c)$ : This statement follows from a combined application of (i) and Lemma 5.7, since $M$ is a projective generator of $\mathcal{H}_{M}$ (see Proposition 4.3). 


\subsection{Standard forms}

So far, our discussion of derived equivalences has mostly been concerned with their existence. We would like now to discuss their shape, i.e. their explicit description as functors. Our approach is in part motivated by Proposition 3.17. In the context of derived equivalences of rings, this problem was addressed in [72] for algebras over a commutative ring $\mathbb{K}$, which are projective as $\mathbb{K}$-modules (for simplicity, we will call such algebras projective $\mathbb{K}$-algebras). Therein, a partial answer to the problem is presented through the concept of equivalences of standard type. In this subsection we will often refer to tilting complexes in the original version of the concept, as defined in [71]. Note, however, that these are precisely the compact tilting objects in the derived category of a ring, following Definition 4.1.

Remark 5.9 As seen in Corollary 4.7, given a compact silting object $M$, there is an equivalence of abelian categories $\operatorname{Hom}_{\mathcal{H}_{M}}\left(\mathrm{H}_{M}^{0}(M),-\right): \mathcal{H}_{M} \longrightarrow \operatorname{Mod}-E d_{\mathcal{T}}\left(\mathrm{H}_{M}^{0}(M)\right)$. In what follows, we identify these two categories without mention to the equivalence functor. In particular, given a tilting complex $T$ in the derived category of a ring $A$, the realisation functor real $_{T}$ will be regarded, via this identification, as a functor $\mathrm{D}^{b}\left(\operatorname{End}_{\mathrm{D}(A)}(T)\right) \longrightarrow \mathrm{D}^{b}(A)$.

Definition 5.10 Let $\mathbb{K}$ be a commutative ring and let $A$ and $B$ be projective $\mathbb{K}$-algebras. We say that an equivalence $\phi: \mathrm{D}^{b}(B) \longrightarrow \mathrm{D}^{b}(A)$ is of standard type if there is a complex of $B$-A-bimodules such that $\phi$ is naturally equivalent to $-\otimes_{B}^{\mathbb{L}} X$. Such an object $X$ is called a two-sided tilting complex.

Example 5.11 Let $\mathbb{K}$ be a commutative ring and $A$ and $B$ two projective $\mathbb{K}$-algebras. Any exact equivalence $F:$ Mod- $B \longrightarrow \operatorname{Mod}-A$ is a tensor product with a bimodule. Hence, its derived functor is a standard equivalence of derived categories.

The two-sided tilting complex $X$ in the above definition, when seen as an object in $\mathrm{D}^{b}(A)$, is a tilting complex $T:=X_{A}$. It is known that, in the derived category of $B$ - $A$-bimodules, $X$ can be chosen such that both $X_{A}$ and ${ }_{B} X$ are complexes of projective modules and such that $X_{A}$ is still isomorphic to $T$ in $\mathrm{D}^{b}(A)$ ([81, Proposition 6.4.4]). Moreover, Rickard proved in [72] the following result on the existence of equivalences of standard type (see also [46, Theorem]).

Theorem 5.12 [72, Corollary 3.5] Let $\mathbb{K}$ be a commutative ring and $A$ and $B$ two projective $\mathbb{K}$-algebras. If $\mathrm{D}^{b}(B)$ is triangle equivalent to $\mathrm{D}^{b}(A)$, then there is a two-sided tilting complex ${ }_{B} X_{A}$ and a derived equivalence of standard type $-\otimes_{B}^{\mathbb{L}} X: \mathrm{D}^{b}(B) \longrightarrow \mathrm{D}^{b}(A)$.

Motivated by Proposition 3.17, we have the following result (Theorem B in the introduction).

Theorem 5.13 Let $\mathbb{K}$ be a commutative ring, $A$ and $B$ projective $\mathbb{K}$-algebras and $T$ a compact tilting object in $\mathrm{D}(A)$ such that $\mathrm{End}_{\mathrm{D}(A)}(T) \cong B$. Then the functor real $_{T}$ is an equivalence of standard type. Moreover, a triangle equivalence $\psi: \mathrm{D}^{b}(B) \longrightarrow \mathrm{D}^{b}(A)$ is of standard type if and only if $\psi$ admits an f-lifting $\Psi: \mathrm{DF}^{b}(B) \longrightarrow \mathrm{DF}^{b}(A)$ to the filtered bounded derived categories.

Proof Since $T$ is a compact tilting object with endomorphism ring $B$, it follows from classical derived Morita theory [71] that $\mathrm{D}^{b}(B)$ is equivalent to $\mathrm{D}^{b}(A)$. From Theorem 5.12, there is a two-sided tilting complex ${ }_{B} X_{A}$ (such that $X_{A} \cong T$ ) and an equivalence of standard type 
$\phi:=-\otimes_{B}^{\mathbb{L}} X: \mathrm{D}^{b}(B) \longrightarrow \mathrm{D}^{b}(A)$. We first show that $\phi$ admits an f-lifting to the filtered bounded derived categories and then argue with Theorem 3.13 to prove that real ${ }_{T}$ is of standard type.

As mentioned above, the two-sided tilting complex $X$ can be chosen such that ${ }_{B} X$ is a complex of projective $B$-modules. It is then easy to check that given a monomorphism $f: Y \longrightarrow Z$ in the category of complexes $C^{b}(B), f \otimes_{B} X$ is still a monomorphism. Then, for an object $(Y, F)$ in $\mathrm{CF}^{b}(B)$, there is an obvious filtration $F \otimes_{B} X$ on the complex $Y \otimes_{B} X$ induced by $F$, i.e. $\left(F \otimes_{B} X\right)_{n} Y \otimes_{B} X:=F_{n} Y \otimes_{B} X$. It follows that there is a functor $\psi: \mathrm{CF}^{b}(B) \longrightarrow \mathrm{CF}^{b}(A)$ such that $\psi(Y, F)=\left(Y \otimes_{B}\right.$ $\left.X, F \otimes_{B} X\right)$. Again, since $-\otimes_{B} X$ is an exact functor between the categories of complexes, the functor $\psi$ sends filtered quasi-isomorphisms in $\mathrm{CF}^{b}(B)$ to filtered quasiisomorphisms in $\mathrm{CF}^{b}(A)$, thus inducing a functor $\Phi: \mathrm{DF}^{b}(B) \longrightarrow \mathrm{DF}^{b}(A)$. The functor $\Phi$ is clearly an f-functor and it is an f-lifting of $\phi$ to the filtered bounded derived categories.

Now, from Theorem 3.13 and Proposition 3.14, it follows that $\phi \cong \operatorname{real}_{T} \circ \mathrm{D}^{b}\left(\phi^{0}\right)$, where $\phi^{0}$ is the induced exact functor of abelian categories $\phi^{0}$ : Mod- $B \longrightarrow \phi(\operatorname{Mod}-B)$. Identifying $\phi(\operatorname{Mod}-B)$ with a module category Mod- $B^{\prime}$, Example 5.11 yields that $\mathrm{D}^{b}\left(\phi^{0}\right)$ is a derived equivalence of standard type. Since the inverse of a derived equivalence of standard type and the composition of two derived equivalences of stardard type are both of standard type ([72, Proposition 4.1]) the claim follows.

It remains to show that any triangle equivalence $\psi: D^{b}(B) \longrightarrow D^{b}(A)$ admitting an f-lifting to the filtered bounded derived categories is of standard type. Indeed, under this assumption, from Theorem 3.13 and Proposition 3.14, we have that $\psi \cong \operatorname{real}_{\psi(B)} \circ \mathrm{D}^{b}\left(\psi^{0}\right)$. Since, as shown in the above paragraphs, both $\operatorname{real}_{\psi(B)}$ and $\mathrm{D}^{b}\left(\psi^{0}\right)$ are equivalences of standard type, it follows from [72, Proposition 4.1] that the composition $\psi$ is of standard type.

Remark 5.14 Note that if the conditions in the above theorem are satisfied, then real $\mathrm{I}_{T}$ is in fact an extendable equivalence (see Definition 3.15). This follows from the fact that derived equivalences of standard type are extendable [47].

There is another setting where a suitable notion of standard derived equivalences exists: derived categories of coherent sheaves. We recall the definition of a Fourier-Mukai transform. For the rest of this section, let $\mathbb{K}$ be an algebraically closed field of characteristic zero. Given a smooth projective variety $X$ over $\mathbb{K}$, we denote by $\mathrm{D}^{b}(\operatorname{coh}(X))$ the bounded derived category of coherent sheaves over $X$.

Definition 5.15 Let $X$ and $Y$ be smooth projective varieties over $\mathbb{K}$ and let $q: X \times Y \longrightarrow X$ and $p: X \times Y \longrightarrow Y$ be the natural projections. For any object $P$ in $\mathrm{D}^{b}(\operatorname{coh}(X \times Y))$ we define the Fourier-Mukai transform with kernel $\mathbf{P}$ as the functor $\Theta_{P}: \mathrm{D}^{b}(\operatorname{coh}(X)) \longrightarrow$ $\mathrm{D}^{b}(\operatorname{coh}(Y))$ which sends an object $Z$ to $\theta_{P}(Z):=\mathbb{R} p_{*}\left(q^{*} Z \otimes_{X \times Y}^{\mathbb{L}} P\right)$, where $p_{*}$ and $q^{*}$ represent, respectively, the (left exact) direct image and the (exact) pullback functors defined on coherent sheaves.

Note that the functor $q^{*}$ is exact since $q$ is a flat morphism (see also [41, Chapter 5]). The following theorem of Orlov provides a standard form for equivalences between derived categories of coherent sheaves.

Theorem 5.16 [66] Let $X$ and $Y$ be two smooth projective varieties over $\mathbb{K}$. Any fully faithful triangle functor $F: \mathrm{D}^{b}(\operatorname{coh}(X)) \longrightarrow \mathrm{D}^{b}(\operatorname{coh}(Y))$ admiting left and right adjoints is 
naturally equivalent to a Fourier-Mukai transform $\Theta_{P}$, for an object $P$ in $\mathrm{D}^{b}(\operatorname{coh}(X \times Y))$, unique up to isomorphism.

Proposition 5.17 Let $\theta_{P}: \mathrm{D}^{b}(\operatorname{coh}(X)) \longrightarrow \mathrm{D}^{b}(\operatorname{coh}(Y))$ be a Fourier-Mukai transform between two smooth projective varieties $X$ and $Y$ over $\mathbb{K}$. Then $\theta_{P}$ is naturally equivalent to real $\left.\right|_{\mathbb{T}} \circ \mathbb{D}^{b}\left(\theta_{P}^{0}\right)$, where $\mathbb{T}=\left(\theta_{P}(\mathbb{D} \leq 0), \theta_{P}(\mathbb{D} \geq 0)\right)$.

Proof The technique employed here is analogous to the ones before. First we observe that $\theta_{P}$ lifts to the filtered bounded derived categories of $X$ and $Y$. This follows from the fact that $\theta_{P}$ is the composition of functors that admit f-liftings. In fact, since $\mathbb{R} p_{*}$ and $q^{*}$ are derived functors of functors between abelian categories, if follows that they admit f-liftings to the filtered bounded derived categories of coherent sheaves of $X$ and $Y$ (see [15, Example A2]). Moreover, also the derived tensor functor admits an f-lifting. Indeed, since $X$ is projective and smooth, any object in $\mathrm{D}^{b}(\operatorname{coh}(X))$ is isomorphic to a perfect complex, i.e. to a complex of locally free sheaves. In particular, we may assume without loss of generality that $P$ is such a complex. It is then easy to check that tensoring with $P$ is an exact functor in the category of complexes of coherent sheaves and, hence, it induces a functor between categories of filtered complexes. The argument then follows analogously to the proof of Theorem 5.13, using Theorem 3.13 and Proposition 3.14.

Finally, we remark that the $\mathrm{t}$-structure $\mathbb{T}$ in the above proposition is, in fact, the restriction to $\mathrm{D}^{b}(\operatorname{coh}(X))$ of a cotilting t-structure in $\mathrm{D}(\mathrm{Qcoh}(X))$, the unbounded derived category of quasicoherent sheaves over $X$. The functor $\theta_{P}$ can be extended to a functor $\Theta_{P}: \mathrm{D}(\mathrm{Q} \operatorname{coh}(X)) \longrightarrow \mathrm{D}(\mathrm{Q} \operatorname{coh}(Y))$, by using the same formula and this extension still has both left and right adjoints (which are again Fourier-Mukai transforms, see [59] and [10, Section 2.2 and 3.1]). Hence, $\Theta_{P}$ preserves coproducts. Since the varieties are smooth, the compact objects in $\mathrm{D}(\mathrm{Q} \operatorname{coh}(X))$ are precisely those in $\mathrm{D}^{b}(\operatorname{coh}(X))$. Thus, $\Theta_{P}$ is a coproduct preserving triangle functor between compactly generated triangulated categories which restricts to an equivalence on compact objects. By [75, Lemma 3.3], this means that also $\Theta_{P}$ is a triangle equivalence. If $E$ is an injective cogenerator of Qcoh $(X)$, then the t-structure $\mathbb{T}$ in the proof of the above proposition is the restriction to $\mathrm{D}^{b}(\operatorname{coh}(X))$ of the t-structure associated to the cotilting object $\Theta_{P}(E)$. Informally, one may then say that Fourier-Mukai transforms are cotilting equivalences.

\section{Recollements of derived categories}

Let $R$ be a ring and $e$ an idempotent element of $R$. As observed in [27], the recollement of the module category Mod- $R$ induced by the idempotent element $e$ (see Example 2.5) always induces a recollement of triangulated categories $\mathrm{R}_{\mathrm{tr}}(\operatorname{Ker} \mathrm{D}(e(-)), \mathrm{D}(\operatorname{Mod}-R), \mathrm{D}(\mathrm{Mod}-$ $e R e)$ ), where $\mathrm{D}(e(-)): \mathrm{D}(\operatorname{Mod}-R) \longrightarrow \mathrm{D}(\operatorname{Mod}-e R e)$ is the derived functor induced by the exact functor $e(-):$ Mod- $R \longrightarrow$ Mod- $e$ Re. It also follows from [27] that $\operatorname{Ker} \mathrm{D}(e(-))$ is triangle equivalent with $\mathrm{D}(\operatorname{Mod}-R / \operatorname{Re} R)$ if and only if the natural map $R \longrightarrow R / R e R$ is a homological ring epimorphism. In this section we generalise this result, investigating when do recollements of abelian categories $\mathrm{R}_{\mathrm{ab}}(\mathscr{B}, \mathscr{A}, \mathscr{C})$ induce recollements of the associated unbounded derived categories $\mathrm{R}_{\mathrm{tr}}(\mathrm{D}(\mathscr{B}), \mathrm{D}(\mathscr{A}), \mathrm{D}(\mathscr{C}))$. Moreover, we will discuss when is a recollement of derived categories equivalent to a derived version of a recollement of abelian categories. This is intimately related with glueing tilting objects. Here is an informal overview of this section. 


\section{Subsection 6.1: Homological embeddings}

- We prove in a rather general context that fully faithful functors between abelian categories preserving Yoneda extensions yield fully faithful derived functors between unbounded derived categories (Theorem 6.9).

- We present equivalent conditions for a recollement of abelian categories to induce a recollement of unbounded derived categories, generalising the analogous result for rings in [27] (Theorem 6.10).

\section{Subsection 6.2: Glueing tilting and equivalences of recollements}

- Under some technical assumptions, we give an equivalent condition (in terms of glueing tilting t-structures) for a recollement of derived categories to be equivalent to the derived version of a recollement of abelian categories (Theorem 6.13).

- We specialise Theorem 6.13 to recollements of derived categories of certain $\mathbb{K}$-algebras, where all the technical conditions are automatically satisfied (Corollary 6.14). This statement reflects the main idea of this section, relating equivalences of recollements with glueing of tilting t-structures.

- We apply the above results to recollements of derived module categories of finite dimensional hereditary $\mathbb{K}$-algebras, over a field $\mathbb{K}$, answering a question posed by $\mathrm{Xi}$ (Theorem 6.17).

\subsection{Homological embeddings}

In this section we will study exact fully faithful functors between abelian categories whose derived functors are fully faithful. Examples of these are well-known in representation theory: a ring epimorphism induces a fully faithful restriction of scalars functor, and its derived functor is fully faithful if and only if some homological conditions are satisfied (see Theorem 2.4).

To build recollements of triangulated categories, we will often need to ensure the existence of adjoint pairs. A powerful tool for this purpose is Brown representability. Recall that a TR5 triangulated category $\mathcal{T}$ satisfies Brown representability if every cohomological functor $H: \mathcal{T}^{o p} \longrightarrow$ Mod- $\mathbb{Z}$ which sends coproducts to products is representable (i.e. $H \cong$ $\operatorname{Hom}_{\mathcal{T}}(-, X)$ for some $X$ in $\left.\mathcal{T}\right)$.

Theorem 6.1 [62, Theorem 8.4.4] Let $\mathcal{T}$ and $\mathcal{V}$ be TR5 triangulated categories and suppose that $\mathcal{T}$ satisfies Brown representability. Then any coproduct-preserving functor $F: \mathcal{T} \longrightarrow \mathcal{V}$ has a right adjoint.

There are many examples of triangulated categories satisfying Brown representability, in particular some derived categories of abelian categories. The following concept will be used to provide examples (we refer to [48, Section 2] for the terminology Milnor colimit and Milnor limit).

Definition 6.2 The derived category $\mathrm{D}(\mathscr{A})$ of an abelian category $\mathscr{A}$ is left-complete if $\mathrm{D}(\mathscr{A})$ is TR5* and any object $X$ is isomorphic to a Milnor limit of its standard truncations $\left(\tau^{\geq n} X\right)_{n \leq 0}$ with respect to the canonical maps $\tau^{\geq n-1} X \longrightarrow \tau^{\geq n} X$, for $n \leq 0$.

Remark 6.3 (i) We can dually define the notion of a right-complete derived category. It is, however, easy to see that any TR5 derived category $\mathrm{D}(\mathscr{A})$ is right-complete. This 
follows from [60, Remark 24 and Lemma 64(iii)] since, for any complex $X$, there is a quasi-isomorphism in $\mathrm{K}(\mathscr{A})$ between the Milnor colimit of $\left(\tau^{\leq n} X\right)_{n \geq 0}$ and the direct limit of the same system in $\mathrm{C}(\mathscr{A})$ (which is isomorphic to $X$ ).

(ii) Note that right and left-completeness can analogously be defined in any triangulated category $\mathcal{T}$ endowed with a t-structure $\mathbb{T}$, in which case we say that $\mathcal{T}$ is right or leftcomplete with respect to $\mathbb{T}$. Given a triangle equivalence $\phi: \mathrm{D}(\mathscr{A}) \longrightarrow \mathrm{D}(\mathscr{B})$, then $\mathrm{D}(\mathscr{A})$ is right or left-complete if and only if $\mathrm{D}(\mathscr{B})$ is left or right-complete with respect to the t-structure $\phi\left(\mathbb{D}_{\mathscr{A}}\right)=\left(\phi\left(\mathbb{D}_{\mathscr{A}}^{\leq 0}\right), \phi\left(\mathbb{D}_{\mathscr{A}}^{\geq 0}\right)\right)$.

Example 6.4 Let $\mathscr{A}$ be an abelian category.

(i) If $\mathscr{A}$ has a projective generator and $\mathrm{D}(\mathscr{A})$ is TR5*, then $\mathrm{D}(\mathscr{A})$ is left-complete. In fact, it follows from [60, Remark 27 and Lemma 67(i)] that, for any complex $X$, there is a quasi-isomorphism in $\mathrm{K}(\mathscr{A})$ between the inverse limit of $\left(\tau^{\geq n} X\right)_{n \leq 0}$ in the category of complexes (which is isomorphic to $X$ ) and the Milnor limit of the same system.

(ii) In particular, as a consequence of Lemma 4.5(i) and 4.3, if $\mathrm{D}(\mathscr{A})$ is TR5 (respectively, TR5*) and $M$ is a silting object in $\mathrm{D}(\mathscr{A})$, then $\mathrm{D}\left(\mathcal{H}_{M}\right)$ is right-complete (respectively, left-complete).

(iii) Assume that $\mathscr{A}$ has an injective cogenerator and finite global dimension. If $\mathrm{D}(\mathscr{A})$ is TR5 $*$ and $M$ is a cotilting object in $\mathrm{D}(\mathscr{A})$, then $\mathrm{D}\left(\mathcal{H}_{M}\right)$ is left-complete (in particular, so is $\mathrm{D}(\mathscr{A})$ ). This follows from $\mathcal{H}_{M}$ having finite global dimension (Proposition 5.8(ii)) and from [39, Theorem 1.3].

(iv) If $\mathscr{A}$ is a Grothendieck category, then $\mathrm{D}(\mathscr{A})$ is right-complete but not always leftcomplete [63]. However, from item (ii) above, $\mathrm{D}(R)$ is left-complete for any ring $R$, and from [39, Remark 3.3], D $(\mathrm{Qcoh}(X))$ is left-complete for any separated quasicompact scheme $X$.

The next theorem recalls two examples of categories satisfying Brown representability.

Theorem 6.5 Let $\mathscr{A}$ be an abelian category.

(i) [33, Theorem 3.1] If $\mathscr{A}$ is Grothendieck, then $\mathrm{D}(\mathscr{A})$ satisfies Brown representability.

(ii) [58, Theorem 1.1] If $\mathscr{A}$ has an injective cogenerator and $\mathrm{D}(\mathscr{A})$ is left-complete, then $\mathrm{D}(\mathscr{A})^{\text {op }}$ satisfies Brown representability.

Given a TR5 (respectively, TR5*) triangulated category, we say that a triangulated subcategory is localising (respectively, colocalising) if it is coproduct-closed (respectively, product-closed), i.e. if the inclusion functor commutes with coproducts (respectively, products). The following result is well-known, still we present a proof for convenience of the reader.

Lemma 6.6 Let $\mathscr{A}$ be a Grothendieck abelian category.

(i) [33, Proposition 3.3] If $X$ is a localising subcategory of $D(\mathscr{A})$ and $\mathscr{A} \subseteq X$, then $x=D(\mathscr{A})$.

(ii) Assume that $\mathrm{D}(\mathscr{A})$ is left-complete. If $y$ is a colocalising subcategory of $D(\mathscr{A})$ and $\mathscr{A} \subseteq \mathrm{y}$, then $\mathrm{y}=D(\mathscr{A})$.

Proof (i) This statement can also be shown using [2, Theorem 5.7]. Let $G$ be a generator of $\mathscr{A}$. It suffices to prove that the smallest localising subcategory $\mathcal{L}$ of $\mathrm{D}(\mathscr{A})$ containing $G$ is the whole derived category. By [2, Theorem 5.7], the inclusion of $\mathcal{L}$ in $D(\mathscr{A})$ has a right adjoint and, thus, the pair $\left(\mathcal{L}, \mathcal{L}^{\perp}\right)$ is a (stable) t-structure in $\mathrm{D}(\mathscr{A})$. It remains to show that $\mathcal{L}^{\perp}=0$. Now, if $X$ lies in $\mathcal{L}^{\perp}$, then we have $\operatorname{Hom}_{\mathrm{D}(\mathscr{A})}(G, X[i])=0$ for all $i$ in $\mathbb{Z}$, which from Lemma 4.10(i) implies that $X=0$. 
(ii) This proof is essentially contained in [18, Applications 2.4 and 2.4']. We prove that the smallest colocalising subcategory $\mathcal{C}$ containing the injective objects of $\mathscr{A}$ is $D(\mathscr{A})$. Clearly any bounded complex of injective objects lies in $\mathcal{C}$. Now every bounded below complex of injective objects lies in $\mathcal{C}$ is an inverse limit of bounded complexes in the category $\mathrm{C}(\mathscr{A})$, where the maps in the inverse system are identities componentwise. This implies that every bounded below complex of injective objects is a Milnor limit of bounded ones (see for example [60, Proposition 85]) and, therefore, every such complex lies in $\mathcal{C}$. Finally, since every bounded below complex in $\mathrm{D}(\mathscr{A})$ is quasi-isomorphic to a bounded below complex of injective objects, and since $\mathrm{D}(\mathscr{A})$ is left-complete, we conclude that $\mathrm{C}=\mathrm{D}(\mathscr{A})$.

Remark 6.7 By [62, Proposition 8.4.6], a triangulated category satisfying Brown representability is TR5*. Hence, as mentioned before, the derived category of a Grothendieck category is TR5*. Moreover, from [39, Theorem 1.3], if products in a Grothendieck category $\mathscr{A}$ have finite homological dimension (i.e. the $n$-th right derived functor vanishes for some $n>0$ ), then $\mathrm{D}(\mathscr{A})$ is left-complete. In particular, this is satisfied if the Grothendieck category has finite global dimension (see also Example 6.4).

We now recall the definition of a homological embedding of abelian categories (see [69]).

Definition 6.8 An exact functor $i: \mathscr{B} \longrightarrow \mathscr{A}$ between abelian categories is called a homological embedding, if the map $i_{X, Y}^{n}: \operatorname{Ext}_{\mathscr{B}}^{n}(X, Y) \longrightarrow \operatorname{Ext}_{\mathscr{A}}^{n}(i(X), i(Y))$ is an isomorphism of the abelian groups of Yoneda extensions, for all $X, Y$ in $\mathscr{B}$ and for all $n \geq 0$.

As mentioned earlier, homological ring epimorphisms are examples of homological embeddings. In that case, by Theorem 2.4, the derived functor $f_{*}: \mathrm{D}(B) \longrightarrow \mathrm{D}(A)$ is fully faithful. Our next theorem generalises this statement for homological embeddings $i: \mathscr{B} \longrightarrow \mathscr{A}$ of abelian categories. Note that we do not assume $\mathscr{B}$ to be a Serre subcategory of $\mathscr{A}$ (compare [80, Theorem 2.1] and [39, Theorem 1.5]).

Theorem 6.9 Let $\mathscr{A}$ be an abelian category such that $\mathrm{D}(\mathscr{A})$ is TR5 and TR5* and let $\mathscr{B}$ be a Grothendieck category such that $\mathrm{D}(\mathscr{B})$ is left-complete. If $i: \mathscr{B} \longrightarrow \mathscr{A}$ is an exact full embedding such that the derived functor $i: \mathrm{D}(\mathscr{B}) \longrightarrow \mathrm{D}(\mathscr{A})$ preserves products and coproducts, then the following statements are equivalent.

(i) The functor $i: \mathscr{B} \longrightarrow \mathscr{A}$ is a homological embedding.

(ii) The derived functor $i: D(\mathscr{B}) \longrightarrow D(\mathscr{A})$ is fully faithful.

If, in addition, $\mathrm{D}(\mathscr{A})$ is left-complete, then the statements (i) and (ii) are also equivalent to:

(iii) The derived functor $i$ induces a triangle equivalence between $\mathrm{D}(\mathscr{B})$ and the full subcategory $\mathrm{D}_{\mathscr{B}}(\mathscr{A})$ of $\mathrm{D}(\mathscr{A})$, whose objects are complexes of objects in $\mathscr{A}$ with cohomologies in $i(\mathscr{B})$.

Proof (i) $\Longrightarrow$ (ii): Let $Y \in \mathrm{D}(\mathscr{B})$. We define the following full subcategory of $\mathrm{D}(\mathscr{B})$ :

$$
\mathcal{M}_{Y}=\left\{X \in \mathrm{D}(\mathscr{B}) \mid \alpha_{X, Y}: \operatorname{Hom}_{\mathrm{D}(\mathscr{B})}(X[k], Y) \stackrel{\cong}{\longrightarrow} \operatorname{Hom}_{\mathrm{D}(\mathscr{A})}(i(X[k]), i(Y)), \forall k \in \mathbb{Z}\right\}
$$

where $\alpha_{X, Y}$ is the natural map of abelian groups induced by the functor $i: \mathrm{D}(\mathscr{B}) \longrightarrow \mathrm{D}(\mathscr{A})$. We show that $\mathcal{M}_{Y}$ is a localising subcategory of $\mathrm{D}(\mathscr{B})$. First, it is clear that $\mathcal{M}_{Y}$ is closed under shifts. For $K$ and $M$ in $\mathcal{M}_{Y}$ and a triangle $\Delta$ in $\mathrm{D}(\mathscr{B})$ of the form

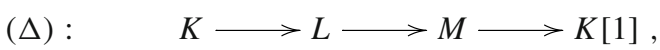


consider the triangle $i(\Delta)$ in $\mathrm{D}(\mathscr{A})$. Applying to $\Delta$ and $i(\Delta)$ the cohomological functors $\operatorname{Hom}_{\mathrm{D}(\mathscr{B})}(-, Y)$ and $\operatorname{Hom}_{\mathrm{D}(\mathscr{A})}(-, i(Y))$, respectively, we obtain a commutative diagram with exact rows as follows:

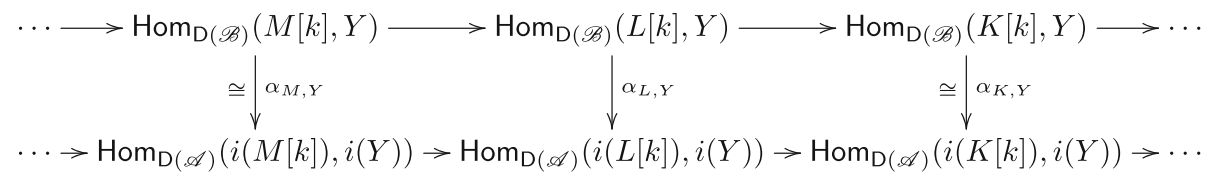

Hence, the map $\alpha_{L, Y}$ is an isomorphism and the object $L$ lies in $\mathcal{M}_{Y}$. Let $\left(X_{i}\right)_{i \in I}$, be a family of objects in $\mathcal{M}_{Y}$. We show that $\bigsqcup_{i \in I} X_{i}$ lies in $\mathcal{M}_{Y}$, concluding that $\mathcal{M}_{Y}$ is localising in $\mathscr{B}$. Since, by assumption, the derived functor $i: \mathrm{D}(\mathscr{B}) \longrightarrow \mathrm{D}(\mathscr{A})$ preserves coproducts, the following diagram commutes.

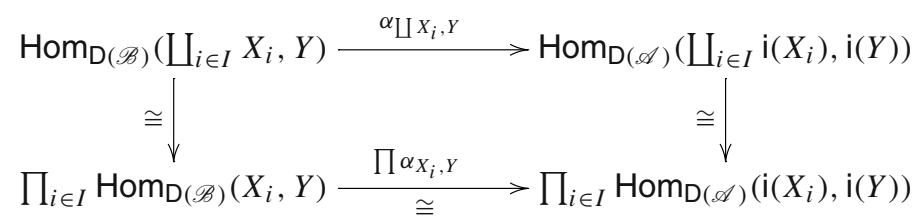

Then the map $\alpha_{\amalg X_{i}, Y}$ is an isomorphism and $\mathcal{M}_{Y}$ is a localising subcategory of $\mathrm{D}(\mathscr{B})$. In particular, for every $B$ in $\mathscr{B}$ the subcategory $\mathcal{M}_{B}$ is localising in $\mathrm{D}(\mathscr{B})$ and $\mathscr{B} \subseteq \mathcal{M}_{B}$ since the functor $i: \mathscr{B} \longrightarrow \mathscr{A}$ is a homological embedding. Then from Lemma 6.6(i) we get that $\mathcal{M}_{B}=\mathrm{D}(\mathscr{B})$ for every $B$ in $\mathscr{B}$.

Let $X$ be an object in $\mathrm{D}(\mathscr{B})$ and consider the full subcategory of $\mathrm{D}(\mathscr{B})$ :

$$
{ }_{X} \mathcal{M}=\left\{Y \in \mathrm{D}(\mathscr{B}) \mid \alpha_{X, Y}: \operatorname{Hom}_{\mathrm{D}(\mathscr{B})}(X, Y[k]) \stackrel{\cong}{\longrightarrow} \operatorname{Hom}_{\mathrm{D}(\mathscr{A})}(i(X), i(Y[k])), \forall k \in \mathbb{Z}\right\} .
$$

Then, dually to the argument above, it follows that ${ }_{x} \mathcal{M}$ is a colocalising subcategory of $\mathrm{D}(\mathscr{B})$. Since, for any $B$ in $\mathscr{B}$ we have $\mathcal{M}_{B}=\mathrm{D}(\mathscr{B})$ it follows that $\mathscr{B} \subseteq{ }_{X} \mathcal{M}$ for any $X$ in $\mathrm{D}(\mathscr{B})$. Since $\mathrm{D}(\mathscr{B})$ is left-complete, Lemma 6.6(ii) shows that ${ }_{X} \mathcal{M}=\mathrm{D}(\mathscr{B})$. Hence, the derived functor $i: \mathrm{D}(\mathscr{B}) \longrightarrow \mathrm{D}(\mathscr{A})$ is fully faithful.

(ii) $\Longrightarrow($ i): Suppose that $i: \mathrm{D}(\mathscr{B}) \longrightarrow \mathrm{D}(\mathscr{A})$ is fully faithful and let $X$ and $Y$ be objects in $\mathscr{B}$ and $n \geq 0$. Then we have the following chain of natural isomorphisms:

$$
\operatorname{Ext}_{\mathscr{B}}^{n}(X, Y) \cong \operatorname{Hom}_{\mathrm{D}(\mathscr{B})}(X, Y[n]) \cong \operatorname{Hom}_{\mathrm{D}(\mathscr{A})}(i(X), i(Y)[n]) \cong \operatorname{Ext}_{\mathscr{A}}^{n}(i(X), i(Y))
$$

thus showing that the functor $i: \mathscr{A} \longrightarrow \mathscr{B}$ is a homological embedding.

Assume now that also $\mathrm{D}(\mathscr{A})$ is left-complete. Note that it is also right-complete by Remark 6.3(i).

(iii) $\Longrightarrow$ (ii): This is clear.

(ii) $\Longrightarrow$ (iii): Assume that the derived functor $i$ is fully faithful, i.e. that $i$ induces a triangle equivalence between $\mathrm{D}(\mathscr{B})$ and $X:=\operatorname{Im} i$. It is clear that $X$ is a full extension-closed subcategory of $\mathrm{D}_{\mathscr{B}}(\mathscr{A})$. First observe that $i(\mathscr{B})$ lies in $\mathcal{X}$ and, hence, so does $\mathrm{D}_{\mathscr{B}}^{b}(\mathscr{A})$, since every object in $\mathrm{D}_{\mathscr{B}}^{b}(\mathscr{A})$ can be obtained as a finite extension of objects in $i(\mathscr{B})$. Since $\mathrm{D}(\mathscr{A})$ is both left and right-complete, every object in $\mathrm{D}_{\mathscr{B}}(\mathscr{A}), X$ can be expressed as a Milnor limit of a Milnor colimit of bounded complexes with cohomologies in $i(\mathscr{B})$ (since standard truncations respect the cohomologies). Since $\mathrm{D}(\mathscr{B})$ is TR5 and TR5* and $i$ preserves products and coproducts, $X$ is closed under Milnor limits and Milnor colimits, thus finishing the proof. 
Motivated by Example 2.5, the following result gives necessary and sufficient conditions for certain recollements of abelian categories to induce recollements of unbounded derived categories. The key ingredient of the proof is the equivalence between $\mathrm{D}_{\mathscr{B}}(\mathscr{A})$ and $\mathrm{D}(\mathscr{B})$ established in Theorem 6.9.

Theorem 6.10 Let $R_{\mathrm{ab}}(\mathscr{B}, \mathscr{A}, \mathscr{C})$ be a recollement of abelian categories as in (2.3). Suppose that $\mathscr{B}$ is a Grothendieck category such that $\mathrm{D}(\mathscr{B})$ is left-complete and suppose that $\mathrm{D}(\mathscr{A})$ is TR5 and left-complete. The following statements are equivalent.

(i) The functor $i_{*}: \mathscr{B} \longrightarrow \mathscr{A}$ is a homological embedding and $i_{*}: \mathrm{D}(\mathscr{B}) \longrightarrow \mathrm{D}(\mathscr{A})$ commutes with products and coproducts.

(ii) There is a recollement of triangulated categories

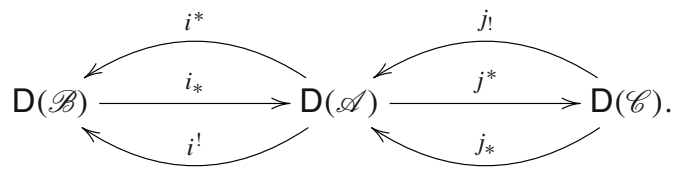

Proof (i) $\Longrightarrow$ (ii): From the recollement $\mathrm{R}_{\mathrm{ab}}(\mathscr{B}, \mathscr{A}, \mathscr{C})$ we have the exact sequence of abelian categories $0 \longrightarrow \mathscr{B} \longrightarrow \mathscr{A} \longrightarrow \mathscr{C} \longrightarrow 0$. Since the quotient functor $\mathscr{A} \longrightarrow \mathscr{C}$ has a right (or left) adjoint, it follows that $0 \longrightarrow \mathrm{D}_{\mathscr{B}}(\mathscr{A}) \longrightarrow \mathrm{D}(\mathscr{A}) \longrightarrow \mathrm{D}(\mathscr{C}) \longrightarrow 0$ is an exact sequence of triangulated categories, where $\mathrm{D}_{\mathscr{B}}(\mathscr{A})$ is the full subcategory of $\mathrm{D}(\mathscr{A})$ consisting of complexes whose cohomology lie in $i_{*}(\mathscr{B})$ (see, for instance, [53, Lemma 5.9]). The functor $i_{*}: \mathscr{B} \longrightarrow \mathscr{A}$ is a homological embedding, thus from Theorem 6.9 the derived functor $i_{*}: \mathrm{D}(\mathscr{B}) \longrightarrow \mathrm{D}(\mathscr{A})$ is fully faithful and $\mathrm{D}_{\mathscr{B}}(\mathscr{A})$ is equivalent to $\mathrm{D}(\mathscr{B})$. This implies that $0 \longrightarrow \mathrm{D}(\mathscr{B}) \longrightarrow \mathrm{D}(\mathscr{A}) \longrightarrow \mathrm{D}(\mathscr{C}) \longrightarrow 0$ is an exact sequence of triangulated categories. Since both $\mathrm{D}(\mathscr{B})$ and $\mathrm{D}(\mathscr{B})^{o p}$ satisfy Brown representability (Theorem 6.5) and $i_{*}$ preserves both products and coproducts, it follows from Theorem 6.1 that $i_{*}: \mathrm{D}(\mathscr{B}) \longrightarrow \mathrm{D}(\mathscr{A})$ has both a left and a right adjoint. On the other hand, by [26, Theorem 2.1], we obtain that the derived functor $j^{*}: \mathrm{D}(\mathscr{A}) \longrightarrow \mathrm{D}(\mathscr{C})$ admits a left and right adjoint and, therefore, we get a recollement of triangulated categories as wanted.

(ii) $\Longrightarrow(\mathrm{i})$ : Since $(\mathrm{D}(\mathscr{B}), \mathrm{D}(\mathscr{A}), \mathrm{D}(\mathscr{C}))$ is a recollement, the functor $i_{*}: \mathrm{D}(\mathscr{B}) \longrightarrow \mathrm{D}(\mathscr{A})$ is fully faithful. Then, by Theorem 6.9 we infer that $i_{*}: \mathscr{B} \longrightarrow \mathscr{A}$ is a homological embedding. Since the abelian category $\mathscr{B}$ is Grothendieck, it follows that the derived category $\mathrm{D}(\mathscr{B})$ is TR5 and TR5*. Hence, the functor $i_{*}: \mathrm{D}(\mathscr{B}) \longrightarrow \mathrm{D}(\mathscr{A})$ preserves coproducts and products since it is both a left and a right adjoint.

Remark 6.11 Note that, in the above theorem, we do not describe explicitly how to obtain the adjoints of $i_{*}$ and $j^{*}$ in the recollement of derived categories. If, however, we assume some further conditions for the abelian categories $\mathscr{A}$ and $\mathscr{C}$, we can say more about these functors. In particular:

- If $\mathscr{A}$ is Grothendieck, then $i^{!}$is the right derived functor of the right adjoint of the inclusion functor $\mathscr{B} \longrightarrow \mathscr{A}$. If, furthermore, $\mathscr{A}$ has enough projectives, then $i^{*}$ is the left derived functor of the left adjoint of the inclusion functor $\mathscr{B} \longrightarrow \mathscr{A}$.

- If $\mathscr{C}$ is Grothendieck, then $j_{*}$ is the right derived functor of the right adjoint of the quotient functor $\mathscr{A} \longrightarrow \mathscr{C}$. If, furthermore, $\mathscr{C}$ has enough projectives, then $j$ ! is the left derived functor of the left adjoint of the quotient functor $\mathscr{A} \longrightarrow \mathscr{C}$.

Note also that, as a consequence of Theorem 6.10, we obtain the result of [27] for recollements of derived module categories, already mentioned in Example 2.5. More concretely, 
we have that given a ring $A$ and an idempotent element $e$ of $A$, the ring epimorphism $f: A \longrightarrow A / A e A$ is homological if and only if there is a recollement of triangulated categories of the form (2.1).

\subsection{Glueing tilting and equivalences of recollements}

Our aim in this subsection is to identify which recollements of derived categories are equivalent to derived versions of recollements of abelian categories. We will provide an answer to this question in terms of the glueing of (co)tilting t-structures.

We begin with two useful properties of derived recollements. On one hand, we discuss the glueing of standard t-structures along such a recollement, motivating a necessary condition towards our answer (Theorem 6.13) to the proposed problem. On the other hand, we restate in this context the exact sequence (3.1) of f-categories (see Proposition 3.8) for filtered derived categories, which we use to prove a corollary of the main theorem (Corollary 6.15).

Lemma 6.12 Let $R_{\mathrm{ab}}(\mathscr{B}, \mathscr{A}, \mathscr{C})$ be a recollement of abelian categories as in (2.3). Suppose that $\mathscr{B}$ is a Grothendieck category such that $\mathrm{D}(\mathscr{B})$ is left-complete and suppose that $\mathrm{D}(\mathscr{A})$ is TR5 and left-complete. If the functor $i_{*}: \mathscr{B} \longrightarrow \mathscr{A}$ is a homological embedding and $i_{*}: \mathrm{D}(\mathscr{B}) \longrightarrow \mathrm{D}(\mathscr{A})$ commutes with products and coproducts, then the following statements hold.

(i) The standard t-structures in $\mathrm{D}(\mathscr{B})$ and $\mathrm{D}(\mathscr{C})$ glue to the standard $t$-structure in $\mathrm{D}(\mathscr{A})$ along the recollement (6.1).

(ii) The f-categories over $\mathrm{D}(\mathscr{B})$ and $\mathrm{D}(\mathscr{C})$ induced by the filtered derived category $\mathrm{DF}(\mathscr{A})$ over $\mathrm{D}(\mathscr{A})$ coincide, respectively, with the filtered derived categories $\mathrm{DF}(\mathscr{B})$ and $\mathrm{DF}(\mathscr{C})$. In particular, there is an exact sequence of filtered derived categories:

$$
0 \longrightarrow \mathrm{DF}(\mathscr{B}) \longrightarrow \mathrm{DF}(\mathscr{A}) \longrightarrow \mathrm{DF}(\mathscr{C}) \longrightarrow 0 \text {. }
$$

Proof (i) From Theorem 6.10 we get a recollement of derived categories $\mathrm{R}_{\operatorname{tr}}(\mathrm{D}(\mathscr{B}), \mathrm{D}(\mathscr{A})$, $\mathrm{D}(\mathscr{B})$ ), see diagram (6.1). Since $i_{*}: \mathrm{D}(\mathscr{B}) \longrightarrow \mathrm{D}(\mathscr{A})$ and $j^{*}: \mathrm{D}(\mathscr{A}) \longrightarrow \mathrm{D}(\mathscr{C})$ are the derived functors of the underlying exact functors $i_{*}: \mathscr{B} \longrightarrow \mathscr{A}$ and $j^{*}: \mathscr{A} \longrightarrow \mathscr{C}$, respectively, it follows that the derived functors $i_{*}$ and $j^{*}$ are t-exact with respect to the standard t-structures. Hence, the triangle functor $i^{*}: \mathrm{D}(\mathscr{A}) \longrightarrow \mathrm{D}(\mathscr{B})$ in the recollement diagram (6.1) is right t-exact with respect to the standard t-structure. Then clearly we have $\mathbb{D}_{\mathscr{A}}^{\leq 0} \subseteq\left\{X \in \mathbb{D}(\mathscr{A}) \mid j^{*}(X) \in \mathbb{D}_{\mathscr{C}}^{\leq 0}\right.$ and $\left.i^{*}(X) \in \mathbb{D}_{\mathscr{B}}^{\leq 0}\right\}$. Similarly, we get that $\mathbb{D}_{\mathscr{A}}^{\geq 0} \subseteq\left\{X \in \mathrm{D}(\mathscr{A}) \mid j^{*}(X) \in \mathbb{D}_{\mathscr{C}}^{\geq 0}\right.$ and $\left.i^{!}(X) \in \mathbb{D}_{\mathscr{B}}^{\geq 0}\right\}$. We infer that these t-structures coincide.

(ii) From Theorem 6.10, there is an exact sequence of derived categories

$$
0 \longrightarrow \mathrm{D}(\mathscr{B}) \stackrel{i_{*}}{\longrightarrow} \mathrm{D}(\mathscr{A}) \stackrel{j^{*}}{\longrightarrow} \mathrm{D}(\mathscr{C}) \longrightarrow 0 .
$$

For an object $(X, F)$ in $\operatorname{DF}(\mathscr{B})$, where $F$ is a finite filtration of $X$, it follows from the exactness of $i_{*}: \mathscr{B} \longrightarrow \mathscr{A}$ that $i_{*}(X)$ has an induced filtration $i_{*}(F)$ and $\left(i_{*}(X), i_{*}(F)\right)$ lies in $\operatorname{DF}(\mathscr{A})$. Also, it is easy to see that $\operatorname{gr}_{i_{*}(F)}^{n}\left(i_{*}(X)\right)=i_{*}\left(\operatorname{gr}_{F}^{n}(X)\right)$ and, thus, it lies in $i_{*}(\mathrm{D}(\mathscr{B}))$. Hence, $i_{*}$ induces a functor

$$
i_{*}^{\mathrm{F}}: \mathrm{DF}(\mathscr{B}) \longrightarrow y:=\left\{(X, F) \in \operatorname{DF}(\mathscr{A}) \mid \operatorname{gr}_{F}^{n}(X) \in i_{*}(\mathrm{D}(\mathscr{B})) \text { for all } n \in \mathbb{Z}\right\}
$$

and one can check, using the exactness of $i_{*}: \mathscr{B} \longrightarrow \mathscr{A}$, that $i_{*}^{\mathrm{F}}$ is an f-functor (see Definition 3.5). 
We show that $i_{*}^{\mathrm{F}}$ is an equivalence. An easy induction argument shows that $i_{*}^{\mathrm{F}}$ is essentially surjective. In fact, since $i_{*}$ is a fully faithful triangle functor, $i_{*}(\mathrm{D}(\mathscr{B}))$ is a triangulated subcategory. Hence, given $(Z, L)$ in $y$, each $L_{n} Z$ lies in $i_{*}(\mathrm{D}(\mathscr{B}))$, since $L_{n} Z$ is a finite extension of its $\mathrm{gr}_{L}^{k}$-components, with $k \geq n$. From the fact that $i_{*}: \mathscr{B} \longrightarrow \mathscr{A}$ induces a fully faithful exact functor between the categories of complexes, it then follows that $(Z, L)$ can be identified with an object in $\operatorname{DF}(\mathscr{B})$. We show that $i_{*}^{\mathrm{F}}$ is faithful. Let $f:(X, F) \longrightarrow(Y, G)$ be a morphism in $\operatorname{DF}(\mathscr{B})$ such that $i_{*}^{\mathrm{F}}(f)=0$. Let $f$ be represented by a roof:

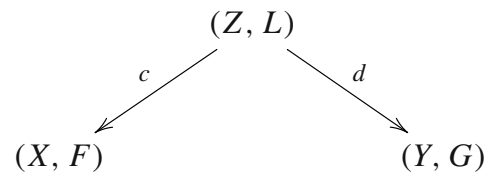

where $c$ is a filtered quasi-isomorphism in $\operatorname{KF}(\mathscr{B})$. It is easy to see that the morphism $f=d \circ c^{-1}$ can be written as a sequence of roofs $\left(\ldots, L_{a} d \circ\left(L_{a} c\right)^{-1}, L_{a+1} d \circ\right.$ $\left.\left(L_{a+1} c\right)^{-1}, \ldots\right)$ in $\mathrm{K}(\mathscr{B})$ (see also Example 3.2). Hence, it follows from the faithfulness of $i_{*}: \mathrm{D}(\mathscr{B}) \longrightarrow \mathrm{D}(\mathscr{A})$ that $f=0$. It remains to show that $i_{*}^{\mathrm{F}}$ is full. Let $f: i_{*}^{\mathrm{F}}(X, F) \longrightarrow i_{*}^{\mathrm{F}}(Y, G)$ be a morphism in $y$. The map $f$ can be represented by a roof similar to the one above, where the maps $c$ and $d$ now lie in $\operatorname{KF}(\mathscr{A})$. We claim that the middle object $(Z, L)$ lies in the image of the functor $i_{*}^{\mathrm{F}}$. The map $c$ is a filtered quasi-isomorphism, i.e, for all $k$ in $\mathbb{Z}$ the maps $L_{k} c: L_{k} Z \longrightarrow\left(i_{*} F\right)_{k} i_{*}(X)$ are quasi-isomorphisms in $\mathrm{K}(\mathscr{A})$. Equivalently, the complexes $\operatorname{gr}_{L}(Z)$ and $\operatorname{gr}_{i_{*} F}\left(i_{*} X\right)$ are quasi-isomorphic ([42, V.1.2]) and, thus, $(Z, L)$ also lies in the image of $i_{*}^{\mathrm{F}}$. Writing $f$ as a sequence of roofs as before, the fullness of $i_{*}: \mathrm{D}(\mathscr{B}) \longrightarrow \mathrm{D}(\mathscr{A})$ guarantees that each component of $f$ lies in $i_{*}(\mathrm{D}(\mathscr{B}))$. Finally, it can be checked (using the faithfulness of $i_{*}: \mathrm{D}(\mathscr{B}) \longrightarrow \mathrm{D}(\mathscr{A})$ ) that the preimages of each component of $f$ under $i_{*}$ form a compatible sequence of morphisms in $\mathrm{D}(\mathscr{B})$, i.e. there is a morphism in $\operatorname{DF}(\mathscr{B})$ which is a preimage of $f$ under $i_{*}^{\mathrm{F}}$.

We now show that the quotient f-category $\operatorname{DF}(\mathscr{A}) / y$ is equivalent to $\operatorname{DF}(\mathscr{C})$. The exact functor $j^{*}: \mathscr{A} \longrightarrow \mathscr{C}$ clearly defines a functor $j_{\mathrm{F}}^{*}: \operatorname{DF}(\mathscr{A}) \longrightarrow \operatorname{DF}(\mathscr{C})$ which factors uniquely through $\operatorname{DF}(\mathscr{A}) / y$. Hence, we get a functor $\phi: \operatorname{DF}(\mathscr{A}) / y \longrightarrow \operatorname{DF}(\mathscr{C})$. Conversely, since $j_{*}: \mathscr{C} \longrightarrow \mathscr{A}$ is left exact, it induces a functor $\operatorname{KF}(\mathscr{C}) \longrightarrow \operatorname{KF}(\mathscr{A})$, which we also denote by $j_{*}$. Consider the composition

$$
\operatorname{KF}(\mathscr{C}) \stackrel{j_{*}}{\longrightarrow} \operatorname{KF}(\mathscr{A}) \longrightarrow \mathrm{DF}(\mathscr{A}) \longrightarrow \mathrm{DF}(\mathscr{A}) / y
$$

where the last two functors are the obvious localisation functors. We claim that it sends filtered acyclic complexes to zero, hence yielding a functor $\psi: \operatorname{DF}(\mathscr{C}) \longrightarrow \operatorname{DF}(\mathscr{A}) / y$. Let $\left(j_{*}(X), j_{*}(F)\right)$ be an object in $\operatorname{DF}(\mathscr{A})$ where $(X, F)$ is acyclic in $\operatorname{KF}(\mathscr{C})$. It suffices to show that $\operatorname{gr}_{j_{*}(F)}^{n}\left(j_{*}(X)\right)$ lies in $i_{*}(\mathrm{D}(\mathscr{B}))$ for all integers $n$. From the exact sequence (6.2) this is equivalent to show that $j^{*}\left(\operatorname{gr}_{j_{*}(F)}^{n}\left(j_{*}(X)\right)\right)=0$ for all integers $n$. Using the adjoint pair $\left(j^{*}, j_{*}\right)$ at the level of homotopy categories and since the counit $j^{*} j_{*} \longrightarrow \operatorname{Id}_{\mathrm{K}(\mathscr{C})}$ is a natural equivalence we derive that $j^{*}\left(\operatorname{gr}_{j_{*}(F)}^{n}\left(j_{*}(X)\right)\right) \cong \operatorname{gr}_{F}^{n}(X)$ which is acyclic, thus zero in $\mathrm{D}(\mathscr{C})$, proving the claim. Finally, using the counit of the adjunction induced by $\left(j^{*}, j_{*}\right)$ at the level of filtered complexes, one can check that $\phi$ and $\psi$ are quasi-inverse f-functors, thus finishing the proof.

We now prove the main theorem of this section. 
Theorem 6.13 Let $\mathscr{A}, \mathscr{B}$ and $\mathscr{C}$ be abelian categories whose derived categories are TR5 and TR5*. Suppose furthermore that $\mathscr{B}$ is a Grothendieck category. Let $\mathcal{R}$ be a recollement of the form

$\mathcal{R}:$

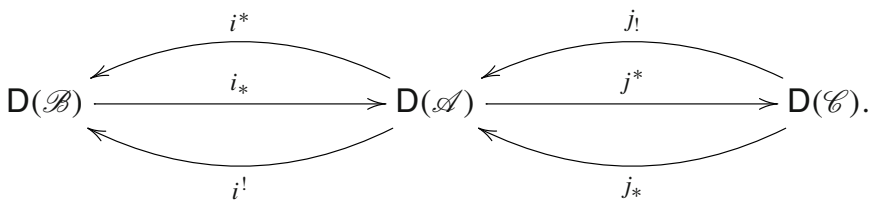

Then the following statements are equivalent.

(i) There are abelian categories $\mathcal{U}, \mathcal{V}$ and $\mathcal{W}$ with a projective generator (respectively, an injective cogenerator) and a recollement of abelian categories

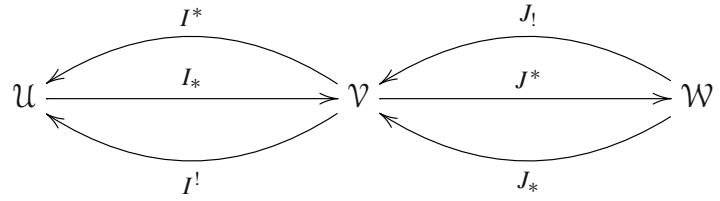

such that

- $\mathcal{U}$ is a Grothendieck category and $\mathrm{D}(\mathrm{U})$ is left-complete;

- the derived category $\mathrm{D}(\mathcal{V})$ is TR5 and left-complete;

- $I_{*}$ is a homological embedding and $I_{*}: \mathrm{D}(\mathcal{U}) \longrightarrow \mathrm{D}(\mathcal{V})$ preserve products and coproducts;

- the associated derived recollement is equivalent to $\mathcal{R}$ via restrictable equivalences.

(ii) There are bounded tilting (respectively, cotilting) objects $V$ in $\mathrm{D}(\mathscr{A}), U$ in $\mathrm{D}(\mathscr{B})$ and $W$ in $\mathrm{D}(\mathscr{C})$ such that

- $\mathcal{H}_{U}$ is a Grothendieck category and $\mathrm{D}\left(\mathcal{H}_{U}\right)$ is left-complete;

- the derived category $\mathrm{D}\left(\mathcal{H}_{V}\right)$ is left-complete;

- there is an f-category $(X, \theta)$ over $\mathrm{D}(\mathscr{A})$ such that the realisation functor real $\left.\right|_{V} ^{X}$ is an extendable equivalence;

- the associated tilting t-structures in $\mathrm{D}(\mathscr{B})$ and $\mathrm{D}(\mathscr{C})$ glue along $\mathcal{R}$ to the associated tilting $t$-structure in $\mathrm{D}(\mathscr{A})$.

Proof Once again, the tilting and cotilting cases are dual to each other. We prove the tilting case.

(i) $\Longrightarrow$ (ii): From Theorem 6.10, we conclude that the recollement of abelian categories $\mathrm{R}_{\mathrm{ab}}(\mathcal{U}, \mathcal{V}, \mathcal{W})$ can be derived. By assumption, there is an equivalence of recollements as follows: 


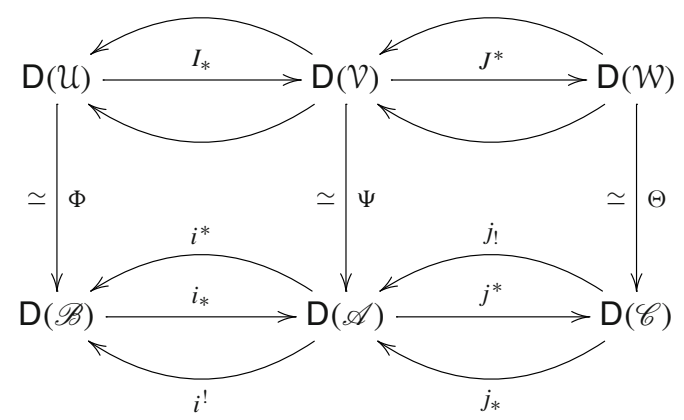

where $\Phi, \Psi$ and $\Theta$ are restrictable equivalences. Therefore, by Theorem 5.3, there are bounded tilting objects $V, U$ and $W$ in $\mathrm{D}(\mathscr{A}), \mathrm{D}(\mathscr{B})$ and $\mathrm{D}(\mathscr{C})$, respectively, such that $\mathcal{H}_{V} \cong \mathcal{V}$, $\mathcal{H}_{U} \cong \mathcal{U}$ and $\mathcal{H}_{W} \cong \mathcal{W}$.

Since the top recollement is derived from an abelian recollement, it follows from Lemma 6.12(i) that the standard t-structures in $\mathrm{D}(\mathcal{U})$ and $\mathrm{D}(\mathcal{W})$ glue to the standard $\mathrm{t}$ structure in $\mathrm{D}(\mathcal{W})$. Furthermore, the standard t-structures in the top recollement are sent to the the tilting t-structures generated by $V, U$ and $W$ in the bottom recollement. Hence, it follows from the commutativity of the diagram (6.4) that the glueing of the t-structures generated by $U$ and $W$ is the t-structure generated by $V$. The assumption that $\mathcal{U}$ is Grothendieck then translates into the fact that $\mathcal{H}_{U}$ is Grothendieck (since they are equivalent abelian categories). Also the left-completeness properties required in (i) imply the left-completeness properties of (ii). Finally, observe from Proposition 3.17(ii) that there is a choice of an f-category $(X, \theta)$ over $\mathrm{D}(\mathscr{A})$ such that $\Psi^{b} \cong \operatorname{real}_{V}^{X} \circ \mathrm{D}^{b}\left(\Psi^{0}\right)$, where $\Psi^{b}$ is the restriction of $\Psi$ to $\mathrm{D}^{b}(\mathcal{V})$ and $\Psi^{0}: \mathcal{V} \longrightarrow \Psi(\mathcal{V})=\mathcal{H}_{V}$ is the naturally induced exact functor. Hence, real ${ }_{V}^{X}$ is an extendable equivalence.

(ii) $\Longrightarrow$ (i): First, from Theorem 2.8, there is a recollement of hearts of the form

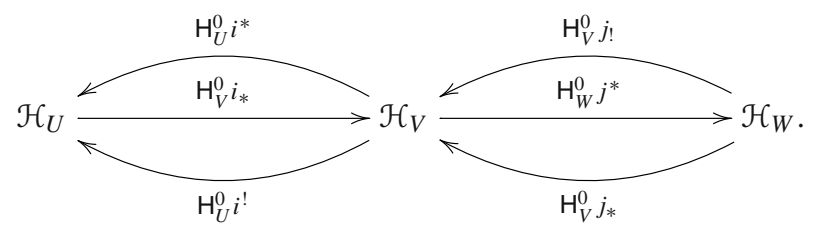

Note that there is a slight abuse of notation here: each of the functors in the recollement is in fact a triple composition-we are omitting the embedding of each heart in the corresponding triangulated category. Set $I_{*}:=\mathrm{H}_{V}^{0} i_{*}$ and $J^{*}:=\mathrm{H}_{W}^{0} j^{*}$ and keep the same notations for the corresponding derived functors.

Consider the f-category $(X, \theta)$ over $\mathrm{D}(\mathscr{A})$ and the realisation functor real ${ }_{V}^{X}$ which, by assumption, is an extendable equivalence. Let $(y, \xi)$ and $(\mathcal{Z}, \eta)$ be the induced f-categories over $\mathrm{D}(\mathscr{B})$ and $\mathrm{D}(\mathscr{C})$, respectively, so that $i_{*}$ and $j^{*}$ admit f-liftings (Proposition 3.8 and Corollary 3.9). Since $i_{*}$ and $j^{*}$ are t-exact functors for the fixed t-structures (Theorem 2.8), it follows from Theorem 3.13 that we have commutative diagrams
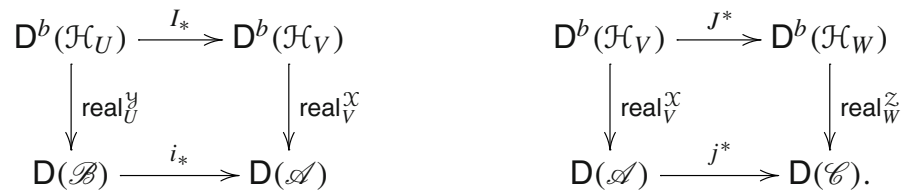
From the left diagram we show that the functor $I_{*}$ is a homological embedding. Let $X$ and $Y$ be objects in $\mathcal{H}_{U}$. Since real $\left.\right|_{U} ^{y}$ is fully faithful (see Proposition 5.1) and acts as the identity on $X$ and $Y[n]$ for any $n$, it follows that $\operatorname{Ext}_{\mathcal{H}_{U}}^{n}(X, Y) \cong$ $\operatorname{Hom}_{\mathrm{D}\left(\mathcal{H}_{U}\right)}(X, Y[n]) \cong \operatorname{Hom}_{\mathrm{D}(\mathscr{B})}(X, Y[n])$. Now, since $i_{*}$ is fully faithful, we get that $\operatorname{Ext}_{\mathcal{H}_{U}}^{n}(X, Y) \cong \operatorname{Hom}_{\mathrm{D}(\mathscr{A})}\left(i_{*} X, i_{*} Y[n]\right)$. On the other hand, since real $\left.\right|_{V} ^{X}$ is fully faithful (again by Proposition 5.1) and acts as the identity on $\mathrm{H}_{V}^{0} i_{*} X=i_{*} X$ and $\left(\mathrm{H}_{V}^{0} i_{*} Y\right)[n]=i_{*} Y[n]$, it follows that

$$
\operatorname{Ext}_{\mathcal{H}_{V}}^{n}\left(\mathrm{H}_{V}^{0} i_{*} X, \mathrm{H}_{V}^{0} i_{*} Y\right) \cong \operatorname{Hom}_{\mathrm{D}\left(\mathcal{H}_{V}\right)}\left(i_{*} X, i_{*} Y[n]\right) \cong \operatorname{Hom}_{\mathrm{D}(\mathscr{A})}\left(i_{*} X, i_{*} Y[n]\right),
$$

thus showing that $I_{*}$ is a homological embedding.

By assumption the functor real $\left.\right|_{V} ^{X}$ is extendable, that is, there is a restrictable equivalence, denoted by Real $\left.\right|_{V} ^{x}$, between $\mathrm{D}\left(\mathcal{H}_{V}\right)$ and $\mathrm{D}(\mathscr{A})$ that restricts to real $\left.\right|_{V} ^{x}$. In particular, $\mathrm{D}\left(\mathcal{H}_{V}\right)$ is TR5. Since all the other completeness properties required in Theorem 6.10 are also satisfied by assumption, the proof of that same theorem yields an exact sequence of triangulated categories:

$$
0 \longrightarrow \mathrm{D}\left(\mathcal{H}_{U}\right) \stackrel{I_{*}}{\longrightarrow} \mathrm{D}\left(\mathcal{H}_{V}\right) \stackrel{J^{*}}{\longrightarrow} \mathrm{D}\left(\mathcal{H}_{W}\right) \longrightarrow 0 .
$$

Consider now the composition $F:=j^{*} \operatorname{Real}_{V}^{x} I_{*}: \mathrm{D}\left(\mathcal{H}_{U}\right) \longrightarrow \mathrm{D}(\mathscr{C})$. Note that by the commutativity of (6.5), the image of $\mathrm{D}^{b}\left(\mathcal{H}_{U}\right)$ under $F$ is zero. Since $F$ is, by construction, texact with respect to the standard t-structure in $\mathrm{D}\left(\mathcal{H}_{U}\right)$ and the tilting t-structure generated by $W$ in $\mathrm{D}(\mathscr{C})$, we have $F\left(\mathrm{H}_{0}^{i}(X)\right)=\mathrm{H}_{W}^{i}(F(X))$ for any object $X$ in $\mathrm{D}\left(\mathcal{H}_{U}\right)$. But $\mathrm{H}_{0}^{i}(X)$ lies in $\mathrm{D}^{b}\left(\mathcal{H}_{U}\right)$ and, hence, we conclude that $\mathrm{H}_{W}^{i}(F(X))=0$ for all $i \in \mathbb{Z}$. Since tilting t-structures are nondegenerate (see Remark 4.4) it follows that $F \cong 0$. Hence the functor Real ${ }_{V}^{X}$ induces a functor $\Phi: \mathrm{D}\left(\mathcal{H}_{U}\right) \longrightarrow \mathrm{D}(\mathscr{A})$ such that $i_{*} \Phi=$ Real $_{V}^{X} I_{*}$. As a consequence, Real $\left.\right|_{V} ^{X}$ also induces a functor $\Theta: \mathrm{D}\left(\mathcal{H}_{W}\right) \longrightarrow \mathrm{D}(\mathscr{C})$ such that $\Theta J^{*}=j^{*} \operatorname{Real}_{V}^{X}$.

It remains to show that $\Phi$ (and, thus, $\Theta$ ) are (restrictable) triangle equivalences. Let $\Phi^{b}$ denote the restriction of $\Phi$ to $\mathrm{D}^{b}\left(\mathcal{H}_{U}\right)$. Since, by diagram (6.5), in the bounded setting we have $i_{*} \Phi^{b} \cong$ real $I_{V}^{x} I_{*} \cong i_{*}$ real $\left.\right|_{U} ^{y}$, it follows from the fully faithfulness of $i_{*}$ that $\Phi^{b} \cong$ real $\left.\right|_{U} ^{y}$. Therefore, since $U$ is a bounded tilting object, we get from Corollary 5.2 that the essential image of $\Phi^{b}$ is $\mathrm{D}^{b}(\mathscr{B})$. By the relation $i_{*} \Phi=$ Real ${ }_{V}^{x} I_{*}$ we get that $\Phi$ is fully faithful and therefore we can consider the essential image $\operatorname{Im} \Phi$ as a full subcategory of $\mathrm{D}(\mathscr{B})$. Then, since by assumption $\mathrm{D}\left(\mathcal{H}_{U}\right)$ is TR5, it follows that $\operatorname{lm} \Phi$ is a localising subcategory of $\mathrm{D}(\mathscr{B})$. Moreover, Im $\Phi$ contains $\mathrm{D}^{b}(\mathscr{B})$ and thus, from Lemma 6.6(i), since $\mathscr{B}$ is Grothendieck, it follows that $\operatorname{Im} \Phi=\mathrm{D}(\mathscr{B})$. We infer that the functor $\Phi$ is an equivalence, as wanted. Note that, similarly to the arguments above, it can also be seen that $\Theta^{b} \cong$ real $\left.\right|_{W} ^{z}$.

Finally, observe that $\Phi$ and $\operatorname{Real}_{V}^{\chi}$ preserve products and coproducts since they are equivalences. Since also $i_{*}: \mathrm{D}(\mathscr{B}) \longrightarrow \mathrm{D}(\mathscr{A})$ preserves products and coproducts, it follows that so does $I_{*}: \mathrm{D}\left(\mathcal{H}_{U}\right) \longrightarrow \mathrm{D}\left(\mathcal{H}_{V}\right)$. Hence, by Theorem 6.10, the functor $I_{*}$ induces indeed a recollement of unbounded derived categories.

The following result (Theorem $\mathrm{C}$ in the introduction) is a consequence of the above theorem and it provides necessary and sufficient conditions for a recollement of derived module categories to be equivalent to a stratifying recollement (recall Definition 2.6). In this case almost all technical assumptions of Theorem 6.13 are automatically satisfied. In order to guarantee the extendability of a realisation functor, we assume the ring in the middle of the recollement to be a projective $\mathbb{K}$-algebra over a commutative ring $\mathbb{K}$. The statement reads then as follows. 
Corollary 6.14 Let $A, B$ and $C$ be rings. Assume that $A$ is a projective $\mathbb{K}$-algebra over a commutative ring $\mathbb{K}$. Suppose there is a recollement $\mathcal{R}$ of the form

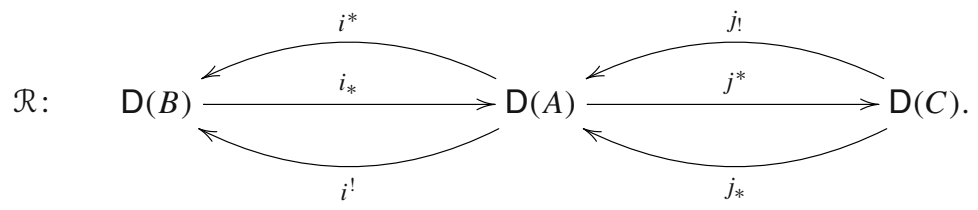

Then the following are equivalent.

(i) The recollement $\mathcal{R}$ is equivalent to a stratifying recollement of a projective $\mathbb{K}$-algebra $S$.

(ii) There are compact tilting objects $V$ in $\mathrm{D}(A), U$ in $\mathrm{D}(B)$ and $W$ in $\mathrm{D}(C)$ such that the associated tilting $t$-structures in $\mathrm{D}(B)$ and $\mathrm{D}(C)$ glue along $\mathcal{R}$ to the associated tilting $t$-structure in $\mathrm{D}(A)$ and such that $\mathrm{End}_{\mathrm{D}(A)}(V)$ is a projective $\mathbb{K}$-algebra.

Proof We use the fact that a recollement of module categories is equivalent to a recollement induced by an idempotent element ([70, Theorem 5.3]). This corollary then becomes a direct application of Theorem 6.13, provided we show that in this setting the additional technical assumptions of the theorem are automatically satisfied. First note that both derived module categories and their duals satisfy Brown representability (they are left-complete derived categories of a Grothendieck category, see Example 6.4(v) and Theorem 6.5). Note, furthermore, that every equivalence between derived module categories is restrictable (this follows from Example 3.16(i)). Since we assume that the algebra $A$ is projective over $\mathbb{K}$, it also follows that the realisation functor of the compact tilting object $V$ with respect to $\operatorname{DF}(A)$ is an extendable equivalence since it is an equivalence of standard type (see Theorem 5.13). Finally, observe that the compactness of the tilting objects is used to produce hearts which are module categories (see Corollary 4.7).

In the next result we show, using Lemma 6.12(ii), that we can be more specific about the shape of equivalences between two stratifying recollements (compare with [57, Theorem $3.5])$.

Corollary 6.15 Let $\mathbb{K}$ be a commutative ring, $A$ and $B$ projective $\mathbb{K}$-algebras and $e$ and $u$ idempotents in $A$ and $B$ respectively such that $A / A e A, e A e, B / B u B$ and $u B u$ are also projective $\mathbb{K}$-algebras. Suppose that $f: A \longrightarrow A / A e A$ and $g: B \longrightarrow B / B u B$ are homological ring epimorphisms, and denote by $\mathcal{R}_{f}$ and $\mathcal{R}_{g}$ the induced recollements of unbounded derived module categories. The following statements are equivalent.

(i) There is an equivalence of recollements from $\mathcal{R}_{f}$ to $\mathcal{R}_{g}$ with all equivalences being extensions to unbounded derived categories of equivalences of standard type between bounded derived categories.

(ii) There are compact tilting objects $V$ in $\mathrm{D}(B), U$ in $\mathrm{D}(B / B u B)$ and $W$ in $\mathrm{D}(u B u)$ such that

$$
\operatorname{End}_{\mathrm{D}(B)}(V) \cong A, \operatorname{End}_{\mathrm{D}(B / B u B)}(U) \cong A / A e A, \operatorname{End}_{\mathrm{D}(u B u)}(W) \cong e A e
$$

and the associated tilting t-structures in $\mathrm{D}(B / B u B)$ and $\mathrm{D}(u B u)$ glue along $\mathcal{R}_{g}$ to the associated tilting t-structure in $\mathrm{D}(A)$.

Proof Following the proof of the Theorem 6.13, we see that the choice of the f-categories for the realisation functors that yield the equivalence of recollements is the one provided by 
Proposition 3.8. We start with the recollement induced by $g$ and, thus, with a recollement of abelian categories coming from a homological embedding. In this setting, Lemma 6.12(ii) shows that if we chose the f-category over $\mathrm{D}(B)$ to be the filtered derived category $\operatorname{DF}(B)$, then the induced f-categories over $\mathrm{D}(B / B u B)$ and $\mathrm{D}(u B u)$ are, respectively, the filtered derived categories $\operatorname{DF}(B / B u B)$ and $\operatorname{DF}(u B u)$. The result then follows from the fact that the equivalences built in the proof of Theorem 6.13 are extensions of realisation functors of compact tilting objects with respect to filtered derived categories. These realisation functors are, therefore, derived equivalences of standard type by Theorem 5.13, finishing the proof.

At this point we cannot prove with our techniques that the simple formulation of Corollary 6.14 holds for arbitrary rings (compare with [7]). The main obstacle there is the existence of an extension of the realisation functor. In Corollary 6.15, the problem occurring is that we do not know whether an extension of an equivalence of bounded derived categories to an equivalence of unbounded derived categories is unique. Although we know that equivalences of standard type are extendable, we do not know whether the extensions obtained in the proof of Theorem 6.13 coincide with the expected derived tensor product.

If, however, we turn our attention to recollements of bounded derived categories, we can formulate an analogue of Corollary 6.14 even with more general assumptions.

Corollary 6.16 Let $\mathscr{A}, \mathscr{B}$ and $\mathscr{C}$ be abelian categories with a projective generator and such that their unbounded derived categories are TR5 and TR5*. Suppose that there is a recollement of bounded derived categories:

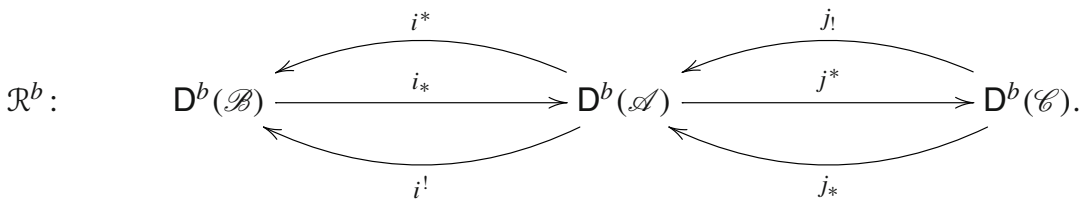

Assume that the global dimension of $\mathscr{A}$ or $\mathscr{C}$ is finite. The following statements are equivalent.

(i) The recollement $\mathcal{R}^{b}$ is equivalent to the restriction of a stratifying recollement to bounded derived categories.

(ii) There are compact tilting objects $V$ in $\mathrm{D}(\mathscr{A}), U$ in $\mathrm{D}(\mathscr{B})$ and $W$ in $\mathrm{D}(\mathscr{C})$ such that the associated tilting t-structures in $\mathrm{D}(\mathscr{B})$ and $\mathrm{D}(\mathscr{C})$ glue along $\mathcal{R}^{b}$ to the associated tilting $t$-structure in $\mathrm{D}(A)$.

Proof Note that (i) $\Longrightarrow$ (ii) follows as in the proof of Theorem 6.13. Conversely, following the arguments in the proof of Theorem 6.13, it easily follows that the induced fully faithful functor $\mathcal{H}_{U} \longrightarrow \mathcal{H}_{V}$ is homological. Since all the hearts are module categories (the tilting objects are compact, see Corollary 4.7), it follows from [70] that the recollement of hearts is equivalent to one induced by a homological ring epimorphism $f: S \longrightarrow S / S e S$, where $S$ is Morita equivalent to $\mathrm{End}_{\mathrm{D}(\mathscr{A})}(V)$. Since $\mathscr{A}$ or $\mathscr{C}$ have finite global dimension, then so does $S$ or $e S e$ (see Proposition 5.8). In any of these two cases, it follows from [69, Theorem 7.2] that $f: S \longrightarrow S / \mathrm{SeS}$ induces a recollement of bounded derived categories. It remains to show that this recollement is equivalent to $\mathcal{R}^{b}$. However, this follows from the same arguments used in the proof of Theorem 6.13, omitting the issues related to the extendability of the realisation functors.

We finish with an application of the above results to recollements of derived module categories of finite dimensional hereditary $\mathbb{K}$-algebras, over a field $\mathbb{K}$. 
Theorem 6.17 Let $A$ be a finite dimensional hereditary $\mathbb{K}$-algebra over a field $\mathbb{K}$. Then any recollement of $\mathrm{D}(A)$ by derived module categories is equivalent to a stratifying one.

Proof Let $\mathcal{R}$ be a recollement of $\mathrm{D}(A)$ of the form

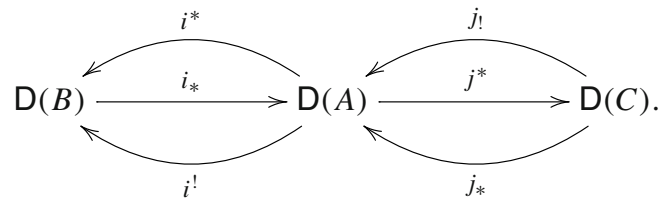

It follows from the assignments in [55, Theorem 8.1] (see also [5, Corollary 3.3]) for hereditary rings that $\mathcal{R}$ is equivalent to a recollement induced by a homological ring epimorphism. Moreover, this equivalence changes only the functors between $\mathrm{D}(B)$ and $\mathrm{D}(A)$, leaving the functors between $\mathrm{D}(A)$ and $\mathrm{D}(C)$ unchanged. Thus, without loss of generality, we assume $i_{*}=f_{*}$ for some homological ring epimorphism $f: A \longrightarrow B$.

Now, since $A$ is a finite dimensional algebra of finite global dimension, the recollement $\mathcal{R}$ fits in an infinite ladder (see [6, Proposition 3.7]). In particular, the functors $i^{*}$ and $j$ ! (respectively, $i^{!}$and $j_{*}$ ) admit left (respectively, right) adjoints and there is a recollement

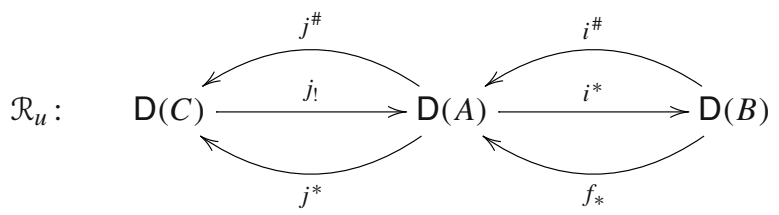

Applying once again the result quoted in the first paragraph, there is a recollement of $\mathrm{D}(A)$ equivalent to $\mathcal{R}_{u}$, which is induced by a homological ring epimorphism (and the functors between $\mathrm{D}(A)$ and $\mathrm{D}(B)$ remain unchanged). Thus, without loss of generality, once again we assume that $j !=g_{*}$ for some homological ring epimorphism $g: A \longrightarrow C$.

Denote by $\mathbb{T}$ the glueing of the standard t-structures in $\mathrm{D}(C)$ and $\mathrm{D}(B)$ along $\mathcal{R}$. We check that $\mathbb{T}$ is a tilting t-structure in $\mathrm{D}(A)$. The standard t-structures in $\mathrm{D}(C)$ and $\mathrm{D}(B)$ admit left adjacent co-t-structures (see [21] for the definition). These co-t-structures, when glued along $\mathcal{R}_{u}$, yield a left adjacent co-t-structure to $\mathbb{T}$ (see [56, Remark 2.6]). Note that, since $A$ has finite global dimension, then so do $B$ and $C$ ([6, Proposition 2.14]) and, hence, $\mathcal{R}$ restricts to a recollement of $\mathrm{D}^{b}(A)$ ([6, Corollary 4.10]). Since the standard t-structures are bounded, then $\mathbb{T}$ restricts to a bounded t-structure in $\mathrm{D}^{b}(A)$ and therefore Lemma 4.14(iii) implies that $\mathbb{T}$ is an intermediate t-structure. From [8, Theorem 4.6], any intermediate t-structure that admits a left adjacent co-t-structure is a (bounded) silting t-structure and, thus, there is a bounded silting object $T$ such that $\mathbb{T}=\mathbb{T}_{T}$.

Since $A$ is a finite dimensional $\mathbb{K}$-algebra, then so are $B$ and $C$ ([6, Lemma 2.10(b)]). It follows that the recollements $\mathcal{R}$ and $\mathcal{R}_{u}$ also restrict to the level of bounded derived categories of finitely generated modules (see, for example, [6, Theorem 4.4]). From [56], $T$ is then compact and it can be computed explicitly. More precisely, following the terminology of [56], $T$ is the glued silting object of $B$ and $C$ along $\mathcal{R}_{u}$. From [56, Theorem 4.5], $T$ is tilting if and only if the following conditions hold:

(i) $\operatorname{Hom}_{\mathrm{D}(C)}\left(C, j^{\#} f_{*} B[k]\right)=0$, for all $k<-1$;

(ii) $\operatorname{Hom}_{\mathrm{D}(C)}\left(j^{\#} f_{*} B, C[k]\right)=0$, for all $k<0$;

(iii) $\operatorname{Hom}_{\mathrm{D}(C)}\left(j^{\#} f_{*} B, j^{\#} f_{*} B[k]\right)=0$, for all $k<-1$. 
We start by analysing the object $j^{\#} f_{*} B$. Since $j^{\#}$ is the left adjoint of $j_{!}=g_{*}$, we conclude that $j^{\#}$ is naturally equivalent to $-\otimes_{A}^{\mathbb{L}} C$. Since $C$ has projective dimension at most 1 as an $A$-module and $f_{*} B$ is an $A$-module, it follows that $j^{\#} f_{*} B$ is a 2-term complex in $\mathrm{D}(C)$ with cohomologies concentrated in degrees 0 and -1 . From this property, it is then obvious that conditions (i), (ii) and (iii) are satisfied and, thus, the glued object from $C$ and $B$ along $\mathcal{R}_{u}$ is tilting. The result then follows from Corollary 6.14 .

Remark 6.18 (i) Starting with an arbitrary recollement $\mathcal{R}$ of $\mathrm{D}(A)$ by derived module categories as in Theorem 6.17, in general one needs to change all three terms by nontrivial derived equivalences in order to obtain a stratifying recollement. In the above proof, we change the left hand side to a derived equivalent ring that induces $\mathcal{R}$ via a homological ring epimorphism of $A$. Then we change the right hand side (i.e. the left hand side of $\mathcal{R}_{u}$ ) in the same way. Finally, we change the middle term by considering the derived equivalence given by the tilting object obtained by glueing.

(ii) In the proof of the above theorem we use the fact that we can restrict the recollement $\mathcal{R}$ and $\mathcal{R}_{u}$ to recollements of $\mathrm{D}^{b}(A)$ and of $\mathrm{D}^{b}(\bmod -A)$. It is then not difficult to see that the proof of the above theorem yields that, for $A$ a finite dimensional hereditary $\mathbb{K}$ algebra, any recollement of $\mathrm{D}^{b}(A)$ (or of $\mathrm{D}^{b}(\bmod -A)$ ) by bounded derived categories $\mathrm{D}^{b}(B)$ and $\mathrm{D}^{b}(C)$ (respectively, by $\mathrm{D}^{b}(\bmod -B)$ and $\left.\mathrm{D}^{b}(\bmod -C)\right)$ is the restriction to $\mathrm{D}^{b}(S)$ (respectively $\mathrm{D}^{b}(\bmod -S)$ ) of a stratifying recollement of a finite dimensional $\mathbb{K}$-algebra $S$ derived equivalent to $A$.

Acknowledgements The authors would like to thank Lidia Angeleri Hügel and Steffen Koenig for their support in this project and their detailed comments on a preliminary version of this paper; Olaf Schnürer for his help in clarifying the proof of Theorem 3.13 and Greg Stevenson for suggesting the use of a dévissage argument for the proof of Theorem 6.9; Silvana Bazzoni, Henning Krause, Pedro Nicolás and Manuel Saorin for many discussions, questions and comments regarding this project; Changchang Xi and his research group at the Capital Normal University for many questions and comments on this work during a visit of the first named author; the University of Bielefeld, the University of Stuttgart, the University of Verona and the Norwegian University of Science and Technology for hosting the authors in several occasions of their continued collaboration.

Open Access This article is distributed under the terms of the Creative Commons Attribution 4.0 International License (http://creativecommons.org/licenses/by/4.0/), which permits unrestricted use, distribution, and reproduction in any medium, provided you give appropriate credit to the original author(s) and the source, provide a link to the Creative Commons license, and indicate if changes were made.

\section{Appendix A: On Beilinson's realization functor (by Ester Cabezuelo Fernández and Olaf M. Schnürer ${ }^{1}$ )}

Given a t-structure with heart $\odot$ on a triangulated category $\mathcal{T}$, a classical problem is the construction of a t-exact, and hence in particular triangulated, functor $\mathrm{D}^{b}(\mathcal{Q}) \rightarrow \mathcal{T}$ extending the inclusion $\odot \rightarrow \mathcal{T}$ (up to isomorphism). Such a functor is called a realization functor.

Beilinson claims in [15, Appendix] that a realization functor exists if $\mathcal{T}$ admits a filtered triangulated category. Following Beilinson's hints, it is possible to construct a functor $\mathrm{D}^{b}(\mathrm{O}) \rightarrow \mathcal{T}$ which is (isomorphic to) the identity on $\odot$. This functor is compatible with the shift functor on the source category and the suspension functor on its target. However, we could not prove that it is triangulated. To us, it seems unavoidable to impose an additional

1 Mathematisches Institut, Universität Bonn, Endenicher Allee 60, 53115 Bonn, Germany. E-mail addresses: ester.cabezuelo@gmail.com,olaf.schnuerer@math.uni-bonn.de. 
axiom on the filtered triangulated category to ensure this. This axiom says that a certain family of morphisms of triangles can be completed to a $3 \times 3$-diagram of triangles (see Appendix A.1).

Interestingly, the same additional axiom was used in the context of weight structures (or co-t-structures) in [74] to ensure the existence of a strong weight complex functor under suitable assumptions. Motivated by these two instances and our failed efforts to do without this axiom, we strongly believe that this axiom should be added to the definition of a filtered triangulated category.

In this appendix we explain how the additional axiom is used in order to show that the realization functor is triangulated, under the assumption that it is already constructed as a functor. Full details will appear in the master thesis [22] and presumably in a subsequent publication.

\section{A.1 The additional axiom}

We restate the additional axiom (fcat7) from [74, 7.2]. We use notation and basic facts about filtered triangulated categories from this article.

Let $(\widetilde{\mathcal{T}}, \widetilde{\mathcal{T}}(\leq 0), \widetilde{\mathcal{T}}(\geq 0), s, \alpha)$ be a filtered triangulated category. The additional axiom we need is the following condition which expresses a certain compatibility between the $\sigma$ truncation triangles and the morphism $\alpha:$ id $_{\tilde{\mathcal{T}}} \rightarrow s$ of triangulated functors.

(fcat7) For any morphism $f: X \rightarrow Y$ in $\widetilde{\mathcal{T}}$ the morphism

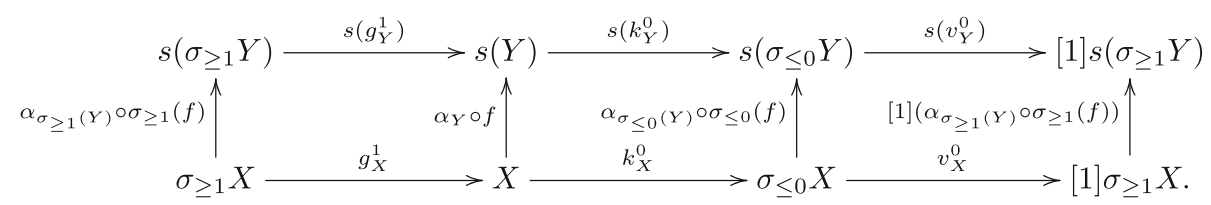

of triangles can be extended to a $3 \times 3$-diagram of triangles. In other words, this morphism of triangles is middling-good in Neeman's terminology [61, Def. 2.4].

\section{A.2. The realization functor is triangulated}

Let $(\widetilde{\mathcal{T}}, \widetilde{\mathcal{T}}(\leq 0), \tilde{\mathcal{T}}(\geq 0), s, \alpha)$ be a filtered triangulated category over a triangulated category $\mathcal{T}$, i. e. we are given an equivalence $\mathcal{T} \stackrel{\sim}{\rightarrow} \widetilde{\mathcal{T}}([0])=\widetilde{\mathcal{T}}(\leq 0) \cap \widetilde{\mathcal{T}}(\geq 0)$ of triangulated categories.

Recall from [74, Prop. 6.9] the functor

$$
c:\left(\widetilde{\mathcal{T}},[1] s^{-1}\right) \rightarrow\left(\mathrm{C}^{b}(\mathcal{T}), \Sigma\right)
$$

of additive categories with translation (we use the notation [1] for suspension in $\widetilde{T}$ and $\Sigma$ for the shift of a complex): on objects $X \in \tilde{\mathcal{T}}$ there is a given isomorphism

$$
c\left([1] s^{-1}(X)\right) \cong \Sigma c(X) .
$$

Now assume that $\mathcal{T}$ is endowed with a t-structure $(\mathcal{T} \leq 0, \mathcal{T} \geq 0$ ). Then there is a compatible t-structure $(\widetilde{\mathcal{T}} \leq 0, \widetilde{\mathcal{T}} \geq 0$ ) on $\widetilde{\mathcal{T}}$, as claimed in [15]. It is uniquely characterized by the fact that the given equivalence $\mathcal{T} \stackrel{\sim}{\rightarrow} \widetilde{\mathcal{T}}([0])$ is t-exact and that $s(\tilde{\mathcal{T}} \leq 0)=\tilde{\mathcal{T}} \leq-1$. This implies that $s(\tilde{\mathcal{T}} \geq 0)=\widetilde{\mathcal{T}} \geq-1$. 
If we denote the hearts of these t-structures by $\odot$ and $\widetilde{\odot}$, respectively, the functor (A.1) restricts to an equivalence

$$
c: \widetilde{\sim} \stackrel{\sim}{\rightarrow} \mathrm{C}^{b}(\odot)
$$

of abelian categories, as claimed in [15]. Note that the heart $\widetilde{\nabla}$ is stable under [1] $s^{-1}$. Moreover, it is stable under the functors $\sigma_{\geq n}$ and $\sigma_{\leq n}$, and these functors (together with the transformations $\sigma_{\geq n} \rightarrow$ id and id $\rightarrow \sigma_{\leq n}$ ) correspond to the brutal truncation functors on $\mathrm{C}^{b}(\mathrm{Q})$

Consider the diagram

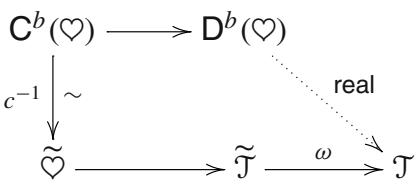

of categories where $c^{-1}$ is a quasi-inverse to the equivalence $c$, the upper horizontal arrow is the localization with respect to all quasi-isomorphisms, $\mathrm{D}^{b}(\mathrm{Q})$ is the bounded derived category of $\odot$, and the arrows in the lower row are the inclusion functor and the triangulated functor "forget the filtration" $\omega$ (see [74, Prop. 6.6]). All functors are functors of categories with translation. It can be shown that the dotted arrow exists uniquely as a functor of categories with translation such that the diagram is commutative, as claimed in [15]. This functor is the realization functor. Its restriction to $\odot$ is isomorphic to the inclusion $\varnothing \rightarrow \mathcal{T}$.

Remark $A .1$ If we assume that $\mathcal{T}=\widetilde{\mathcal{T}}([0])$ and choose $\omega$ and $c^{-1}$ wisely, then the restriction of the realization functor to $\odot$ is the inclusion of $\odot$ into $\mathcal{T}$.

If $f^{\prime}: X^{\prime} \rightarrow Y^{\prime}$ is a morphism in $\mathrm{C}^{b}(\odot)$, let

$$
X^{\prime} \stackrel{f^{\prime}}{\rightarrow} Y^{\prime} \stackrel{\left(\begin{array}{l}
1 \\
0
\end{array}\right)}{\longrightarrow} \operatorname{cone}\left(f^{\prime}\right) \stackrel{(01)}{\longrightarrow} \Sigma X^{\prime}
$$

be the usual mapping cone sequence where the cone cone $\left(f^{\prime}\right)$ is given by $\operatorname{cone}\left(f^{\prime}\right)^{n}=$ $Y^{\prime n} \oplus X^{\prime n+1}$ with differential $\left[\begin{array}{cc}d & f \\ 0 & -d\end{array}\right]$.

Our main result, Theorem A.3, will be an easy consequence of the following technical proposition.

Proposition A.2 Keep the above assumptions and assume that $\widetilde{\mathcal{T}}$ satisfies axiom (fcat7). Let $f: X \rightarrow Y$ be a morphism in $\widetilde{\odot}$. Complete the morphism $f \circ \alpha=f \circ \alpha_{s^{-1} X}: s^{-1} X \rightarrow Y$ to a triangle

$$
s^{-1} X \stackrel{f \circ \alpha}{\rightarrow} Y \stackrel{g}{\rightarrow} Z \stackrel{h}{\rightarrow}[1] s^{-1} X
$$

in $\widetilde{\mathcal{T}}$. Then there is an isomorphism $m$ such that the diagram

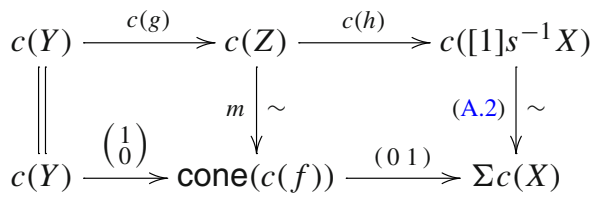

in $\mathrm{C}^{b}(\mathrm{O})$ is commutative. 
Before giving the proof, we remind the reader of the definition of the functor (A.1) on the subcategory $\widetilde{\mathcal{T}}([-1,0])$. Given an object $A \in \widetilde{\mathcal{T}}([-1,0])$, consider the diagram

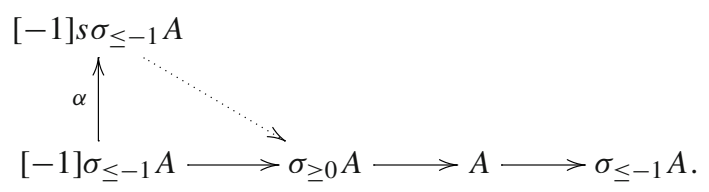

Its lower row is obtained by rotation from the obvious $\sigma$-truncation triangle of $A$. The dotted arrow exists uniquely such that the diagram is commutative, and $c(A)$ is the dotted arrow viewed as a complex concentrated in degrees $-1,0$. This construction is clearly functorial.

Proof Let $a \leq b$ be integers such that $X$ and $Y$ are objects of $\widetilde{\mathcal{T}}([a, b])$. We prove the proposition by induction over $b-a$.

Base case $a=b$. Without loss of generality we can assume that $a=b=0$.

We claim that the conclusion of the proposition is even true for all morphisms $f: X \rightarrow Y$ in $\widetilde{\mathcal{T}}([0])$. Note that $[1] s^{-1} X \in \widetilde{\mathcal{T}}([-1])$. Hence $Z \in \widetilde{\mathcal{T}}([-1,0])$ and the triangle (A.4) identifies in a unique way with the triangle in (A.5) for $A=Z$. Hence $f$ identifies with the dotted arrow and $c(Z)$ with the complex $(\cdots \rightarrow 0 \rightarrow X \stackrel{f}{\rightarrow} Y \rightarrow 0 \rightarrow \cdots)$.

Similarly, the triangle $0 \rightarrow Y \stackrel{\text { id }}{\rightarrow} Y \rightarrow 0$ identifies with the triangle (A.5) for $A=Y$, and hence $c(Y)$ with $Y$ viewed as a complex in degree zero; the triangle $s^{-1} X \rightarrow 0 \rightarrow$ $[1] s^{-1} X \stackrel{\text { id }}{\rightarrow}[1] s^{-1} X$ identifies with the triangle (A.5) for $A=[1] s^{-1} X$, and hence $c\left([1] s^{-1} X\right)$ with $X$ viewed as a complex in degree -1 , and this identification coincides with (A.2).

It is easy to see that the morphisms $c(Y) \stackrel{c(g)}{\longrightarrow} c(Z) \stackrel{c(h)}{\longrightarrow} c\left([1] s^{-1} X\right)$ correspond under these identifications to the obvious morphisms

$$
(0 \rightarrow Y) \rightarrow(X \stackrel{f}{\rightarrow} Y) \rightarrow(X \rightarrow 0)
$$

of complexes concentrated in degrees $-1,0$. This proves the base case of our induction.

Induction step. Assume now that $f: X \rightarrow Y$ is a morphism in $\widetilde{\mathcal{T}}([a, b]) \cap \widetilde{\widetilde{O}}$. Without loss of generality we can assume that $a \leq 1<b$. By induction, we already know the conclusion of the proposition for the morphisms $\sigma_{\geq 2} f$ and $\sigma_{\leq 1} f$.

Axiom (fcat7) applied to the morphism $s^{-1} f: s^{-1} X \rightarrow s^{-1} Y$ shows that the following morphism of triangles is middling-good.

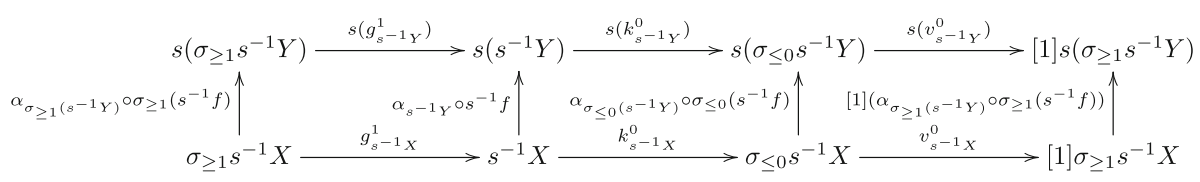

We claim that this morphism of triangles is isomorphic to the following morphism of triangles.

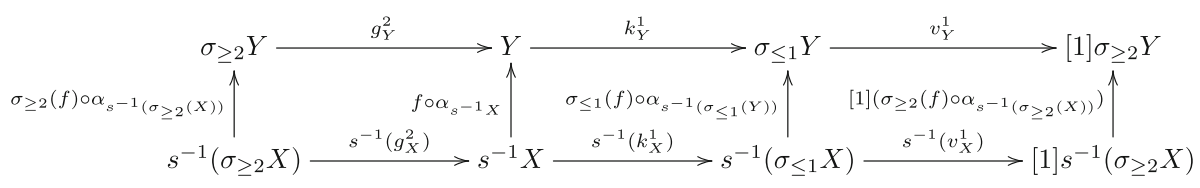


This is straightforward to prove: The two lower triangles in (A.6) and (A.7) are uniquely isomorphic by a morphism of triangles whose middle component is the identity. This isomorphism of triangles is functorial in $X$. Hence $f: X \rightarrow Y$ yields a morphism between these isomorphisms of triangles; in other words, we obtain a commutative square whose vertices are triangles and whose arrows are morphisms of triangles, two of them being isomorphisms. Applying the morphism $\alpha$ of triangulated functors to this square yields our claim.

Hence also the morphism (A.7) of triangles is middling-good, say it fits into the following $3 \times 3$-diagram whose right-most column we do not draw.

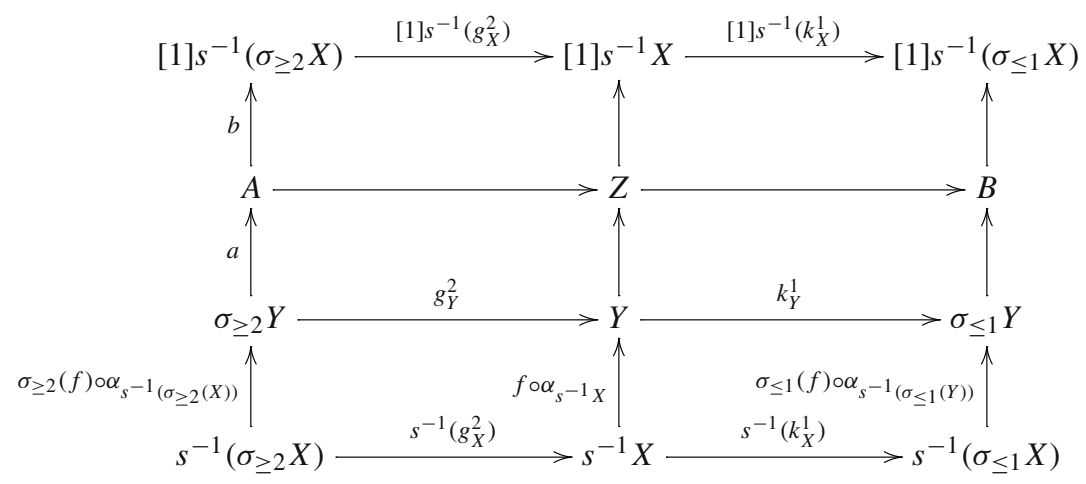

In the following we will only need that all rows can be extended to triangles, that all columns are triangles, and that the diagram is commutative.

Now we use the assumption that $X$ and $Y$ are objects of the heart $\widetilde{\nabla}$. If we ignore the bottom row of this diagram, we obtain a $3 \times 3$-diagram of nine objects that are easily seen to be objects of the heart $\widetilde{\nabla}$. More precisly, we obtain a commutative $3 \times 3$-diagram in the abelian category $\widetilde{\nabla}$ all of whose rows and columns are short exact sequences since they come from triangles.

Using induction (and the isomorphism (A.2) and the fact that the functors $\sigma_{\geq n}, \sigma_{\leq n}$ correspond to the brutal truncations), the image of this diagram under the equivalence $c$ in (A.3) is isomorphic to the commutative diagram (A.9) below of short exact sequences in $\mathrm{C}^{b}(\mathrm{O})$ where we use the following notation: $f^{\prime}: X^{\prime} \rightarrow Y^{\prime}$ is the image of $f: X \rightarrow Y$ under the functor $c$, and $f_{\geq 2}^{\prime}: X_{\geq 2}^{\prime} \rightarrow Y_{\geq 2}^{\prime}$ and $f_{\leq 1}^{\prime}: X_{\leq 1}^{\prime} \rightarrow Y_{\leq 1}^{\prime}$ are the brutal truncations of $f^{\prime}$.

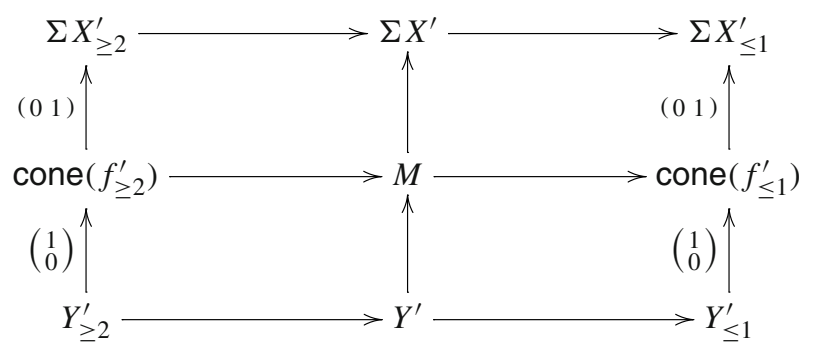

The horizontal arrows in top and bottom row are the usual morphisms from brutal truncation.

We need to see that there is an isomorphism $M \stackrel{\sim}{\rightarrow} \operatorname{cone}\left(f^{\prime}\right)$ such that the middle column of our diagram is identified with the obvious part of the usual mapping cone sequence of $f^{\prime}$. 
In degree one, diagram (A.9) looks as follows.

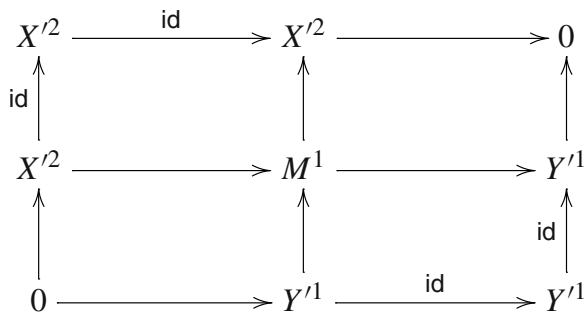

The epimorphism in the middle column of this diagram is split by the monomorphism in its middle row. We obtain an isomorphism $M^{1} \stackrel{\sim}{\rightarrow} Y^{\prime 1} \oplus X^{\prime 2}$ such that the four arrows ending and starting at $M^{1}$ correspond to the obvious inclusions and projections.

In degrees $n \neq 1$ it is even simpler to find similar isomorphisms $M^{n} \stackrel{\sim}{\rightarrow} Y^{\prime n} \oplus X^{\prime n}$ : the right column of diagram (A.9) is zero in degrees $n>1$, and its left column is zero in degrees $n<1$.

In this way we obtain an isomorphism $M \stackrel{\sim}{\rightarrow} Y^{\prime} \oplus \Sigma X^{\prime}$ of graded objects in $\odot$ such that all morphisms starting and ending in $M$ correspond to the obvious inclusions and projections. An easy computation shows that the differential of $M$ has the form $\left[\begin{array}{cc}d & f \\ 0 & -d\end{array}\right]$ modulo this isomorphism. This proves the proposition.

Theorem A.3 Keep the above assumptions and assume that $\widetilde{\mathcal{T}}$ satisfies axiom (fcat 7$)$. Then the realization functor real: $\mathrm{D}^{b}(\mathrm{Q}) \rightarrow \mathcal{T}$ is triangulated.

Proof Let $f: X \rightarrow Y$ be a morphism in $\widetilde{\mathcal{T}}$, and fit $f \circ \alpha$ into a triangle (A.4). Consider the sequence

$$
X \stackrel{f}{\rightarrow} Y \stackrel{g}{\rightarrow} Z \stackrel{h}{\rightarrow}[1] s^{-1} X
$$

in $\widetilde{\odot}$. Its image under $c$ is the first row in the following commutative diagram in $C^{b}(\odot)$ obtained from Proposition A.2.

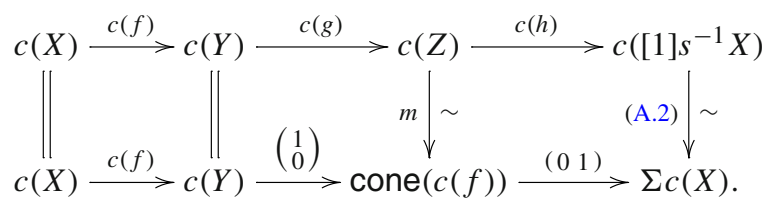

Since any triangle in $\mathrm{D}^{b}(\mathrm{O})$ is isomorphic to the image of mapping cone sequence in $\mathrm{C}^{b}(\mathrm{O})$, the equivalence (A.3) and our description of the realization functor real show that it is enough to see that the functor "forget the filtration" $\omega$ maps the sequence (A.10) in $\widetilde{\nabla}$ to a triangle in $\mathcal{T}$. But this is easy to see. First, the functor $\omega$ is triangulated and hence maps the triangle (A.4) to a triangle in $\mathcal{T}$. Second, $\omega(\alpha)$ is an isomorphism, by the very definition of $\omega$. This proves the theorem.

\section{References}

1. Aihara, T., Iyama, O.: Silting mutation in triangulated categories. J. Lond. Math. Soc. 85(3), 633-668 (2012) 
2. Alonso Tarrio, L., Jeremias López, A., Souto Salorio, M.J.: Construction of t-structures and equivalences of derived categories. Trans. Am. Math. Soc. 355, 2523-2543 (2003)

3. Alonso Tarrio, L., Jeremias López, A., Souto Salorio, M.J.: Localizations in categories of complexes and unbounded resolutions. Can. J. Math. 52, 225-247 (2000)

4. Angeleri Hügel, L., Coelho, F.U.: Infinitely generated tilting modules of finite projective dimension. Forum Math. 13, 239-250 (2001)

5. Angeleri Hügel, L., Koenig, S., Liu, Q.: On the uniqueness of stratifications of derived module categories. J. Algebra 359, 120-137 (2012)

6. Angeleri Hügel, L., Koenig, S., Liu, Q., Yang, D.: Ladders and simplicity of derived module categories. J. Algebra 472, 15-66 (2017)

7. Angeleri Hügel, L., Koenig, S., Liu, Q., Yang, D.: Recollements and stratifying ideals. J. Algebra 484, 47-65 (2017)

8. Angeleri Hügel, L., Marks, F., Vitória, J.: Silting modules. Int. Math. Res. Not. 2016(4), 1251-1284 (2016)

9. Angeleri Hügel, L., Saorin, M.: t-structures and cotilting modules over commutative noetherian rings. Math. Z. 277, 847-866 (2014)

10. Anno, R., Logvinenko, T.: On adjunctions for Fourier-Mukai transforms. Adv. Math. 231, 2069-2115 (2012)

11. Asadollahi, J., Hafezi, R., Vahed, R.: Bounded derived categories of infinite quivers: Grothendieck duality, reflection functor. Can. J. Math. 67, 28-54 (2015)

12. Bazzoni, S.: Equivalences induced by infinitely generated tilting modules. Proc. Am. Math. Soc. 138, 533-544 (2010)

13. Bazzoni, S.: The t-structure induced by an $n$-tilting module, preprint (2016). arXiv: 1604.00797

14. Bazzoni, S., Mantese, F., Tonolo, A.: Derived equivalence induced by infinitely generated $n$-tilting modules. Proc. Am. Math. Soc. 139, 4225-4234 (2011)

15. Beilinson, A.: On the derived category of perverse sheaves, In: K-theory, arithmetic and geometry (Moscow, 1984-1986), Lecture Notes in Math., 1289. Springer, Berlin (1987)

16. Beilinson, A., Bernstein, J., Deligne, P.: Faisceaux Pervers, (French) [Perverse sheaves], Analysis and topology on singular spaces, I (Luminy, 1981), pp. 5-171, Asterisque, 100. Soc. Math., France (1982)

17. Beligiannis, A., Reiten, I.: Homological and homotopical aspects of torsion theories, Mem. Am. Math. Soc. 188(883), viii+207 (2007)

18. Bökstedt, M., Neeman, A.: Homotopy limits in triangulated categories. Compos. Math. 86, 209-234 (1993)

19. Breaz, S., Pop, F.: Cosilting modules. Algebra Represent. Theory (2017). doi:10.1007/s10468-017-9688$\mathrm{X}$

20. Bridgeland, T.: t-structures on some local Calabi-Yau varities. J. Algebra 289, 453-483 (2005)

21. Bondarko, M.V.: Weight structures vs. t-structures; weight filtrations, spectral sequences, and complexes (for motives and in general). J K-Theory 6(3), 387-504 (2010)

22. Fernández, C.: Master thesis, Universität Bonn (2017) (in preparation)

23. Chen, H., Xi, C.C.: Good tilting modules and recollements of derived module categories. Proc. Lond. Math. Soc. 104(5), 959-996 (2012)

24. Chen, X.-W.: A note on standard equivalences. Bull. Lond. Math. Soc. 48(5), 797-801 (2016)

25. Chen, X.-W., Ye, Y.: The D-standard and K-standard categories, preprint (2016). arXiv:1612.06051

26. Cline, E., Parshall, B., Scott, L.: Algebraic stratification in representation categories. J. Algebra 117, 504-521 (1988)

27. Cline, E., Parshall, B., Scott, L.: Stratifying endomorphism algebras, Mem. Am. Math. Soc. 124(591), viii+119pp (1966)

28. Colpi, R.: Tilting in Grothendieck categories. Forum Math. 11, 735-759 (1999)

29. Colpi, R., Gregorio, E., Mantese, F.: On the heart of a faithful torsion theory. J. Algebra 307, 841-863 (2007)

30. Colpi, R., Trlifaj, J.: Tilting modules and tilting torsion theories. J. Algebra 178, 614-634 (1995)

31. Freyd, P.: Abelian categories: An introduction to the theory of functors. In: Harper's Series in Modern Mathematics, p. 164. Harper \& Row, New York (1964)

32. Franjou, V., Pirashvili, T.: Comparison of abelian categories recollements. Documenta Math. 9, 41-56 (2004)

33. Franke, J.: On the Brown representability theorem for triangulated categories. Topology 40, 667-680 (2001)

34. Gabriel, P., de la Peña, J.: Quotients of representation-finite algebras. Commun. Algebra 15(1-2), 279-307 (1987) 
35. Geigle, W., Lenzing, H.: Perpendicular categories with applications to representations and sheaves. J. Algebra 144(2), 273-343 (1991)

36. Gelfand, S., Manin, Y.: Methods of homological algebra. Springer Monographs in Mathematics, Springer, New York (2003)

37. Happel, D.: On the derived category of a finite dimensional algebra. Comment. Math. Helv. 62(3), 339-389 (1987)

38. Happel, D., Reiten, I., Smalø, S.O.: Tilting is abelian categories and quasitilted algebras. Mem. Am. Math. Soc. 120, viii+ 88 pp (1996)

39. Hogadi, A., Xu, C.: Products, homotopy limits and applications, preprint (2009). arXiv:0902.4016

40. Hoshino, M., Kato, Y., Miyachi, J.-I.: On t-structures and torsion theories induced by compact objects. J. Pure Appl. Algebra 167(1), 15-35 (2002)

41. Huybrechts, D.: Fourier-Mukai Transforms in Algebraic Geometry, Oxford Mathematical Monographs. Oxford University Press, Oxford (2006)

42. Illusie, L.: Complexe cotangent et déformations. I., Lecture Notes in Mathematics, vol. 239. SpringerVerlag, Berlin (1971)

43. Iyama, O., Jørgensen, P., Yang, D.: Intermediate co-t-structures, 2-term silting objects, tau-tilting modules, and torsion classes. Algebra Numb. Theory 8(10), 2413-2431 (2014)

44. Iyama, O., Kato, K., Miyachi, J.: Recollement of homotopy categories and Cohen-Macaulay modules. J. K-Theory 8, 507-542 (2011)

45. Jørgensen, P., Kato, K.: Triangulated subcategories of extensions, stable t-structures and triangles of recollements. J. Pure Appl. Algebra 219, 5500-5510 (2015)

46. Keller, B.: A remark on tilting theory and dg algebras. Manuscripta Math. 79, 247-252 (1993)

47. Keller, B.: Deriving DG categories. Ann. Sci. École Norm. Sup. (4) 27(1), 63-102 (1994)

48. Keller, B., Nicolás, P.: Weight structures and simple dg modules for positive dg algebras. Int. Math. Res. Not. 2013(5), 1028-1078 (2013). doi:10.1093/imrn/rns009

49. Keller, B., Vossieck, D.: Sous les catégories dérivées. C. R. Acad. Sci. Paris 305, 225-228 (1987)

50. Keller, B., Vossieck, D.: Aisles in derived categories. Bull. Soc. Math. Belg. Sér. A 40(2), 239-253 (1988)

51. Koenig, S., Yang, D.: Silting objects, simple-minded collections, t-structures and co-t-structures for finitedimensional algebras. Doc. Math. 19, 403-438 (2014)

52. Krause, H.: Localization theory for triangulated categories, triangulated categories, vol. 375, pp. 161-235, London Math. Soc. Lecture Note Ser. Cambridge Univ. Press, Cambridge (2010)

53. Krause, H.: Deriving Auslander's Formula. Documenta Math. 20, 669-688 (2015)

54. Krause, H., Le, J.: The Auslander-Reiten formula for complexes of modules. Adv. Math. 207(1), 133-148 (2006)

55. Krause, H., Stovicek, J.: The telescope conjecture for hereditary rings via Ext-orthogonal Pairs. Adv. Math. 225(5), 2341-2364 (2010)

56. Liu, Q., Vitória, J., Yang, D.: Glueing silting objects. Nagoya Math. J. 216, 117-151 (2014)

57. Miyachi, J.I.: Recollement and tilting complexes. J. Pure Appl. Algebra 183(1-3), 245-273 (2003)

58. Modoi, G.C.: The dual of Brown representability for some derived categories. Ark. Mat. 54(2), 485-498 (2016)

59. Mukai, S.: Duality between $D(X)$ and $D(\hat{X})$ and its aplications to Picard sheaves. Nagoya Math. J. 81, 153-175 (1981)

60. Murfet, D.: Derived categories Part I (2006). therisingsea.org

61. Neeman, A.: Some new axioms for triangulated categories. J. Algebra 139, 221-255 (1991)

62. Neeman, A.: Triangulated categories, Annals of Mathematics Studies, vol 148. Princeton University Press, Princeton (2001)

63. Neeman, A.: Non-left-complete derived categories. Math. Res. Lett. 18(5), 827-832 (2011)

64. Nicolás, P., Saorin, M.: Parametrizing recollement data for triangulated categories. J. Algebra 322, 12201250 (2009)

65. Nicolás, P., Saorin, M., Zvonareva, A.: Silting theory in triangulated categories with coproducts, preprint (2015). arXiv: 1512.04700

66. Orlov, D.: Derived categories of coherent sheaves and equivalences between them. Izv. Math. 66, 569-594 (2002)

67. Parra, C., Saorin, M.: Direct limits in the heart of a t-structure: the case of a torsion pair. J. Pure Appl. Algebra 219(9), 4117-4143 (2015)

68. Parshall, B., Scott, L.: Derived categories, quasi-hereditary algebras, and algebraic groups. Carlton Univ. Math. Notes 3, 1-111 (1989)

69. Psaroudakis, C.: Homological theory of recollements of abelian categories. J. Algebra 398, 63-110 (2014)

70. Psaroudakis, C., Vitória, J.: Recollements of module categories. Appl. Categ. Struct. 22(4), 579-593 (2014) 
71. Rickard, J.: Morita theory for derived categories. J. Lond. Math. Soc.(2) 39, 436-456 (1989)

72. Rickard, J.: Derived equivalences as derived functors. J. Lond. Math. Soc. (2) 43, 37-48 (1991)

73. Saneblidze, S.: On derived categories and derived functors. Extracta Math. 22(3), 315-324 (2007)

74. Schnürer, O.M.: Homotopy categories and idempotent completeness, weight structures and weight complex functors, preprint. arXiv:1107.1227

75. Schwede, S.: The stable homotopy category has a unique model at the prime 2. Adv. Math. 164, 24-40 (2001)

76. Stenström, B.: Rings of Quotients. Springer, New York (1975)

77. Štoviček, J.: Derived equivalences induced by big cotilting modules. Adv. Math. 263, 45-87 (2014)

78. Wei, J.: Semi-tilting complexes. Isr. J. Math. 194(2), 871-893 (2013)

79. Wildeshaus, J.: f-catégories, tours et motifs de Tate. C. R. Math. Acad. Sci. Paris 347(23-24), 1337-1342 (2009)

80. Yao, D.: On equivalences of derived categories. K-theory 10, 307-322 (1996)

81. Zimmermann, A.: Representation theory: a homological algebra point of view. Algebra Appl. Springer 19, $x x+707(2014)$ 